Claremont Colleges

Scholarship@ Claremont

HMC Senior Theses

HMC Student Scholarship

2013

\title{
A Comparison and Catalog of Intrinsic Tumor Growth Models
}

Elizabeth A. Sarapata

Harvey Mudd College

\section{Recommended Citation}

Sarapata, Elizabeth A., "A Comparison and Catalog of Intrinsic Tumor Growth Models" (2013). HMC Senior Theses. 52.

https://scholarship.claremont.edu/hmc_theses/52

This Open Access Senior Thesis is brought to you for free and open access by the HMC Student Scholarship at Scholarship @ Claremont. It has been accepted for inclusion in HMC Senior Theses by an authorized administrator of Scholarship @ Claremont. For more information, please contact scholarship@cuc.claremont.edu. 


\section{A Comparison and Catalog of Intrinsic Tumor Growth Models}

\section{Elizabeth Sarapata}

Lisette de Pillis, Advisor

Ami Radunskaya, Reader

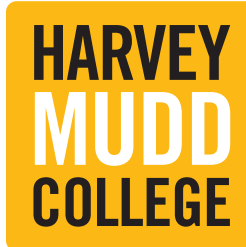

Department of Mathematics

May, 2013 


\section{Copyright () 2013 Elizabeth Sarapata.}

The author grants Harvey Mudd College and the Claremont Colleges Library the nonexclusive right to make this work available for noncommercial, educational purposes, provided that this copyright statement appears on the reproduced materials and notice is given that the copying is by permission of the author. To disseminate otherwise or to republish requires written permission from the author. 


\section{Abstract}

Determining the dynamics and parameter values that drive tumor growth is of great interest to mathematical modelers, experimentalists and practitioners alike. We provide a basis on which to estimate the growth dynamics of ten different tumors by fitting growth parameters to at least five sets of published experimental data per type of tumor. These timescale tumor growth data are also used to determine which of the most common tumor growth models (exponential, power law, logistic, Gompertz, or von Bertalanffy) provides the best fit for each type of tumor. In order to compute the best-fit parameters, we implemented a hybrid local-global least squares minimization algorithm based on a combination of Nelder-Mead simplex direct search and Monte Carlo Markov Chain methods. 



\section{Acknowledgments}

To Dr. Lisette de Pillis and Dr. Radunskaya, who pointed me in all the right directions. 



\section{Contents}

$\begin{array}{ll}\text { Abstract } & \text { iii }\end{array}$

Acknowledgments $\quad$ v

1 Introduction $\quad 1$

1.1 Introduction . . . . . . . . . . . . . . 1

1.2 Previous Research . . . . . . . . . . . . . . . 2

2 Assumptions and Methods $\quad 5$

2.1 Experimental data . . . . . . . . . . . . . 5

2.2 Unit normalization . . . . . . . . . . . . . 5

2.3 ODE Tumor Growth Models . . . . . . . . . . . . . . . . 6

2.4 Parameter fitting algorithms . . . . . . . . . . 7

2.5 Biologically Motivated Assumptions . . . . . . . . . . . . 11

2.6 Fitting evaluation metrics . . . . . . . . . . . . . . 12

2.7 Parameter Sensitivity Analysis . . . . . . . . . . . . . 12

3 Results $\quad 17$

3.1 Tumor Growth Parameter Values . . . . . . . . . . . . . . . . . 17

3.2 Parameter Sensitivity Analysis ... . . . . . . . . 22

4 Discussion $\quad 27$

4.1 Parameter Fitting . . . . . . . . . . . . . . . . 27

4.2 Parameter Fitting Algorithms . . . . . . . . . . . . . 28

4.3 Parameter Sensitivity Analysis . . . . . . . . . . . . . . 29

4.4 Usage of Least Squares Residuals versus BIC . . . . . . . . . 30

5 Future Work $\quad 33$

5.1 ODE models for CD47 Treatment . . . . . . . . . . . . 33 
viii Contents

A Supplemental Materials $\quad 37$

A.1 Sources of Data for Parameter Values . . . . . . . . . . . . . . 37

A.2 Results of Parameter Fittings . . . . . . . . . . . . . 40

$\begin{array}{ll}\text { Bibliography } & 81\end{array}$ 


\section{List of Figures}

3.1 Logistic Model of Control B16 Data from Agur 2011 . . . . . 23

3.2 Local Parameter Sensitivity Analysis for Five Models, Altering Parameters by $10 \% \ldots . . \ldots . . \ldots 24$

3.3 Local Parameter Sensitivity Analysis for Five Models, Altering Parameters by 10\% (with Power Law a results removed) 25

A.1 Parameter Fittings to In Vitro Bladder Cancer Trials _ . . . . 42

A.2 Parameter Fittings to Individual In Vivo Bladder Cancer Trials 43

A.3 Parameter Fitting to Combined In Vivo Bladder Cancer Trials 44

A.4 Parameter Fittings to Individual In Vitro Breast Cancer Trials 45

A.5 Parameter Fitting to Combined In Vitro Breast Cancer Trials 46

A.6 Parameter Fittings to Individual In Vivo Breast Cancer Trials 47

A.7 Parameter Fitting to Combined In Vivo Breast Cancer Trials . 48

A.8 Parameter Fittings to Individual In Vitro Colon Cancer Trials 49

A.9 Parameter Fitting to Combined In Vitro Colon Cancer Trials . 50

A.10 Parameter Fittings to Individual In Vivo Colon Cancer Trials 51

A.11 Parameter Fitting to Combined In Vivo Colon Cancer Trials . 52

A.12 Parameter Fittings to Individual In Vitro Head and Neck Squamous Cell Carcinoma Trials . . . . . . . . . . . . . 53

A.13 Parameter Fitting to Combined In Vitro Head and Neck Squamous Cell Carcinoma Trials . . . . . . . . . . . . . . . . 54

A.14 Parameter Fittings to Individual In Vivo Head and Neck Squamous Cell Carcinoma Trials . . . . . . . . . . . . . . 55

A.15 Parameter Fitting to Combined In Vivo Head and Neck Squamous Cell Carcinoma Trials . . . . . . . . . . . . . . 56

A.16 Parameter Fittings to Individual In Vitro Hepatocellular Carcinoma Trials . . . . . . . . . . . . . . . 57

A.17 Parameter Fitting to Combined In Vitro Hepatocellular Carcinoma Trials . . . . . . . . . . . . . . . . 58 
A.18 Parameter Fittings to Individual In Vivo Hepatocellular Carcinoma Trials . . . . . . . . . . . . . . . . . . 59

A.19 Parameter Fitting to Combined In Vivo Hepatocellular Carcinoma Trials . . . . . . . . . . . . . . . . . 60

A.20 Parameter Fittings to Individual In Vitro Lung Cancer Trials 61

A.21 Parameter Fitting to Combined In Vitro Lung Cancer Trials . 62

A.22 Parameter Fittings to Individual In Vivo Lung Cancer Trials . 63

A.23 Parameter Fitting to Combined In Vivo Lung Cancer Trials . 64

A.24 Parameter Fittings to Individual In Vitro Melanoma Trials . . 65

A.25 Parameter Fitting to Combined In Vitro Melanoma Trials . . 66

A.26 Parameter Fittings to Individual In Vivo Melanoma Trials . . 67

A.27 Parameter Fitting to Combined In Vivo Melanoma Trials . . . 68

A.28 Parameter Fittings to Individual In Vitro Ovarian Cancer Trials 69

A.29 Parameter Fitting to Combined In Vitro Ovarian Cancer Trials 70

A.30 Parameter Fittings to Individual In Vivo Ovarian Cancer Trials 71

A.31 Parameter Fitting to Combined In Vivo Ovarian Cancer Trials 72

A.32 Parameter Fittings to In Vitro Pancreatic Cancer Trials . . . . 73

A.33 Parameter Fittings to Individual In Vivo Pancreatic Cancer

Trials . . . . . . . . . . . . . . . . . 74

A.34 Parameter Fitting to Combined In Vivo Pancreatic Cancer Trials 75

A.35 Parameter Fittings to Individual In Vitro Renal Cell Carcinoma Trials . . . . . . . . . . . . . . . . . . 76

A.36 Parameter Fitting to Combined In Vitro Renal Cell Carcinoma Trials ....................... 77

A.37 Parameter Fittings to Individual In Vivo Renal Cell Carcinoma Trials . . . . . . . . . . . . . . . . 78

A.38 Parameter Fitting to Combined In Vivo Renal Cell Carcinoma Trials . . . . . . . . . . . . . . . . . 79 


\section{List of Tables}

3.1 Recommended Parameter Values and Ranges for Ten Different Types of Cancer and Five ODE Growth Laws . . . . . . . 19

3.2 Model Evaluation Metrics for Combined Experimental Data Fittings . . . . . . . . . . . . . . . 20

3.3 Model Fit Ranking According to Least Squares Residuals . . 21

3.4 Results of Partial Rank Correlation Coefficient Test for TwoParameter Growth Models . . . . . . . . . . . . . . . . . 26

A.1 Sources of Timescale Data by Type of Cancer and Cell Line . 37

A.1 Sources of Timescale Data by Type of Cancer and Cell Line . 38

A.1 Sources of Timescale Data by Type of Cancer and Cell Line . 39 



\section{Chapter 1}

\section{Introduction}

\subsection{Introduction}

Mathematical models of tumor-immune system interactions are an especially useful class of dynamical systems model, utilizing a vast range of mathematical methods to model stochastic biological processes. Many researchers choose to represent tumor-immune system interaction by nonlinear systems of differential equations that account for various interacting cell populations or concentrations of chemicals (Sanga et al., 2006; de Pillis et al., 2013a; Jackson and Byrne, 2000; Robertson-Tessi et al., 2012; Hart et al., 1998; de Pillis and Radunskaya, 2006). If pharmaceuticals are also taken into account, these models allow both for an unlimited number of patient trials and the ability to vary dosages and combinations of medications such that the likelihood of eliminating the tumor is maximized.

Although many facets of these models change-underlying assumptions, the specific cell populations being modeled, the functions used to approximate biological phenomena-they all must have a function that approximates tumor growth. Many researchers make this estimation by either citing an earlier paper or performing a fitting to one or two experimental data sets (Sanga et al., 2006; de Pillis et al., 2013a; Jackson and Byrne, 2000; Robertson-Tessi et al., 2012; Hart et al., 1998; de Pillis and Radunskaya, 2006). It may save a great deal of work and provide a stronger basis for tumor growth models to have an easily accessible catalog of tumor growth functions in one place, especially one that provides a range of possible values and uses a large number of different experimental data sources for the fittings. We have created such a catalog and encourage its use by mathematical researchers. Our catalog accounts for tumors originating from ten 
different organs, each with a minimum of seven data sets from a minimum of five different papers each.

In addition to improving the fit with a large amount of published experimental data, we also test five different tumor growth functions. There are five widely used growth functions in the field of dynamical systems tumor models: exponential growth functions, power law functions, logistic growth functions, Von Bertalanffy growth functions, and Gompertz growth functions (de Pillis and Radunskaya, 2006; Hart et al., 1998). For each of the ten types of tumor, we fit the experimental data to each of these growth functions and report several metrics to evaluate the fitting. This information will not only suggest which function is best suited to model the growth of each tumor type, but will provide an experimental basis for determining whether each individual function truly captures the dynamics of tumor growth as well.

With access to a large catalog of tumor growth data, we are uniquely able to implement a series of comparative models using CD47 treatment, a relatively new form of cancer treatment (Willingham, Stephen B. et al., 2012; B. Edris et al., 2012; Chen et al., 2005). Researchers have isolated a protein, CD47, which can be targeted on many different types of tumor for effective treatment. This is especially significant because cancer treatments are usually tailored according to the organ of origin and nature of the tumor. Current data on CD47 treatment record its effects on eight of the ten different types of tumor. We propose a preliminary model that can be used to explore tumor-specific dynamics with CD47 treatment. In future work we will take advantage of the data fittings we have made available, and using the high and low ranges of the tumor growth parameters with the best-fit tumor growth function we have determined, we will further develop and test our proposed CD47 treatment model.

\subsection{Previous Research}

Four of the different tumor growth functions have been studied side-byside by de Pillis for human melanoma grown in mice (de Pillis and Radunskaya, 2006). By a least-squares residual comparison, this study determined that Von Bertalanffy and logistic growth models provided the best fits for the experimental data. Only one set of experimental trials from one source was used to determine this result.

An earlier study by Hart et al. compared Gompertz, logistic, exponential and power growth law models in breast cancer (Hart et al., 1998). This 
study concluded that power law growth was the best candidate for the function governing the growth of breast cancer. However, in a later model developed in part by Zvia Agur, who worked on the previous paper, logistic growth instead of power law growth was used to represent the tumor growth equation (Elishmereni et al., 2011).

One of the earliest works comparing different tumor growth functions was published in 1973, and focuses on fitting tumor growth data to the Gompertz and logistic models (Aroesty et al., 1973). Rather than determining which growth function is the better fit to the data using fitting evaluation metrics, Aroesty's paper mentions the theoretical similarities between the Gompertz and logistic models. However, no direct comparisons of the fitting results are provided.

Similar studies have also been performed for comparing bacterial growth rates (López, S. et al., 2004). Lòpez 2004 used two data sets and nine different growth functions as opposed to four or five, and found that the most effective models for bacterial population growth were Baranyi, threephase-linear, Richards and Weibull growth models. The fact that no direct comparisons of the fitting results are made demonstrates that large-scale comparison projects of this character are a relatively recent ordeal. 



\section{Chapter 2}

\section{Assumptions and Methods}

\subsection{Experimental data}

We curated a database of timescale tumor growth data sets for bladder cancer, breast cancer, colon cancer, head and neck squamous cell carcinoma, hepatocellular carcinoma, lung cancer, melanoma, ovarian cancer, pancreatic cancer, and renal cell carcinoma. Each group of data sets was collected from at least five peer-reviewed publications, with the smallest-sized group containing seven data sets and the largest containing 17. In addition, at least one data set collected for each type of cancer was obtained from in vitro trials and at least one data set is composed of data collected from in vivo trials. Along with in vitro trials, the range of target organisms includes SCID mice, nude mice, normal mice, hamsters and humans. Table A.1 shows all sources for each timescale data set included in the study, as well as the cell lines for each trial.

\subsection{Unit normalization}

Of the papers which reported timescale tumor growth, the units and methods of measurement varied greatly. At least one paper per type of tumor was an in vitro trial that reported tumor size as a cell number, the preferred unit for our purposes, but all data from in vivo and in situ trials were presented in units of $\mathrm{mm}^{3}, \mathrm{~mm}^{2}, \mathrm{~mm}, \mathrm{~cm}^{3}$, or relative volume. In addition, instead of assuming a spherical tumor, volume was reported in a majority of papers as the product of the height, length and width of the tumor, overestimating the volume. However, we will also assume that no individual tumor cells are compressed, which will underestimate the number of tu- 
mor cells. The combination of these two assumptions is presumed to bring the estimated cell number within reasonable error of the real cell number.

In many cases, we were able to obtain an estimate of the number of tumor cells in a given volume from murine data sets which reported an initial cell count along with an initial volume measurement. We then divided the volume by the cell number, allowing for an estimate of the volume of a single tumor cell. We used this same estimate for data sets on tumor growth for tumors originating from the same organ. The most accurate conversion estimate, requiring the fewest conversions from the original data, was an estimate of $2.85 \times 10^{3}$ cells $/ \mu \mathrm{m}^{3}$ for pancreatic cancer (Kisfalvi et al., 2009). For types of tumor which did not have a conversion data set available, we estimated the conversion ratio at approximately $1.82 \times 10^{3}$ cells $/ \mu \mathrm{m}^{3}$ (de Pillis et al., 2013a). Although these two estimates were obtained from different sources and for different cells, it should be noted that they are the same order of magnitude despite the high variability of cell size.

This volume estimate of a tumor cell provides a method with which to convert volume, area or distance measurements to cell number. For those data sets which reported growth in volume, we normalized each datum by $\frac{1}{\mu_{T}}$ where $\mu_{T}$ is the tumor cell volume calculated as above. The papers that reported an area measurement all obtained the values by multiplying the minor axis of the tumor by the major axis (Ricker et al., 2004; Sunwoo et al., 2001; Boukerche et al., 1989; Juhl et al., 1997). In this case, we assumed a cubic tumor with a volume of $a \sqrt{a}$ where $a$ is the reported area measurement. This allows us to calculate cell number from volume as before. Another set of papers reported only the major axis of the tumor (Murgo, 1985; Burke et al., 1997; Fujimoto et al., 1995). Here, we assumed a spherical tumor with the radius being one-half the major axis, using the volume of the sphere to estimate the cell number. For those papers that reported relative volume, we converted the data to cell number using the information in the supplemental material sections of each paper (Okegawa et al., 2001; Fujiwara et al., 1993; Ahonen et al., 2000).

\subsection{ODE Tumor Growth Models}

We compare fittings of tumor data for five different ODE growth models; exponential, power law, logistic, Gompertz, and Von Bertalanffy. Let $P$ represent an arbitrary population and let $t$ represent time. Exponential growth 
models are the simplest ODE growth model, described by

$$
\frac{d P}{d t}=C P
$$

for some constant $C$. It is a special case of power law growth,

$$
\frac{d P}{d t}=C P^{a},
$$

where both $C$ and $a$ are parameters that must be fit to the data. Logistic growth, which incorporates a carrying capacity, is given by

$$
\frac{d P}{d t}=r P\left(1-\frac{P}{K}\right)
$$

where $r$ represents the intrinsic growth rate and $K$ represents the carrying capacity. Von Bertalanffy growth is the least complex growth model that incorporates a carrying capacity, given by

$$
\frac{d P}{d t}=r(K-P)
$$

The final commonly used tumor growth model is Gompertz growth,

$$
\frac{d P}{d t}=r \log \left(\frac{K}{P}\right) P .
$$

Of these models, we expect logistic growth to provide the best estimation of experimental data, since it approximates exponential growth at low populations while accounting for the resource-limited growth behavior at high populations. Unfortunately, very few data sets exist that allow tumors to grow large enough in order to get a proper estimate of the carrying capacity. We specifically sought out data sets that included data for large populations in order to properly compare the former two models with the latter three (Fujiwara et al., 1993; Takahashi et al., 1992; Murgo, 1985; Richmond et al., 1983; Kisfalvi et al., 2009; Reinmuth et al., 2002; Nakata et al., 1998; Caltagirone et al., 2000; Ricker et al., 2004).

\subsection{Parameter fitting algorithms}

All fitting results were obtained by minimizing the non-weighted least squares distance. We refer to the least-squares distance by the quantity $d$, where

$$
d(p)=\sqrt{\left(q_{1}-f\left(p, t_{1}\right)\right)^{2}+\cdots+\left(q_{n}-f\left(p, t_{n}\right)\right)^{2}}
$$


for each $t_{n}$ representing a time value at which data are collected, $q_{n}$ representing the experimentally determined data point at $t_{n}$ and $f(p, t)$ representing the fitting given parameter $p$ and time $t$. We say that $\phi$ is a best-fit parameter if $\phi$ minimizes $d(p)$.

The parameters for each tumor growth model were estimated using at least two least-squares distance minimization algorithms. For each ODE model, the ODE with parameters was solved numerically using MATLAB's ode 45 function, implementing a 4th and 5th order Runge-Kutta solver. We then minimized a least squares distance function between this solution and a target set of data using either MATLAB's built-in fminsearch function or a Markov chain fitting with simulated annealing. MATLAB's fminsearch is a Nelder-Mead simplex direct search function. Nelder-Mead is one of a class of local-search algorithms, which require that one choose a very good initial guess, or the method will not converge to a global minimum but instead iterate toward a nearby local minimum. The local minimum found may not produce the best fit (Lagarias et al., 1998). This necessitates the use of an alternate, global, data-fitting method. In the next sections we provide more detail describing both the local Nelder-Mead algorithm and a global minimization algorithm that implements a Markov chain fitting with simulated annealing.

\subsubsection{The Nelder-Mead simplex direct search}

The Nelder-Mead simplex direct search algorithm implemented in MATLAB's fminsearch and described in New Computing Environments: Microcomputers in Large-Scale Computing (Dennis and Woods, 1987), goes through $k$ iterations. It begins by sampling $n+1$ time values, which we will refer to as $x_{1}, x_{2}, \cdots, x_{n+1}$. These values are sorted in increasing order of their image under the target function $f(x)$, that is,

$$
f\left(x_{1}\right) \leq f\left(x_{2}\right) \leq \cdots \leq f\left(x_{n+1}\right) .
$$

The point with the $i^{\text {th }}$ lowest image at the $k^{\text {th }}$ iteration will be referred to as $x_{i}^{k}$. We then define a simplex $S_{k}$ with vertices $x_{1}, x_{2}, \cdots x_{n}$, denoted by $S_{k}=<x_{1}, x_{2}, \cdots, x_{n+1}>$. This simplex is then modified by reflection, expansion, contraction, or shrinkage, in that order, until a new simplex $S_{k+1}$ which converges more closely to the local minimum is determined. The first step reflects the largest vertex, $x_{n+1}$, through the centroid of the vertices $x_{1}, \cdots, x_{n}$. The new reflected vertex, $x_{r}$, is calculated by

$$
x_{r}=(1+\alpha) \bar{x}-\alpha x_{n+1}
$$


such that $\alpha=1$ and $\bar{x}$ is the centroid:

$$
\bar{x}=\frac{1}{n} \sum_{i=1}^{n} x_{i}
$$

If $f\left(x_{1}\right) \leq f\left(x_{r}\right)<f\left(x_{n}\right)$, we move to the next iteration of the search with $\left.S_{k+1}=<x_{1}, x_{2}, \cdots, x_{n}, x_{r}\right\rangle$. The fact that $x_{r}$ is not in order with the rest of the vertices is intentional. However, if $f\left(x_{r}\right)<f\left(x_{1}\right)$, implying that $f\left(x_{r}\right)$ is now the closest point to the local minimum, we continue the step by producing an expansion vertex

$$
x_{e}=\gamma x_{r}+(1-\gamma) \bar{x}
$$

where $\gamma=2$. We choose the expansion vertex if $f\left(x_{e}\right)<f\left(x_{1}\right)$ and the reflected vertex elsewise. If neither the reflected or expanded vertices are accepted, which corresponds to the case where $f\left(x_{n}\right) \leq f\left(x_{r}\right)$, we compute a contracted vertex. This contraction vertex is calculated as

$$
x_{c}=\beta x_{r^{\prime}}+(1-\beta) \bar{x}
$$

where $\beta=\frac{1}{2}$ and $x_{r^{\prime}}=\min \left(x_{n+1}, x_{r}\right)$. This new vertex becomes part of the new simplex if $f\left(x_{c}\right)<f\left(x_{n}\right)$, and a new iteration begins. However, if $f\left(x_{n}\right) \leq f(c)$, we move to shrink the simplex by replacing each $x_{i}$ with the midpoint of the line segment connecting $x_{1}$ and $x_{i}$, or

$$
x_{i(n e w)}=\frac{x_{1}+x_{i}}{2} .
$$

The new simplex $S_{k+1}$ is created by rearranging the new $x_{i}$ according to their images under $f(x)$. This algorithm produces a simplex that moves toward a local minimum and shrinks if it is unable to locate a deeper local minimum. Although there are several stopping criteria for this algorithm, MATLAB stops fminsearch when the distance traveled in parameter space during the last iteration is under a pre-set tolerance.

\subsubsection{Markov Chain fitting with Simulated Annealing}

We use a Markov chain Monte Carlo (MCMC) fitting with simulated annealing. The MCMC fitting is non-deterministic alternative and tests a wider range of values in parameter space than fminsearch, causing it to be more capable of locating sinks that contain global minima (Winkler, 2003; Gilks et al., 1996; Brooks et al., 2011). Markov chain fitting itself starts by 
choosing an initial condition, a point in parameter space, denoted by $\mathbf{p}_{0}$. On the $i^{\text {th }}$ iteration of the fitting, a vector $\mathbf{v}$ of random magnitude and direction is determined from a set of uniformly distributed values over a closed disk of radius $r$, generating a new set of parameters $\mathbf{p}_{\text {new }}=\mathbf{p}_{i}+\mathbf{v}$. For a minimization function $d^{\prime}(p)$ (usually the least-squares distance,) we determine the output of this step by comparing $d^{\prime}\left(p_{i}\right)$ and $d^{\prime}\left(p_{\text {new }}\right)$. If $d^{\prime}\left(p_{\text {new }}\right) \leq d^{\prime}\left(p_{i}\right)$, we let $\mathbf{p}_{n+1}=\mathbf{p}_{\text {new }}$ with probability 1 . However, if $d^{\prime}\left(p_{\text {new }}\right)>d^{\prime}\left(p_{i}\right)$, i.e., the least-squares distance is smaller with the older parameters, we move to the new parameters with probability

$$
\frac{d^{\prime}\left(\mathbf{p}_{i}\right)}{d^{\prime}\left(\mathbf{p}_{\text {new }}\right)}
$$

Although this method seems counterintuitive, the action of moving away from local minima ensures that the Markov chain fitting function is more likely to locate global minima. This serves two purposes-to escape more easily from a local minimum that is not a global minimum, and to allow repeated iterations of the same algorithm to increase the accuracy of the results. The stochasticity in the algorithm allows for different outcomes even among trials with the same initial conditions (Winkler, 2003; Gilks et al., 1996; Brooks et al., 2011). This process is repeated $n$ times- where $n$ is an arbitrary number chosen by the user-before stopping. This algorithm has no standard stopping condition. In our case, we chose $n=200$. This number of runs allowed us to achieve relatively good fits while keeping computational running times reasonable. Implementing this algorithm with a larger $n$ would increase the chance that a global minimum is located.

Simulated annealing is the process of fitting not to the distance function, but the distance function raised to successive powers from 0 to 1 , where the result of each fitting is used as the initial condition for the next fitting. This algorithm reduces the chances that a minimization function will converge to a local minimum instead of a global minimum, since the act of raising the distance function to a power less than 1 reduces the prominence of local minima (Winkler, 2003). In this case, we ran 10 trials with simulated annealing, corresponding to ten iterations. The $n^{\text {th }}$ iteration uses the Markov chain fitting algorithm to minimize the function $(d(p))^{\beta_{n}}$ for $\beta_{n}=0.1 n$. The fitting for $\beta_{n}=1$, i.e., the parameter values for $n=10$, are accepted as the final parameters for this iteration of the fitting. 


\subsubsection{Hybrid Minimization Algorithm}

Note that part of the difference in usage and performance between these two algorithms arises from the fact that Nelder-Mead simplex direct search, i.e. fminsearch, finds local function-minimizing parameters, but MCMC and Markov chain methods are more likely to find global function-minimizing parameters (Ashyraliyev et al., 2009). That is, while fminsearch will return the parameter values that produce the lowest least-squares fitting within a bounded neighborhood of the initial parameters, all Markov chain methods return the parameter values that produce the lowest leastsquares fitting over a finite number of arbitrary parameters from anywhere in the parameter space (Ashyraliyev et al., 2009). This difference in the domain of each algorithm leads to defining behaviors that either help or hinder the goodness of fit. Since fminsearch is a local minimizer of the least squares distance, it is excellent at converging to local minima, but is known to ignore global minima that may produce a better fit. Likewise, MCMC and Markov chain methods have the ability to locate other minima that may be far removed from the initial conditions, but are less likely to hone in on the exact minimum in a local sink.

In order to address the respective shortcomings of global and local parameter fitting algorithms, we used a hybrid of Nelder-Mead simplex direct search and Markov chain fitting with simulated annealing. We started with one round of fminsearch fitting, and the resulting parameters were passed as initial conditions to the Markov chain algorithm. If the resulting parameters did not meet visual standards or had singularities, the parameters underwent another round of Markov chain fitting. Next, since Markov chain fitting is effective at breaking out of local minima but less effective at converging to minima, a second round of fminsearch was performed using the results of the Markov chain fitting as initial conditions to ensure convergence to the deepest local minimum. All parameters reported in the Results section were determined by this sequence of fitting algorithms.

\subsection{Biologically Motivated Assumptions}

To determine the recommended parameters for each model, we recorded the parameters of the function that best represented all trials with the same model organism at once. However, in order to determine the full range of parameters, we performed fittings to each data set individually and recorded the extrema of each set of parameters. It is also assumed that 
in vitro trials are better indicators of intrinsic tumor growth rates, due to the lack of an immune system in the growth environment; and that in vivo trials are better indicators of animal carrying capacity, since the growth media are closer to conditions the tumor would encounter in a living organism. Thus, when relevant, intrinsic growth rates are determined from in vitro trials only and carrying capacities are determined from in vivo trials only. In cases where no carrying capacity is given, i.e., the exponential and power law growth models, only in vitro trials are used to determine the growth rate, and the in vitro trials are also used to determine the exponent for the power law model.

\subsection{Fitting evaluation metrics}

We can compare the goodness-of-fit between the output of fminsearch and MCMC by noting the least-squares residuals for each data set. Using this metric, lower residuals will suggest a better fitting. We also use the leastsquares residuals to calculate the Bayesian Information Criterion (BIC) for each fitting, which accounts for goodness-of-fit as well as the number of parameters to guard against over-fitting. The particular formula that we use is

$$
B I C=n \cdot \ln \left(\frac{1}{n-1} d(p)\right)+k \cdot \ln (n) .
$$

where $x$ represents the experimental data, $n$ is the number of data points in $x$, and $k$ is the number of parameters which are being estimated in the model (Priestley, 1981). For our purposes, $k=1$ for the exponential model and $k=2$ for the four other models. Note the inclusion of the leastsquares residuals. The primary reason for using BIC in conjunction with least-squares residuals in model evaluation is to determine the effects of using more parameters than necessary. The Bayesian Information Criterion incorporates slightly more information than the least squares residuals, penalizing models for using a larger number of parameters.

\subsection{Parameter Sensitivity Analysis}

We use two separate parameter sensitivity analysis techniques: a localized parameter sensitivity algorithm, and the Partial Rank Correlation Coefficient (PRCC) test using Latin Hypercube Sampling (LHS). These techniques account for the effects of slight parameter modifications on the overall function and accuracy of the model. 
The local parameter sensitivity analysis is used to determine how altering the value of a single parameter affects the behavior of the overall model Mummert (2010). In order to perform a local parameter sensitivity analysis, we start with an initial set of parameters $\mathbf{p}$, then choose a percentage $0<p<1$, an initial condition $y_{0}$ for the ODE model, and a time $t>0$. Then, where $p_{j}$ represents the $j^{\text {th }}$ parameter, we let $y_{t}$ be the value at $t$ of the ODE model with parameters $\mathbf{p}$ starting at $y_{0}$, let $y_{+}$be the value at $t$ of the ODE model with parameters $\left(p_{1}, \cdots, p_{j}(1+p), \cdots, p_{n}\right)$ starting at $y_{0}$, and let $y_{-}$be the value at $t$ of the ODE model with parameters $\left(p_{1}, \cdots, p_{j}(1-p), \cdots, p_{n}\right)$ starting at $y_{0}$. We then calculate the percentages of change, $\mathrm{C}_{\%+}$ and $\mathrm{C}_{\%_{-}}$, from the previous model output to the new model output as

$$
\begin{gathered}
C_{\%+}=\frac{y_{+}-y_{t}}{y_{t}} \times 100, \\
C_{\%-}=\frac{y_{-}-y_{t}}{y_{t}} \times 100 .
\end{gathered}
$$

If the model value and the value of an individual parameter are positively correlated, we expect $C_{\%+}$ to be positive and $C_{\%-}$ to be negative. Similarly, if the model value and the value of an individual parameter are negatively correlated, we expect $C_{\%+}$ to be negative and $C_{\%-}$ to be positive. In addition, a larger $\mathrm{C}_{\%+}$ or $\mathrm{C}_{\%-}$ - value indicates that the parameter $p_{j}$ has a more significant effect on the behavior of the model.

One of the issues with only using local parameter sensitivity analysis is that it does not account for interactions between parameters. This is why we use a second parameter sensitivity analysis technique, the Partial Rank Correlation Coefficient (PRCC). We begin by using Latin Hypercube Sampling (LHS) to generate a sampling of random vectors in parameter space. Contrast this with the local parameter sensitivity analysis, which can only evaluate the model along the axes of parameter space. For some integer $N>0$, LHS attempts to cover parameter space by separating a bounded subset of parameter space into $N$ sections and choosing a random value in each section from a uniform distribution Mummert (2010). MATLAB has a built-in function for performing LHS: Ihsdesign. This function returns a matrix of parameter values between 0 and 1 . Since these values are not viable to use as carrying capacities, we multiplied each set of carrying capacity parameters by $10^{9}$. We let $M$ be the matrix returned by lhsdesign.

From the results of the LHS, we generate an output vector, $\mathbf{y}$, where $y_{j}$ is the model value at time $t$ using the parameters given by the $j^{\text {th }}$ row 
of $M$. The first step to implementing the PRCC test is to rank transform the matrices, which takes $M$ and $\mathbf{y}$ as input and returns a matrix of the same dimensions, called $\underline{M}$ and $\mathbf{y}$, where each column contains all of the integer values from 1 to $N$. The ordering of these integers corresponds to the ordering of values in the original matrices, where a 1 in the $k^{\text {th }}$ column of $\underline{M}$ corresponds to the position of the lowest value in the $k^{\text {th }}$ column of $M$ and $N$ corresponds to the highest value.

We then use the rank transformed matrices to calculate the linear regression models for each parameter, $\overline{p_{k}}$, which expresses the target parameter $p_{k}$ as a linear combination of all of the other parameters. The equation

$$
\overline{p_{k}}=c_{0}+c_{1} p_{1}+\cdots+c_{k-1} p_{k-1}+c_{k+1} p_{k+1}+\cdots c_{n} p_{n}
$$

is solved by

$\underline{c}=\left(X^{T} X\right)^{-1}\left(X^{T} \underline{p_{k}}\right) ; \quad \underline{c}=\left[\begin{array}{c}c_{0} \\ \vdots \\ c_{n}\end{array}\right]$ and $X=\left[1, p_{1}, \cdots p_{k-1}, p_{k+1}, \cdots p_{n}\right]$.

In a similar manner, we define

$$
\overline{y_{p_{k}}}=a_{0}+a_{1} p_{1}+\cdots+a_{k-1} p_{k-1}+a_{k+1} p_{k+1}+\cdots a_{n} p_{n}
$$

which is solved by

$\underline{a}=\left(X^{T} X\right)^{-1}\left(X^{T} \underline{y}\right) ; \quad \underline{a}=\left[\begin{array}{c}a_{0} \\ \vdots \\ a_{n}\end{array}\right]$ and $X=\left[1, p_{1}, \cdots p_{k-1}, p_{k+1}, \cdots p_{n}\right]$.

This allows us to define two sets of residuals; $\operatorname{res}\left(p_{k}\right)=p_{k}-\overline{p_{k}}$ and $\operatorname{res}\left(y_{p_{k}}\right)=$ $y-\overline{y_{p_{k}}}$. We finalize the PRCC algorithm using the MATLAB function corrcoef to determine the correlation coefficient between $\operatorname{res}\left(p_{k}\right)$ and $\operatorname{res}\left(y_{p_{k}}\right)$. These correlation coefficients are the final result of the PRCC test, and they give a measure for the strength of the relationship between two parameters. A correlation coefficient of 1 between two parameters implies a strong positive linear relationship between the parameters, while a correlation coefficient of -1 between two parameters suggests a strong negative relationship. 
If the correlation coefficient is between -0.5 and 0.5 , the parameters do not have a linear relationship.

It should also be noted that PRCC can only be used on models that have a monotonic relationship on the model output, which is true of all parameters in our case, and the model must have two or more parameters in order to perform the test. This excludes the exponential model from PRCC analysis, as it only needs one parameter. 



\section{Chapter 3}

\section{Results}

\subsection{Tumor Growth Parameter Values}

In order to determine a set of recommended parameters and appropriate range for each type of cancer and growth model, we fit the parameters of each growth equation to a minimum of five data sets per type of cancer. These parameters fall into three different classes: intrinsic growth rates (denoted $r$ ), exponents (denoted $a$ ) and carrying capacities (denoted $K$.) It is assumed that intrinsic growth rates and exponents could be determined more accurately from in vitro and SCID mouse trials, i.e., due to the lack of an immune system interfering with growth; and that carrying capacities for humans are closer to the carrying capacities from in vivo trials, given that carrying capacities are highly dependent on the organ of origin belonging to each type of cancer. Therefore, for all models except the Power Law model, the intrinsic growth rate is determined from in vitro trials and the carrying capacity, if it exists, is determined from in vivo trials only. The Power Law is a special case which is addressed in the discussion section.

To fit the parameters, we used a hybrid of Monte Carlo fitting with simulated annealing and Nelder-Mead simplex direct search. Nelder-Mead, in the form of MATLAB's fminsearch function, was used to initialize parameters for the Monte Carlo fitting, which was performed to nudge the results of fminsearch out of local minima, then a second round of Nelder-Mead is used to lower the least squares residuals to the closest local minimum. In addition, two different types of fittings were performed on each set of related data sets. The in vitro trials for each type of cancer were fitted separately for the best fit parameters to determine an acceptable parameter range, then together with different initial conditions to determine the sug- 
gested parameter values.

As a result of the parameter fitting, a catalog of suggested values and ranges was found for ten types of cancer and five models, in Table 2.1. The least squares residuals and BIC values for the combined fittings can be found in Table 2.2. In order to highlight the best fittings and the relationship between least squares residuals and BIC values, the lowest least squares residuals values and BIC values in each row have been highlighted in purple and blue, respectively. Graphs for each individual fitting and combined fittings, as well as the residuals, parameters and sources for all fittings, can be found in the Supplemental Data section. We were also able to determine a ranking of model fit for each cancer type from the evaluation metrics, displayed in Table 2.3. This ranking was determined by comparing the sum of the least squares residuals for all individual and combined trials for each type of cancer. 


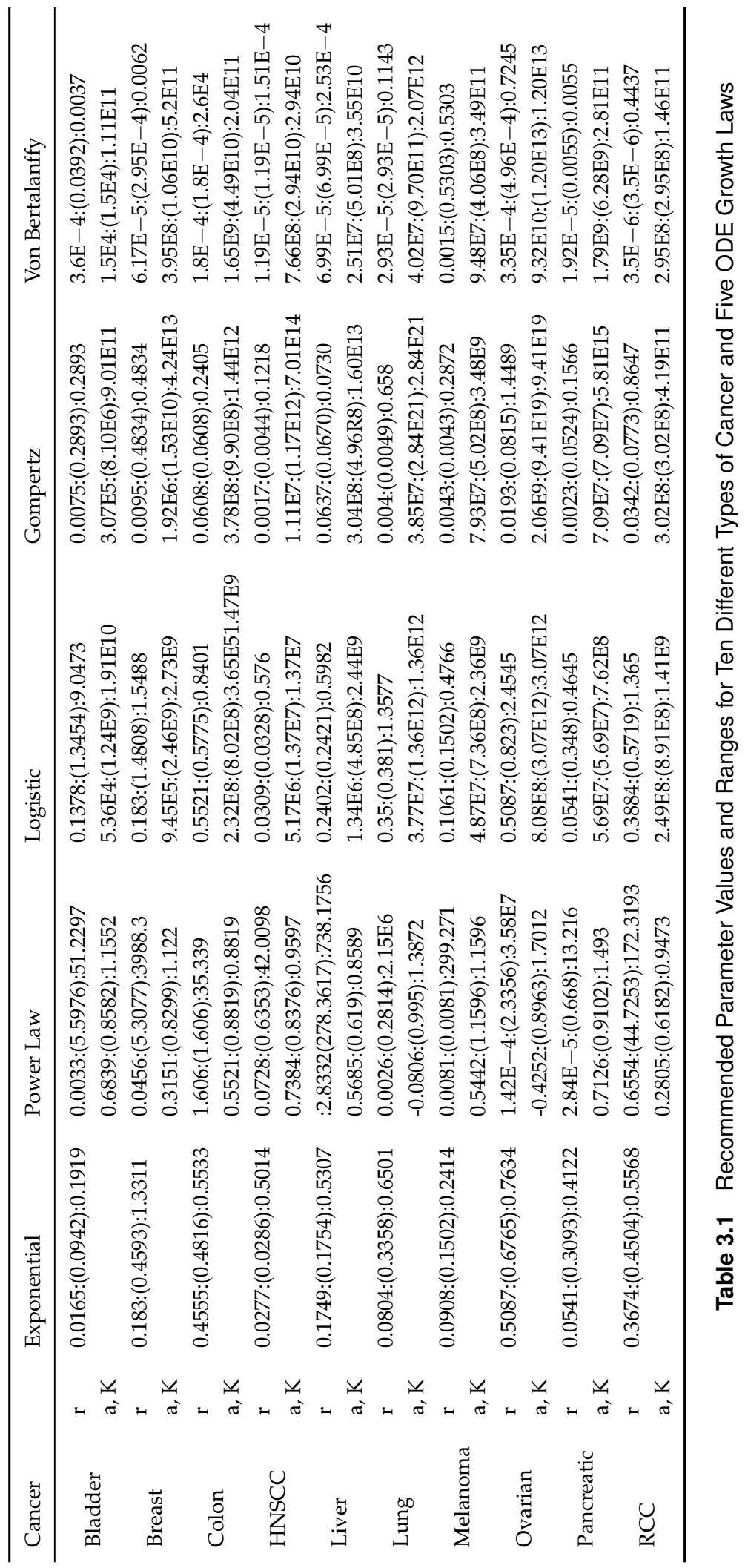




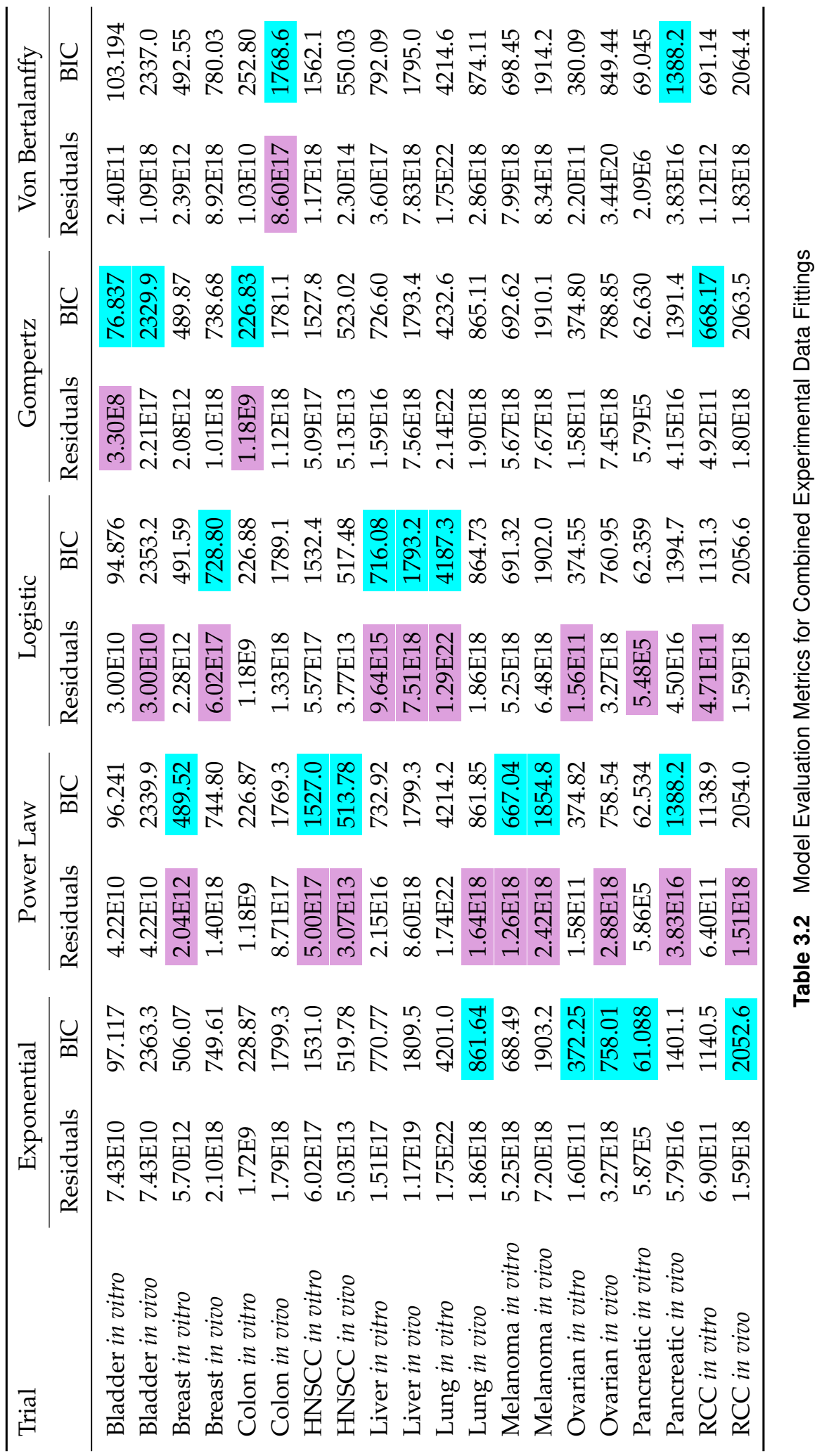




\begin{tabular}{lccccc}
\hline Cancer & \multicolumn{5}{c}{ Model Ranking } \\
& 1 & 2 & 3 & 4 & 5 \\
\hline Bladder & Power Law & Gompertz & Logistic & Exponential & Von Bertalanffy \\
Breast & Logistic & Gompertz & Power Law & Exponential & Von Bertalanffy \\
Colon & Power Law & Von Bertalanffy & Gompertz & Logistic & Exponential \\
HNSCC & Gompertz & Power Law & Exponential & Logistic & Von Bertalanffy \\
Liver & Logistic & Gompertz & Power Law & Von Bertalanffy & Exponential \\
Lung & Logistic & Power Law & Gompertz & Von Bertalanffy & Exponential \\
Melanoma & Power Law & Logistic & Exponential & Gompertz & Von Bertalanffy \\
Ovarian & Power Law & Exponential & Gompertz & Logistic & Von Bertalanffy \\
Pancreatic & Power Law & Gompertz & Logistic & Exponential & Von Bertalanffy \\
RCC & Power Law & Logistic & Exponential & Gompertz & Von Bertalanffy
\end{tabular}

Table 3.3 Model Fit Ranking According to Least Squares Residuals

In addition to building a new catalogue, we have tested our methods on established results as well. Agur et al. 2011 estimates logistic tumor growth parameters from expermiental data regarding B16 growth, some of which is reported (Elishmereni et al., 2011). When applied to the given control B16 data in Agur 2011, our parameter fitting algorithm returned an intrinsic growth rate of $r=0.5392$ and a carrying capacity of 7.4944E8 with least squares residuals of $8.60 E 14$ for the logistic fit. Agur et al.'s original result was $r=0.0014$ and 1.5E9 (Elishmereni et al., 2011). As their leastsquares residuals and exact data sets used for parameter estimation were not reported, we were unable to perform a suitable comparison to their parameters. 


\subsection{Parameter Sensitivity Analysis}

Two types of parameter sensitivity analysis were performed on the individual tumor growth models. The local parameter sensitivity analysis is performed to measure what the effect on the model would be if a parameter were increased or decreased by some percentage of its value, while the Partial Rank Correlation Coefficient test, commonly referred to as the PRCC, is intended to measure the statistical influence on the model output of parameters that have monotonic but nonlinear behavior Gomero (2012). As it is impossible to determine PRCC values from a model that has only one parameter, the exponential model is excluded from PRCC analysis.

We perform a local parameter sensitivity analysis altering each parameter by $10 \%$, with an initial condition of $1 \times 10^{4}$ tumor cells, running the model for 10 days, and starting with the parameters from the individually determined in vitro colon trials. The results are presented in Figure 3.2 and Figure 3.3 (where Figure 3.3 has the power law exponent removed to increase readability of the percent changes associated with the other parameters.) We also provide a PRCC analysis over 1000 randomized parameter values using Latin hypercube sampling, which is presented in Table 3.4. 


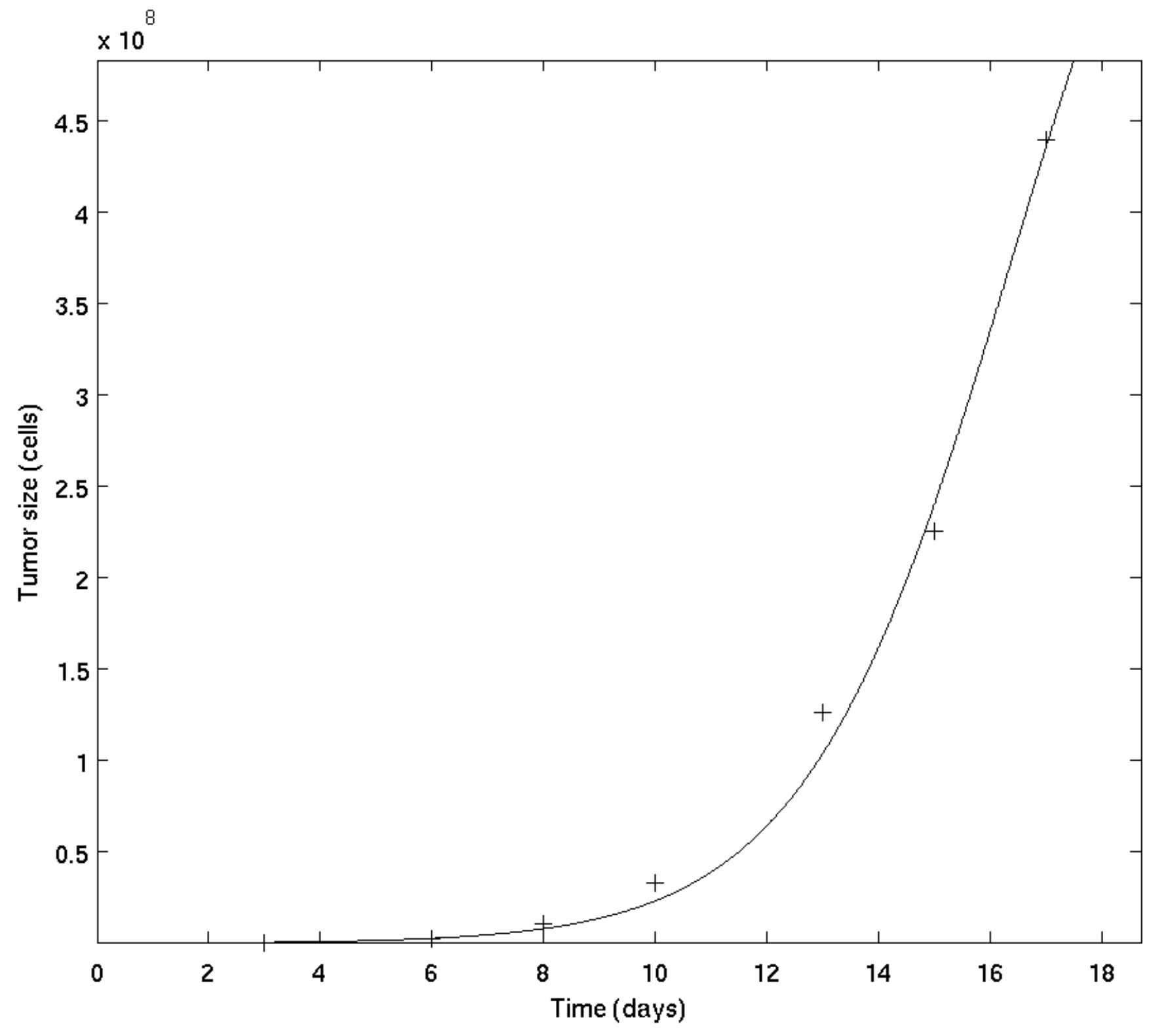

Figure 3.1 Logistic Model of Control B16 Data from Agur 2011 


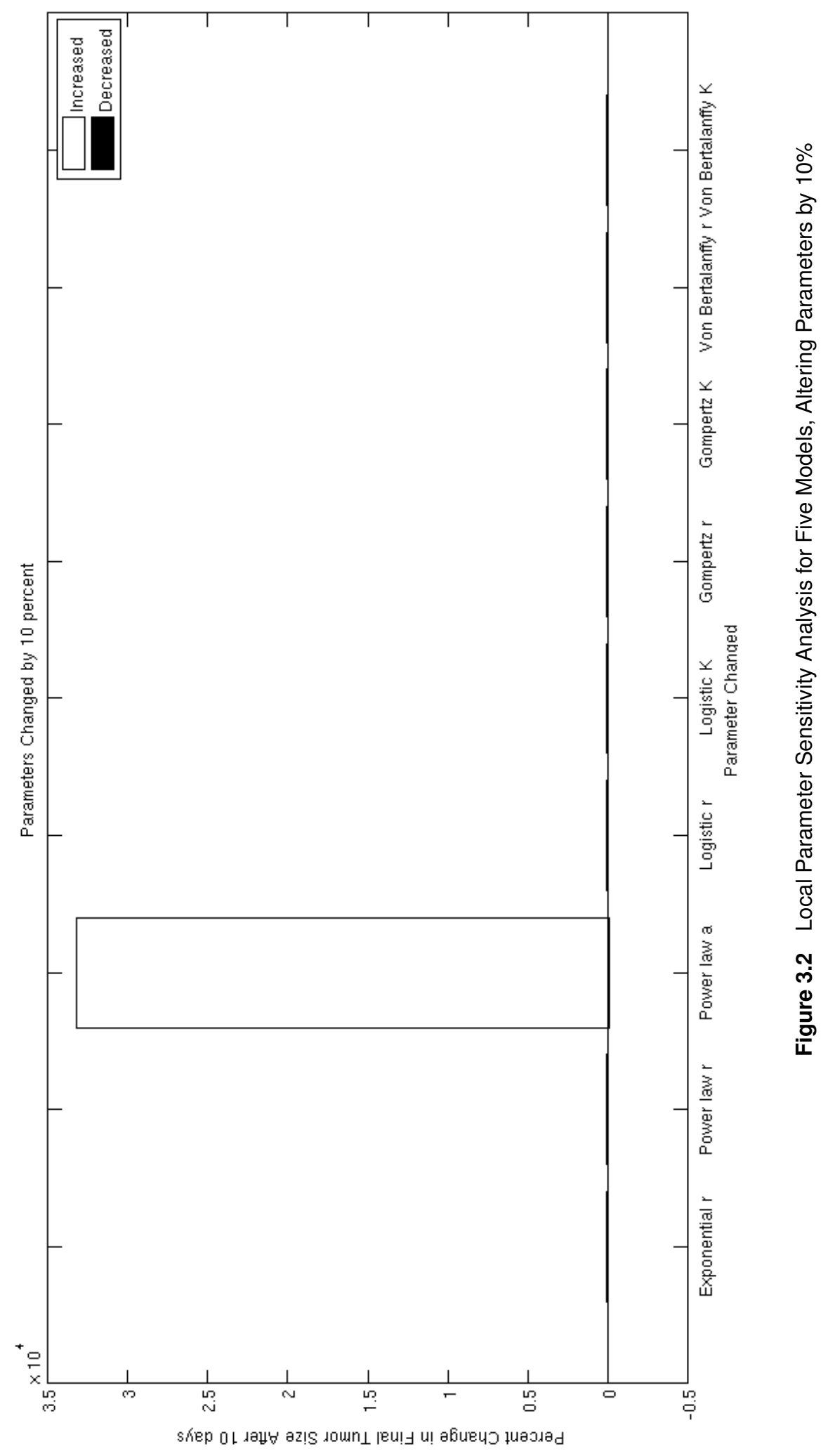




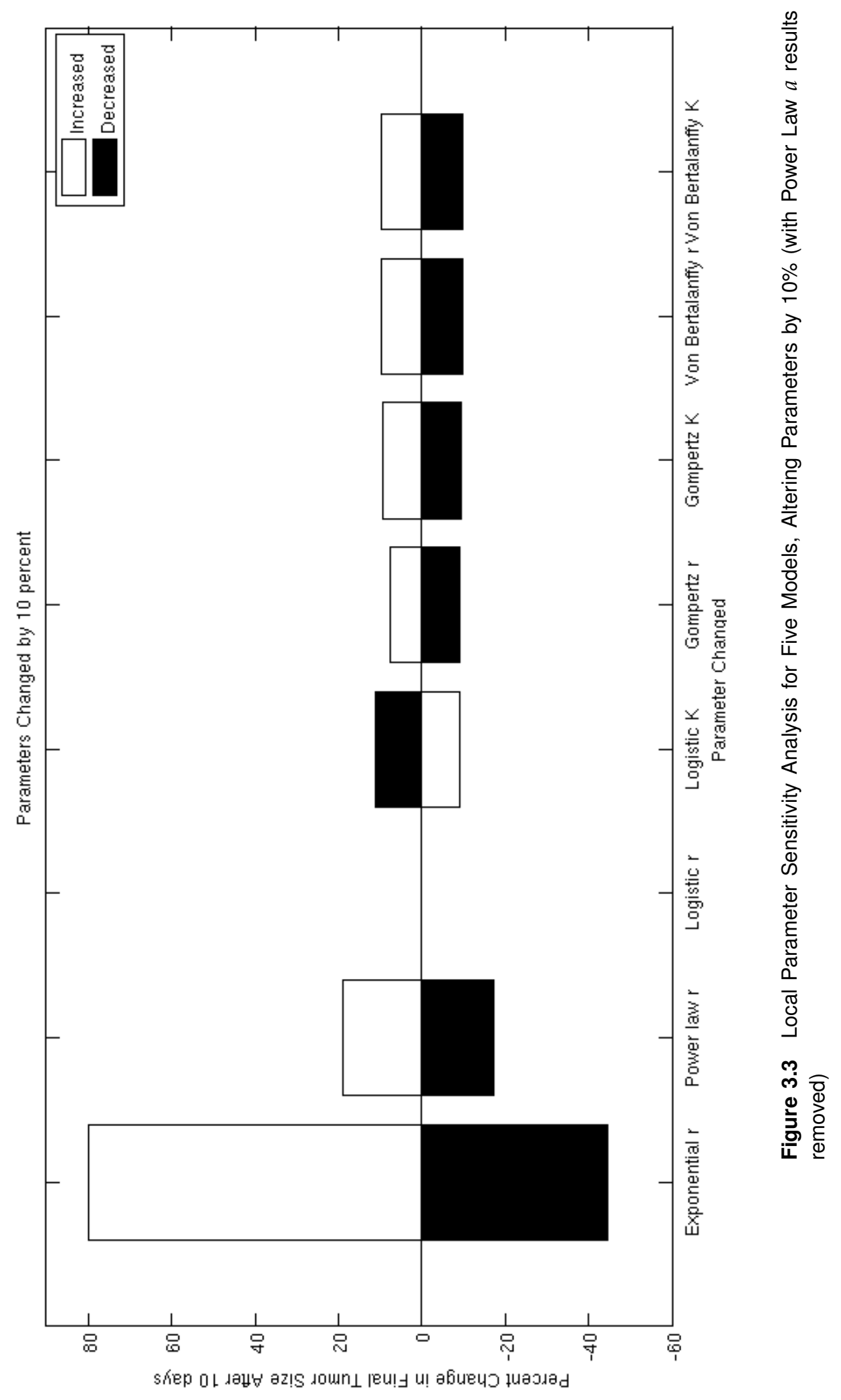




\begin{tabular}{cc}
\hline Parameter & PRCC \\
\hline Power law $r$ & 0.0412 \\
Power law $a$ & 0 \\
Logistic $r$ & 0 \\
Logistic $K$ & 0 \\
Gompertz $K$ & 0.0292 \\
Gompertz $K$ & 0 \\
Von Bertalanffy $K$ & -0.0104 \\
Von Bertalanffy $K$ & 0
\end{tabular}

Table 3.4 Results of Partial Rank Correlation Coefficient Test for TwoParameter Growth Models 


\section{Chapter 4}

\section{Discussion}

\subsection{Parameter Fitting}

In some cases, the results of the fitting algorithm are misleading. Logistic growth fittings sometimes ended with a carrying capacity with an order of magnitude much higher than comparable trials and the same intrinsic growth rate as the exponential fit to the same data. This occurs with in vivo trial 3 for breast cancer, in vivo trial 4 for head and neck squamous cell carcinoma; in vitro trials 1,2, and 10 and in vivo trial 1 and the combined in vivo fit for lung cancer; and in vitro trials 1 and 3 for ovarian cancer. When this happens, we assume that the exponential fit is a better match to the data than the logistic fit, so the logistic growth function approximates exponential growth by raising the carrying capacity to a number high enough so that it does not affect the fitting. This theory is supported by the least squares residuals; the least squares residuals from the exponential fit and the residuals from the logistic fit are the same when this situation occurs.

Another result determined from the parameter fitting process is that it may not be justifiable to alter power law growth parameters, even within the range given by repeated fits. This is because the best fit power law parameters occasionally have uncharacteristically high intrinsic growth rates (e.g. in vivo breast cancer trials 1 and 2, the combined head and neck squamous cell carcinoma in vivo trial, in vitro lung trial 5) and exponents that are lower than the exponents in trials in the same cancer. These results suggest that power law fitting is highly sensitive, where the intrinsic growth rates rise unpredictably to accommodate lower exponents and vice versa. Therefore, although power law fits occasionally have lower residuals than the other growth laws, their unstable nature prevents researchers from being 
able to justifiably change parameters within a certain range. For this reason, we discourage future tumor growth modelers from using the power law model.

One concern that must be addressed is whether the best-fit parameters are biologically accurate (Slezak et al., 2010). We note that the best-fit Von Bertalanffy parameters, which are expected to have intrinsic growth rates similar to all other models, consistently have intrinsic growth rates that are two or three orders of magnitude smaller. This is enough of an indication to doubt the biological veracity of the Von Bertalanffy parameters obtained by least-squares fitting. In addition, we have reason to question the biological relevance of the power law fittings for the same reasons as previously addressed.

\subsection{Parameter Fitting Algorithms}

To see that the hybrid fitting algorithm is more effective than either the Nelder-Mead simplex direct search or Markov Chain method with simulated annealing, we note that a set of parameters is only accepted if the least squares residuals are lower than they were in the previous fitting. Since fminsearch is used to provide initial values for the Markov Chain method, the residuals of a Nelder-Mead simplex direct search on a given data set bound the residuals of the hybrid search from above. Due to the nondeterministic nature of the Markov Chain method, the residuals are not necessarily always greater than those of the hybrid method, but it is true that the residuals returned by a specific iteration of the Markov Chain method will always be greater than the results of the hybrid algorithm using that specific iteration of the Markov Chain method. We have noted the inability of the Markov chain method to converge on global minima.

What is questionable, though, is the justification of using a much more complicated fitting algorithm than is necessary when fminsearch would have been sufficient. One issue with fminsearch is the inability to converge to a better minimum once a local minimum is detected by the algorithm, and to improve the fitting would necessitate changing parameters by hand. Since this project required 20 separate parameter fitting trials each to 70 data sets, not including the 90 combined fittings, manually altering parameters was not a viable option. Thus, even one instance of fminsearch converging to a non-global minimum would necessitate the use of a stronger parameter fitting algorithm. This hybrid method was adopted after repeated difficulties with fminsearch which would have remained unfixable otherwise.

As a side note, it is possible to fit the equations with carrying capacities 
in two different ways: the first, defining the parameters to be estimated as $r$ and $K$, and the second, defining the parameters to be estimated as $r$ and $1 / K$. Although theoretically equivalent, these two approaches can produce different outcomes depending on which fitting metric is used. For the logistic equation defined as

$$
\frac{d P}{d t}=r P\left(1-\frac{P}{K}\right)
$$

it is possible that the fitting algorithm may be slower in converging to the best-fit $K$, because it is possible for the best-fit $K$ to be several orders of magnitude higher than the initial condition. However, for the logistic equation defined as

$$
\frac{d P}{d t}=r P(1-b P)
$$

where $b=\frac{1}{K}$, both the Nelder-Mead simplex direct search and the Markov Chain method occasionally produced results where the carrying capacity was negative. This is a result of the relative distance in parameter space from the negative real axis; $1 \times 10^{6}$ and $-1 \times 10^{6}$ are much further apart than $1 \times 10^{-6}$ and $-1 \times 10^{-6}$, for example, thus the first condition makes it more difficult for either algorithm to reach negative values. We recommend using the first fitting method in order to avoid the fittings from producing a biologically inaccurate carrying capacity.

\subsection{Parameter Sensitivity Analysis}

While it may seem odd to perform a sensitivity analysis on a series of models that each have only one or two parameters, these techniques can be interpreted to compare the justifiability of tweaking parameters in each growth model. The PRCC values provide a measure of the strength of the relationship between two parameters, while the local parameter sensitivity analysis measures the effect of individual parameters on the model output. Therefore, while the local parameter sensitivity analysis can be used to estimate the effects of changing the value of a single parameter, the PRCC measure can tell us whether altering a single parameter while leaving the other constant is justifiable. If the local parameter sensitivity analysis reveals that altering a parameter by a small amount changes the output of the model by a significant amount, possibly $50 \%$ or greater, researchers should be warned against changing these parameters. Furthermore, if the PRCC 
indicates that two parameters have a low correlation coefficient, it indicates that the parameters have a non-monotonic relationship Gomero (2012).

For our purposes, the sensitivity analyses can be used to provide a basis for our claim that the power law is not a viable model. Figure 3.2 suggests that $a$, the exponential component of the power law model, affects the model output at a much higher percentage than any other parameter in any other model. In fact, increasing $a$ by only $10 \%$ caused the tumor to grow almost $35000 \%$ larger in only 10 days. This suggests that altering $a$ individually would change the tumor growth behavior at a massive rate that has no biological justification. An alternative would be to alter $a$ and $r$ in conjunction, such that the relatively low least squares residuals for the fitting are preserved. However, as the PRCC results suggest, the relationship between $a$ and $r$ is highly nonlinear. This is not suggestive in and of itself-none of the other parameters had significant PRCC resultsrather, we draw the conclusion in light of the results of the local parameter sensitivity analysis. In practice, a researcher seeking to lower the growth rate or raise the exponent of some of the less biologically sound power law fittings would have difficulty determining a relationship between $a$ and $r$ which allows the parameters to be altered while preserving the behavior of the original curve. This rigidity and extreme sensitivity is what makes the power law a less than ideal choice for a tumor growth model.

It should be noted in the local parameter sensitivity analysis that changing the logistic $r$ appears to have no effect on parameter sensitivity. This occurs because with the chosen parameters, the model reaches its carrying capacity by $t=10$ for all values in the sampled range of $r$, and a different percent growth would be obtained if the tumor growth were sampled at a different time.

\subsection{Usage of Least Squares Residuals versus BIC}

Although only using one model evaluation metric would not be thorough, it was necessary for the purpose of this project to choose one evaluation metric as the dominant one. The model performance ended up being ranked primarily by least squares residuals. Although the BIC can be seen as an extension of the least squares residual and incorporates more information, it was used as only a secondary evaluative tool for two reasons. Least squares residuals have the benefit of being easier to interpret and understand. More importantly, the strength of the BIC is that it penalizes models for having more parameters, even if more parameters produce a better fitting, because 
increasing the number of parameters also decreases the probability that the parameters used are statistically accurate. This may be important for larger models, but, for our purposes, all models either had one or two parameters, making this feature redundant. It could also be argued that two parameters is the least that a biologically viable tumor growth model can have, given that tumor growth is limited by the amount of available nutrients in the local area, so a carrying capacity must always exist. This is why the ranked model evaluation takes the least squares residuals into account more strongly than the BIC values for each trial.

Another relation between the evaluation metrics to take into account is whether the models with the lowest least squares residuals also had the lowest BIC values. As demonstrated in Table 3.2, the trial with the lowest least squares residuals also had the lowest BIC values in $65 \%$ of the cases, while the two metrics disagreed on $35 \%$ of the cases. While this nontrivial behavior is expected, given that the function that generates BIC values is a nonlinear function over the least squares residuals of a model, this difference serves to highlight the fact that choosing a different model evaluation metric would produce a different ranked ordering of models. 



\section{Chapter 5}

\section{Future Work}

\subsection{ODE models for CD47 Treatment}

To develop an ODE model accounting for CD47 treatment, we must first determine which components of the immune system are affected by CD47 suppressors. Willingham et al. reports that CD47 prevents phagocytosis of tumor cells by binding to $\operatorname{SIRP} \alpha$, a protein expressed on the surface of both macrophages and dendritic cells (Willingham, Stephen B. et al., 2012). Blocking the CD47 protein greatly increases the rate of phagocytosis of tumor cells by macrophages. In addition, anti-CD47 treatment increases the rate of activation by dendritic cells of tumor-specific cytotoxic lymphocytes (CTLs). Therefore, along with the usual cell types accounted for in tumorimmune system interaction models; natural killer (NK) cells, CTLs; we must also account for changes in the dendritic cell and macrophage populations. In addition, anti-CD47 treatment is a monoclonal antibody (MAB) treatment, requiring an additional term for treatment concentration.

We begin with a simplified model from de Pillis 2005, which accounts for tumor growth, NK cell populations, and CTL populations (de Pillis et al., 2005). Although future models published by the same authors have also included interleuken-2 (IL-2) dynamics, accounting for a chemical signal that affects CTL proliferation, there are many immunotherapies that affect the IL-2 concentration (de Pillis et al., 2013a, 2009, 2013b). In this model, because the particular immunotherapy we are studying affects IL-2 concentration only indirectly through the proliferation of other cell lines, we reference it only indirectly and remove the IL-2 equation. We let $T(t)$ represent the tumor population, $N(t)$ represent the $N K$ cell population, and $L(t)$ represent the CTL population. The original model, from de Pillis 2005, 
is given as

$$
\begin{aligned}
\frac{d T}{d t} & =\gamma(T)-c N T-D \\
\frac{d N}{d t} & =\sigma-f N+\frac{g T^{2}}{h+T^{2}} N-p N T \\
\frac{d L}{d t} & =-m L+\frac{j D^{2}}{k+D^{2}} L-q L T+r N T
\end{aligned}
$$

where

$$
D=d \frac{(L / T)^{\lambda}}{s+(L / T)^{\lambda}} T
$$

and $\gamma(T)$ is the ODE tumor growth equation which was determined to be the best-fit model for the target type of cancer by our previous parameter fittings. To account for dendritic cell dynamics, we can include a new dendritic cell paper with all of the compartments (de Pillis et al., 2013b). Our macrophage dynamics depend both on the total macrophage population, the localized macrophage population near the tumor cell, and the concentration of chemoattractant near the tumor cell. The macrophage population and chemoattracant concentration, denoted $M$ and $W$, respectively, are based on a model from Byrne et al. 2004 (Byrne, H.M. and Cox, S.M. and Kelly, C.E., 2004):

$$
\begin{aligned}
\frac{d M}{d t} & =(M *-M) \frac{a W}{1+b W}-u M(T+M+v) \\
\frac{d W}{d t} & =w T-x W .
\end{aligned}
$$

This is where we encounter a significant challenge, however, because Byrne et al. did not have sufficient data to determine biologically accurate parameters, making most of the macrophage-associated constants unusable for modeling purposes. While the decision to estimate parameters without experimental data did allow Byrne et al. to evaluate the macrophage model as a dynamical system, the current state of the model cannot be used to verify tumor-immune system behavior under the influence of various medicines. In addition, a thorough literature search revealed that experimental data relating directly to the macrophage parameters was either difficult to find or nonexistent. Therefore, producing a biologically motivated CD47 model would require large parts of the model to be built from scratch, which was not possible in the time period given for this project. 
Despite the difficulty inherent in attempting this project, modeling tumorimmune system interaction with CD47 treatment is extremely attractive because the treatment acts in a way that is relatively novel to the field of cancer therapy, as previously stated. One of the implications of building a CD47 model is the inclusion of tumor types that are not normally associated with successful reactions to immunotherapies. Out of all of the cancer types, only renal cell carcinoma and melanoma have been shown to have success with CD8+ and interleuken-affecting immunotherapies; however, as CD47 treatment has been shown to affect ovarian, breast, colon, bladder, and prostate cancer, as well as glioblastoma and hepatocellular carcinoma, it is necessary for ODE model developers to produce tumor growth parameters for an ever-increasing number of tumor types (de Pillis et al., 2013a, 2009, 2013b; Willingham, Stephen B. et al., 2012). The results of this project make these efforts almost trivial, so that future dynamical systems researchers need only develop the parameters for macrophage and dendritic cell terms. 



\section{Appendix A}

\section{Supplemental Materials}

\section{A.1 Sources of Data for Parameter Values}

A large number of individual studies were gathered in determining appropriate timescale tumor growth data sets to be used in the fitting process. Not only are the sources for each type of cancer listed, the individual cell lines used in each paper are included for posterity. Some papers, which used tissue samples from human subjects as the source of cancerous cells, did not specify a cell line.

\begin{tabular}{ll}
\hline Cancer and Cell Line & Sources \\
\hline Bladder Cancer & \\
HT1376 & Golshani et al. (2008) \\
UMUC-3 & Kamada et al. (2007) \\
KoTCC-1 & Miyake et al. (2001) \\
EJ-1 & Ohnishi et al. (2003); Du and Hou (2003) \\
Breast Cancer & \\
MDA-MB-435BAG & Coopman et al. (2000) \\
MCF-7 & Lu and Serrero (1999) \\
KPL-1 & Nakagawa et al. (2001) \\
4T1-GFP-FL & Smith et al. (2004) \\
Colon Cancer & \\
KM12L4 & Reinmuth et al. (2002) \\
Moser & Sarraf et al. (1998) \\
HCT116 & Sarraf et al. (1998); Sheng et al. (1997) \\
CX-1 & Sarraf et al. (1998) \\
\hline
\end{tabular}

Table A.1 Sources of Timescale Data by Type of Cancer and Cell Line 
38 Supplemental Materials

\begin{tabular}{|c|c|}
\hline Cancer and Cell Line & Sources \\
\hline HCA7 & Sheng et al. (1997) \\
\hline LS LiM6 & Warren et al. (1995) \\
\hline Unspecified & Todaro et al. (2007) \\
\hline \multicolumn{2}{|c|}{ Head and Neck Squamous Cell Carcinoma } \\
\hline UM-SCC-9 & Duffey et al. (1999) \\
\hline Tu-138 & Liu et al. (1999) \\
\hline Tu-167 & Liu et al. (1999) \\
\hline $686 \mathrm{LN}$ & Liu et al. (1999) \\
\hline CAL27 & LoTempio et al. (2005) \\
\hline UM-SCC-X & Ricker et al. (2004) \\
\hline PAM-LY2 & Sunwoo et al. (2001) \\
\hline \multicolumn{2}{|c|}{ Hepatocellular Carcinoma } \\
\hline HCC-26-1004 & Huynh et al. (2008) \\
\hline HCC-2-1318 & Huynh et al. (2008) \\
\hline SH-J1 & Jung et al. (2006) \\
\hline PLC & Liu et al. (2005) \\
\hline Hep3B & Liu et al. (2005) \\
\hline SMMC-7721 & Wong et al. (2005) \\
\hline Unspecified & Zender et al. (2008) \\
\hline \multicolumn{2}{|l|}{ Lung Cancer } \\
\hline SW-900 & Esquela-Kerscher et al. (2008) \\
\hline $\mathrm{H} 226$ & Esquela-Kerscher et al. (2008) \\
\hline \multirow[t]{3}{*}{ A549 } & Esquela-Kerscher et al. (2008) \\
\hline & Fabbri et al. (2005) \\
\hline & Tsubouchi et al. (2000) \\
\hline $\mathrm{H} 460$ & Fabbri et al. (2005) \\
\hline H1299 & Fabbri et al. (2005) \\
\hline U2020 & Fabbri et al. (2005) \\
\hline $\mathrm{H} 322 \mathrm{a}$ & Fujiwara et al. (1993) \\
\hline WT226b & Fujiwara et al. (1993) \\
\hline NCI-H727 & Moody et al. (1993) \\
\hline 3LL & Sharma et al. (1999) \\
\hline NCI-H358 & Takahashi et al. (1992) \\
\hline H841 & Tsubouchi et al. (2000) \\
\hline pc14 & Tsubouchi et al. (2000) \\
\hline \multicolumn{2}{|l|}{ Melanoma } \\
\hline M3Dau & Boukerche et al. (1989) \\
\hline
\end{tabular}

Table A.1 Sources of Timescale Data by Type of Cancer and Cell Line 


\begin{tabular}{|c|c|}
\hline Cancer and Cell Line & Sources \\
\hline MIRW5 & Bregman et al. (1986) \\
\hline B16-BL6 & Caltagirone et al. (2000); Murgo (1985) \\
\hline A-375 & Kunstfeld et al. (2003) \\
\hline M21 & Petitclerc et al. (1999) \\
\hline $\mathrm{Hs} 0294$ & Richmond et al. (1983) \\
\hline Unspecified & Abe et al. (2004) \\
\hline \multicolumn{2}{|l|}{ Ovarian Cancer } \\
\hline SKOV-3 & Juhl et al. (1997); Polato et al. (2005) \\
\hline HRA & Nakata et al. (1998) \\
\hline A2780 & Polato et al. (2005) \\
\hline IGROV-1 & Polato et al. (2005) \\
\hline НCТ-116 & Polato et al. (2005) \\
\hline MA148 & Yokoyama et al. (2000) \\
\hline \multicolumn{2}{|l|}{ Pancreatic Cancer } \\
\hline PC-1 & Burke et al. (1997) \\
\hline MIAPaCa-2 & Ito et al. (1996); Kisfalvi et al. (2009) \\
\hline PANC-1 & Kisfalvi et al. (2009) \\
\hline PancTu1 & Vogler et al. (2009) \\
\hline HPAC & Zervos et al. (1997) \\
\hline \multicolumn{2}{|l|}{ Renal Cell Carcinoma } \\
\hline $786-\mathrm{O}$ & $\begin{array}{l}\text { Dhanabal et al. (1999); Lieubeau-Teillet } \\
\text { et al. (1998) }\end{array}$ \\
\hline $\mathrm{ACHN}$ & Huang et al. (2008) \\
\hline A-498 & Huang et al. (2008) \\
\hline \multirow[t]{2}{*}{ Caki-1 } & Inoue et al. (2001); Schirner et al. (1998) \\
\hline & Shi and Siemann (2002) \\
\hline SK-RC-29 & Prewett et al. (1998) \\
\hline Caki-2 & Schirner et al. (1998) \\
\hline Unspecified & Fujimoto et al. (1995) \\
\hline
\end{tabular}

Table A.1 Sources of Timescale Data by Type of Cancer and Cell Line 


\section{A.2 Results of Parameter Fittings}

Individual data sets are labeled with the year and author, and given a unique identifier: either the label they were presented with in the figure from which the data originated, or the cell line that is used in the paper.

In some cases, the line representing the result of the parameter fitting is not visible. This happens for one of two reasons: a large difference between orders of magnitude in separate data sets, limiting the available space for data sets with smaller orders of magnitide; or because two or more data sets started with the same initial condition, causing the combined fitting result to produce the same curve. A complete list of all of the parameter fittings that are not visible, and the reason for why they cannot be seen, is given below:

- In the combined in vitro bladder cancer trials, the AS clustering and MM control trials of Miyake 2001 share an initial condition, hence only the MM control fitting is visible.

- In the combined in vitro breast cancer trials, the three Smith 2004 trials share the same initial condition, so the purple curve indicates the fitting to all three of these trials.

- In the combined in vivo breast cancer trials, the two Coopman 2000 trials share an initial condition, thus the green curve represents the fitting to both trials.

- In the combined in vitro colon cancer trials, the Moser and HCT116 trials have the same initial condition, so the green curve represents the combined fitting to both.

- In the combined in vivo colon cancer trials, two sets of trials have the same initial condition-the two Reinmuth 2002 trials and the two Warren 1995 trials. As a result, the orange curves account for both Reinmuth 2002 trials and the pink curves to both Warren 1995 trials.

- In the combined in vivo head and neck squamous cell carcinoma trials, the three Liu 1999 trials start with the same initial conditions, hence the teal curve represents the combined fitting to all three data sets.

- In the combined in vitro hepatocellular carcinoma trials, the Huynh 2008 trials share an initial condition, so the green curve represents the fitting to both data sets. 
- In the combined in vivo hepatocellular carcinoma trials, the Liu 2005 data sets have the same initial condition, so the teal curve indicates the fitting to both data sets.

- In the individual in vitro lung cancer trials, Fig. 4D from Fabbri 2005 was cropped from the graph because it was two orders of magnitude higher than the next largest tumor, making the other 12 trials impossible to distinguish. Despite its exclusion here, it was used in the fitting analysis.

- In the combined in vitro lung cancer trials, not only is Fig. $4 \mathrm{D}$ from Fabbri 2005 excluded, but several trials from the same study have the same initial conditions (i.e., the four visible Fabbri 2005 trials, SW900 and A549 from Esquela-Kerscher, and all three Fujiwara trials.) For this figure, the seafoam green curve is the fit for all 4 visible Fabbri 2005 trials, the SW-900 and A549 trials from Esquela-Kerscher are both represented by the yellow curve, and all three Fujiwara trials are represented by the purple curve. Additionally, for the Von Bertalanffy fitting, the data sets from Fujiwara 1993 and Takahashi 1992 are hidden by Fig. 3 from Tsubouchi 2000, presumably because their initial conditions are sufficiently close to each other.

- In the in vivo melanoma trials, the Boucherke 1989 trial is difficult to see because of its relatively low order of magnitude, but is visible along the bottom of the graphs.

- In the combined in vitro ovarian cancer trials, the A2780 and SKOV-3 trials have the same initial condition, and the IGROV-1 and HCT-116 trials have the same initial condition. As a result, the green curve represents the fitting to the first two trials, and the purple curve is the fitting to the last two trials. 

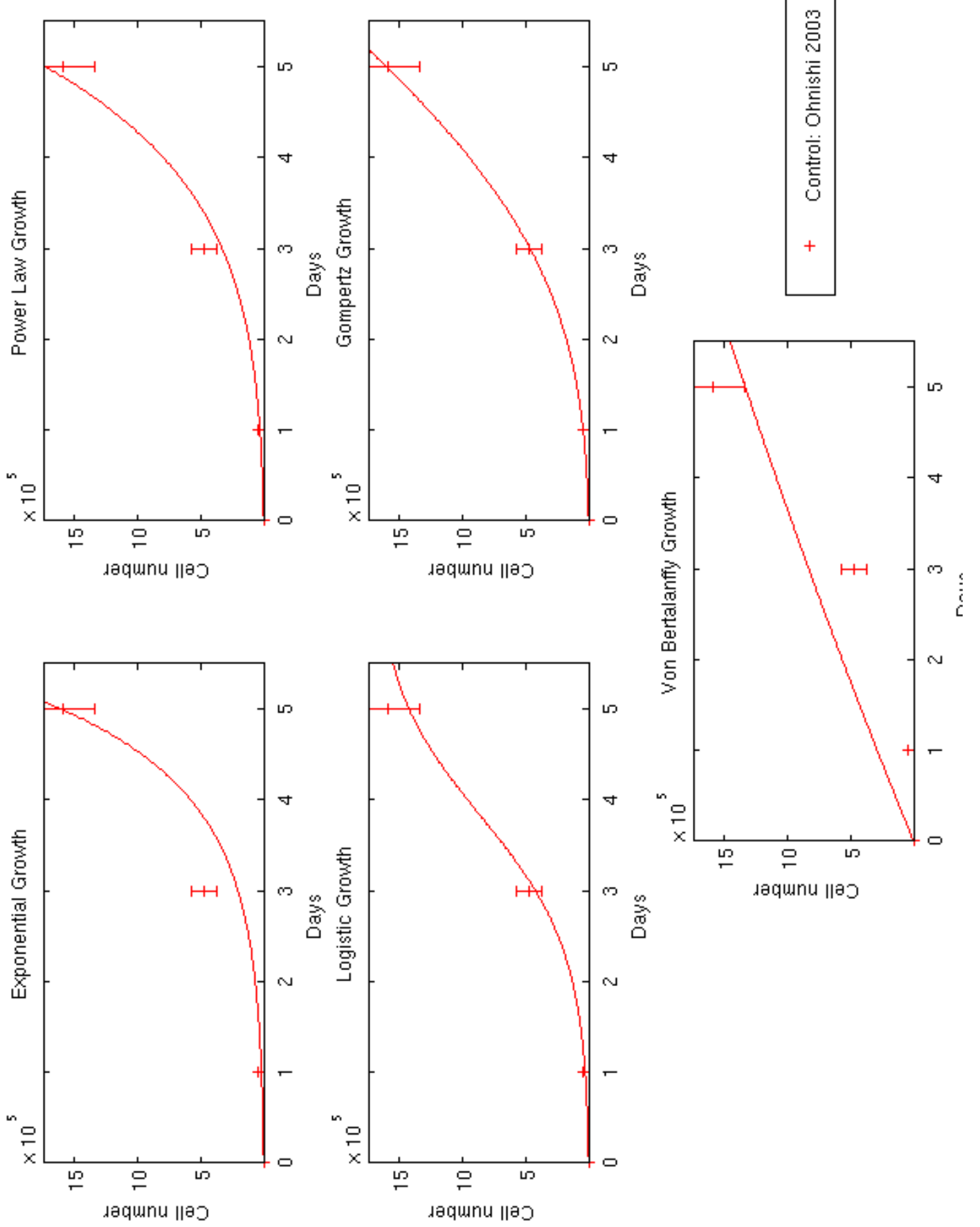


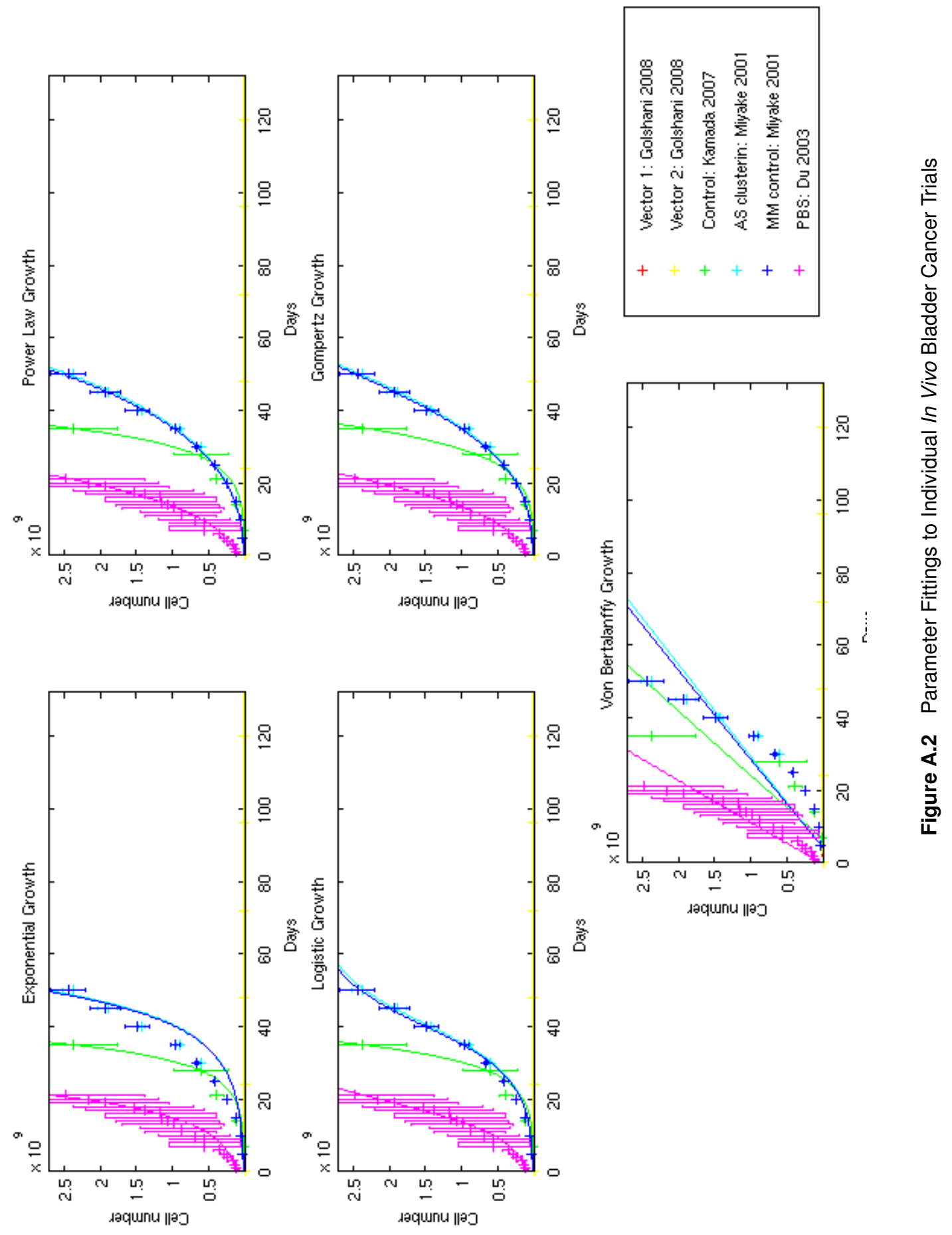




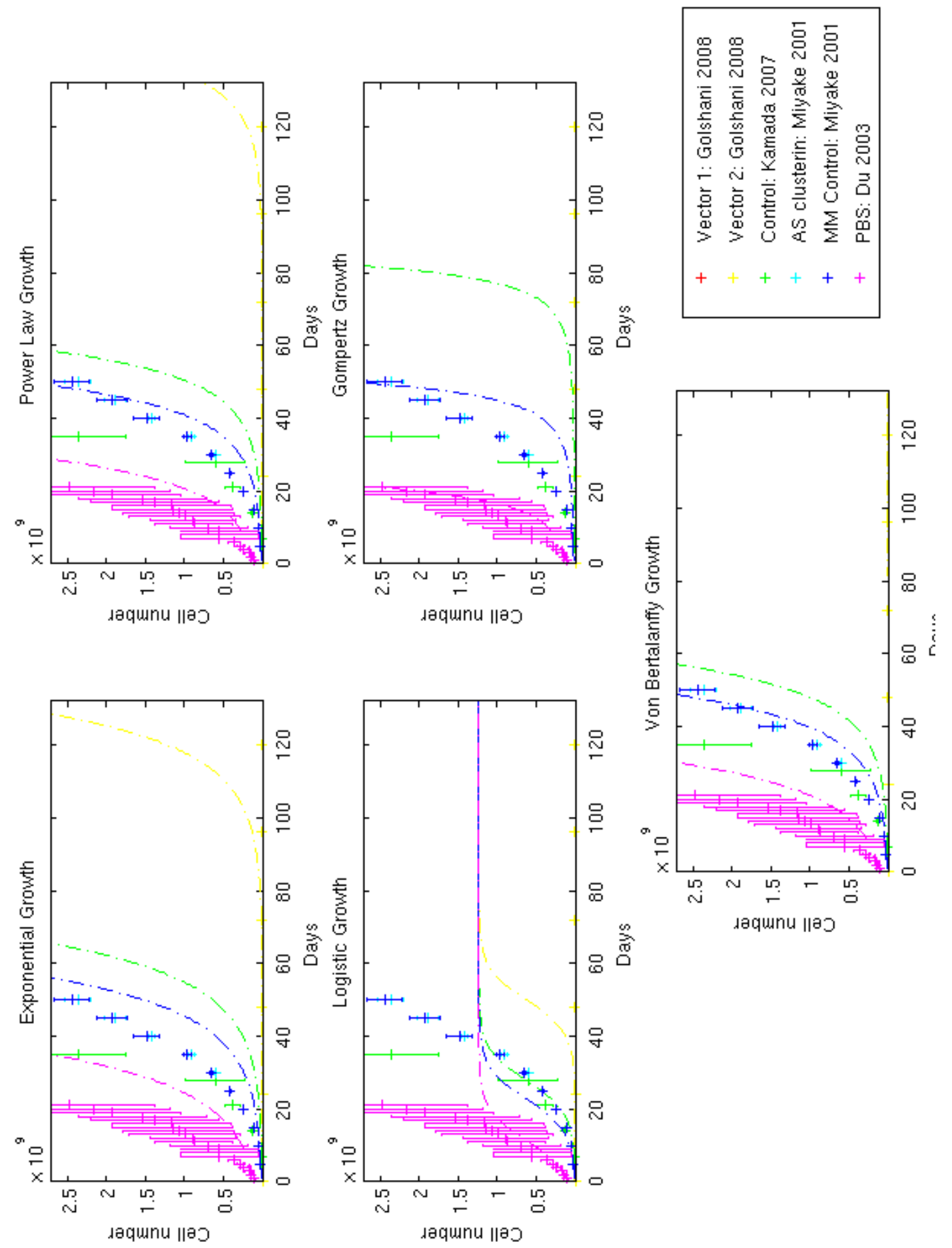



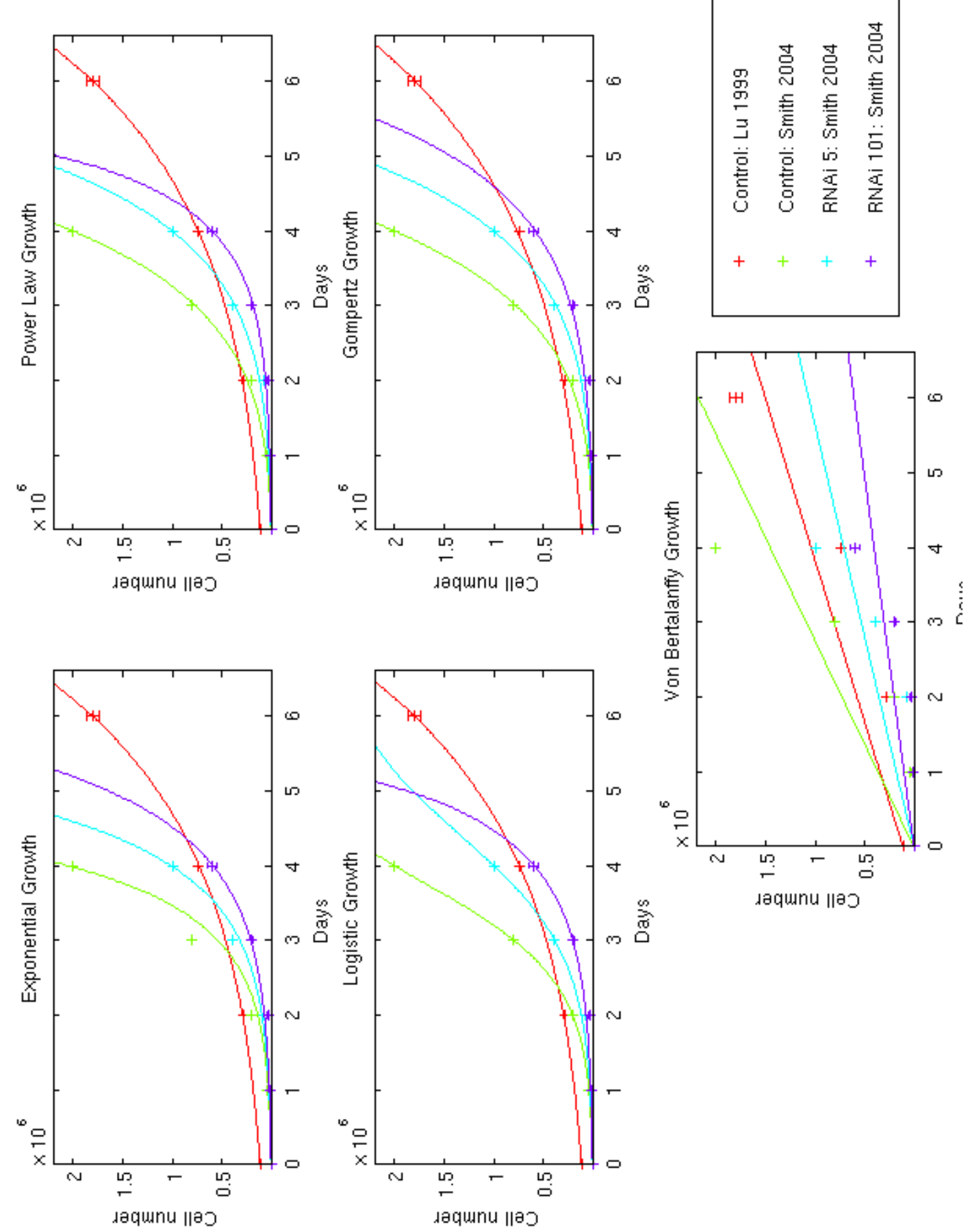

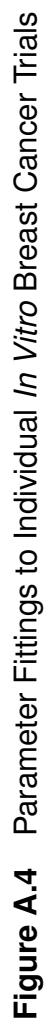



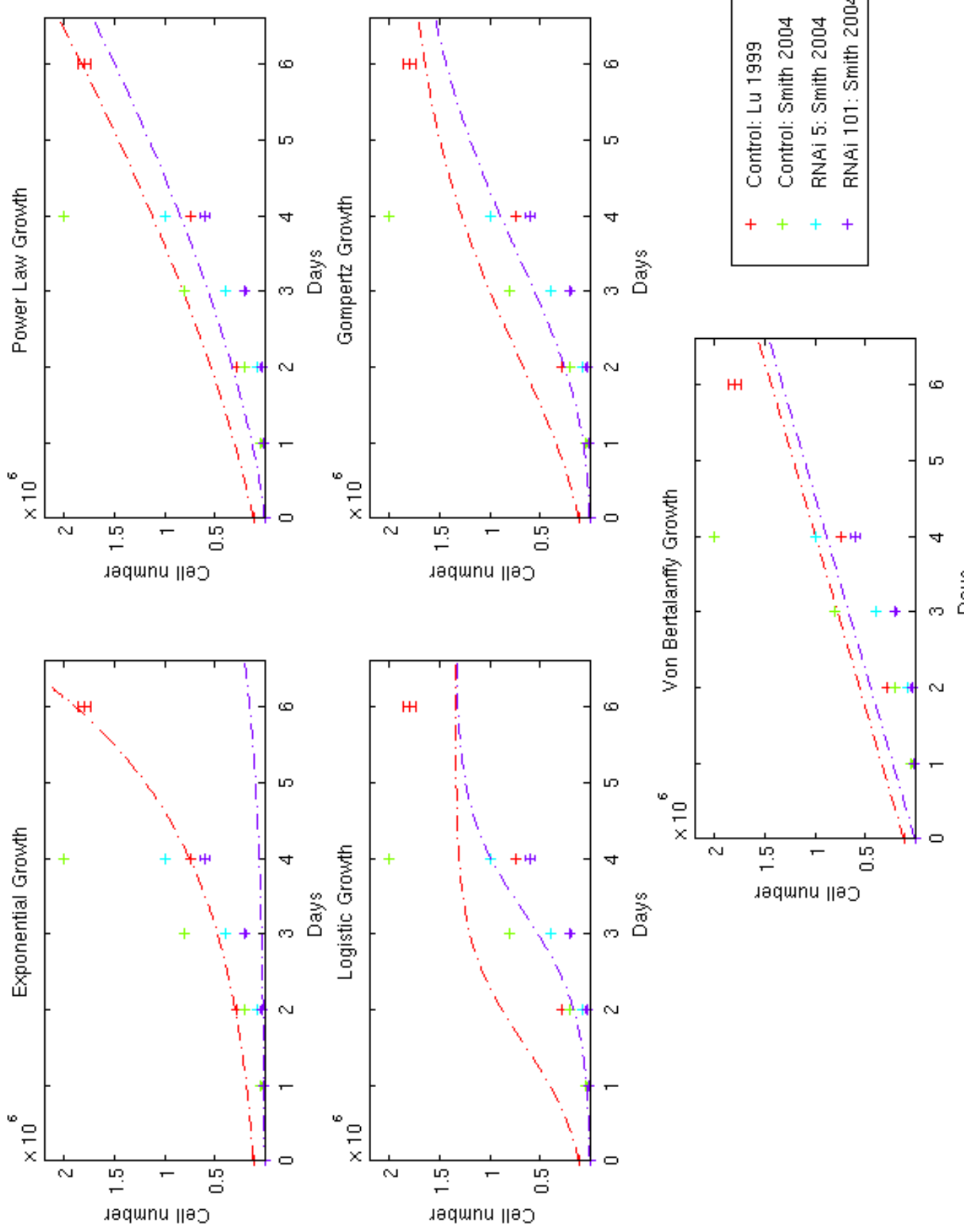

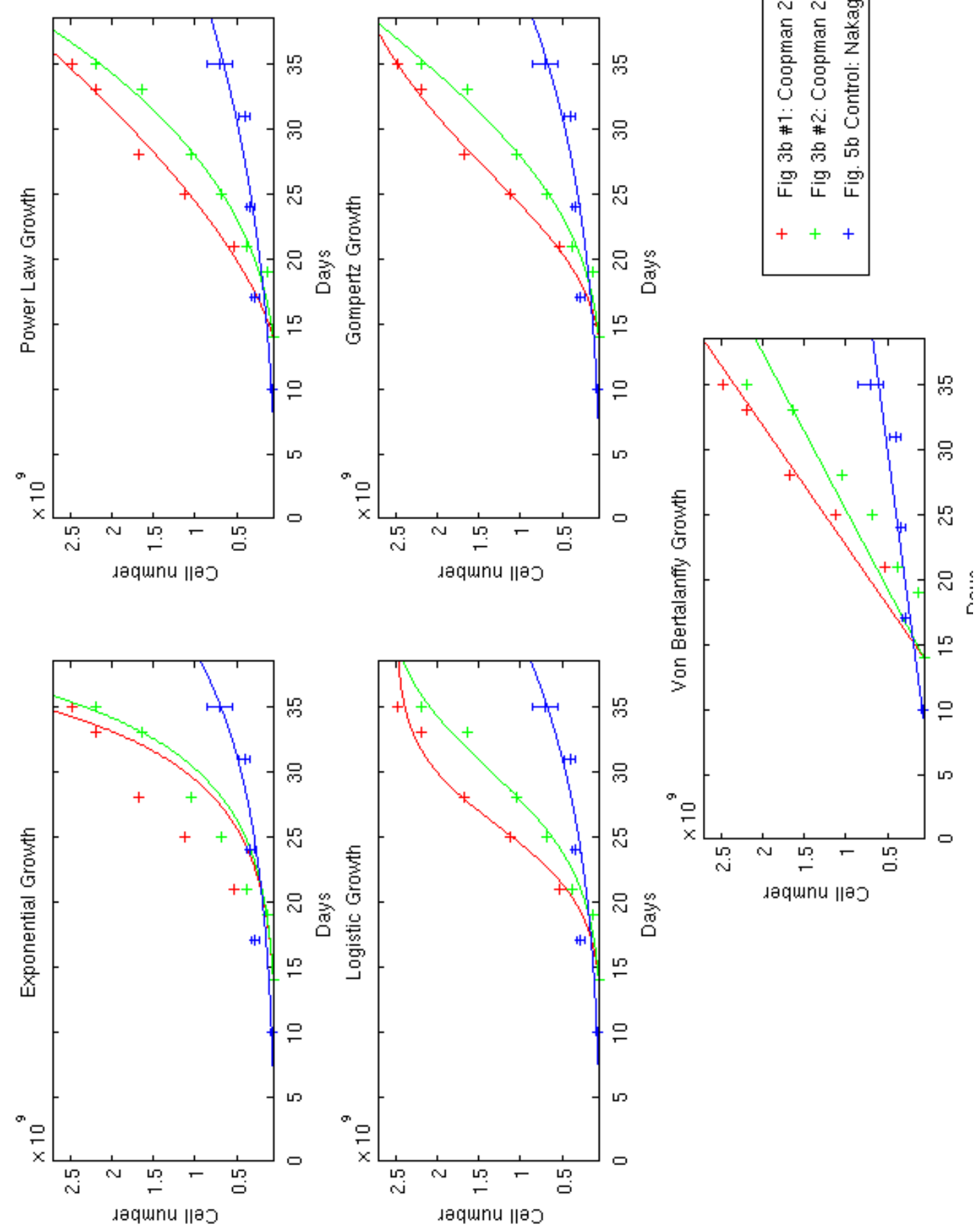

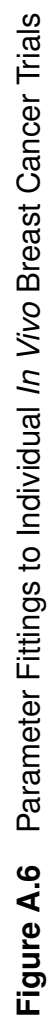




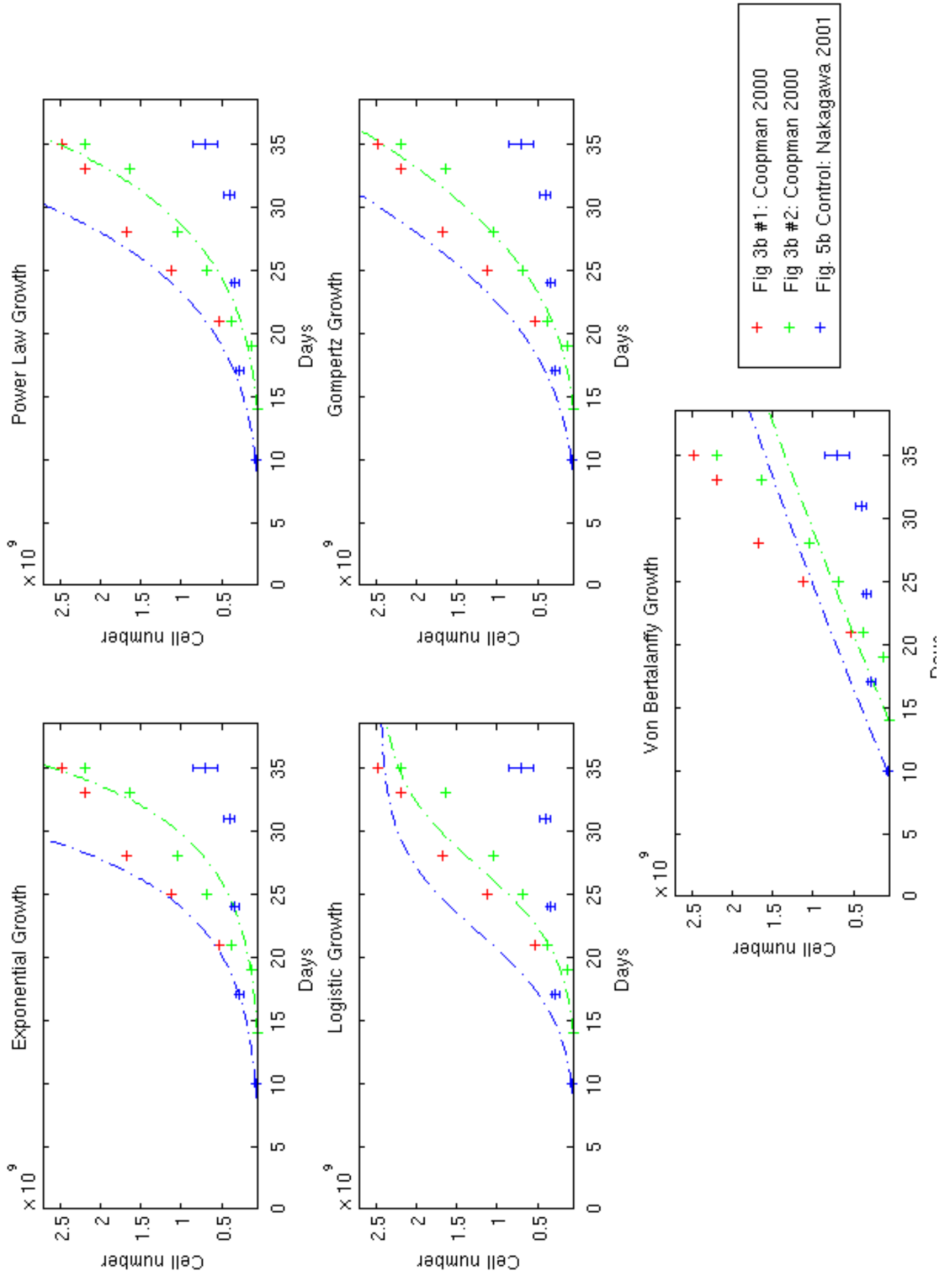



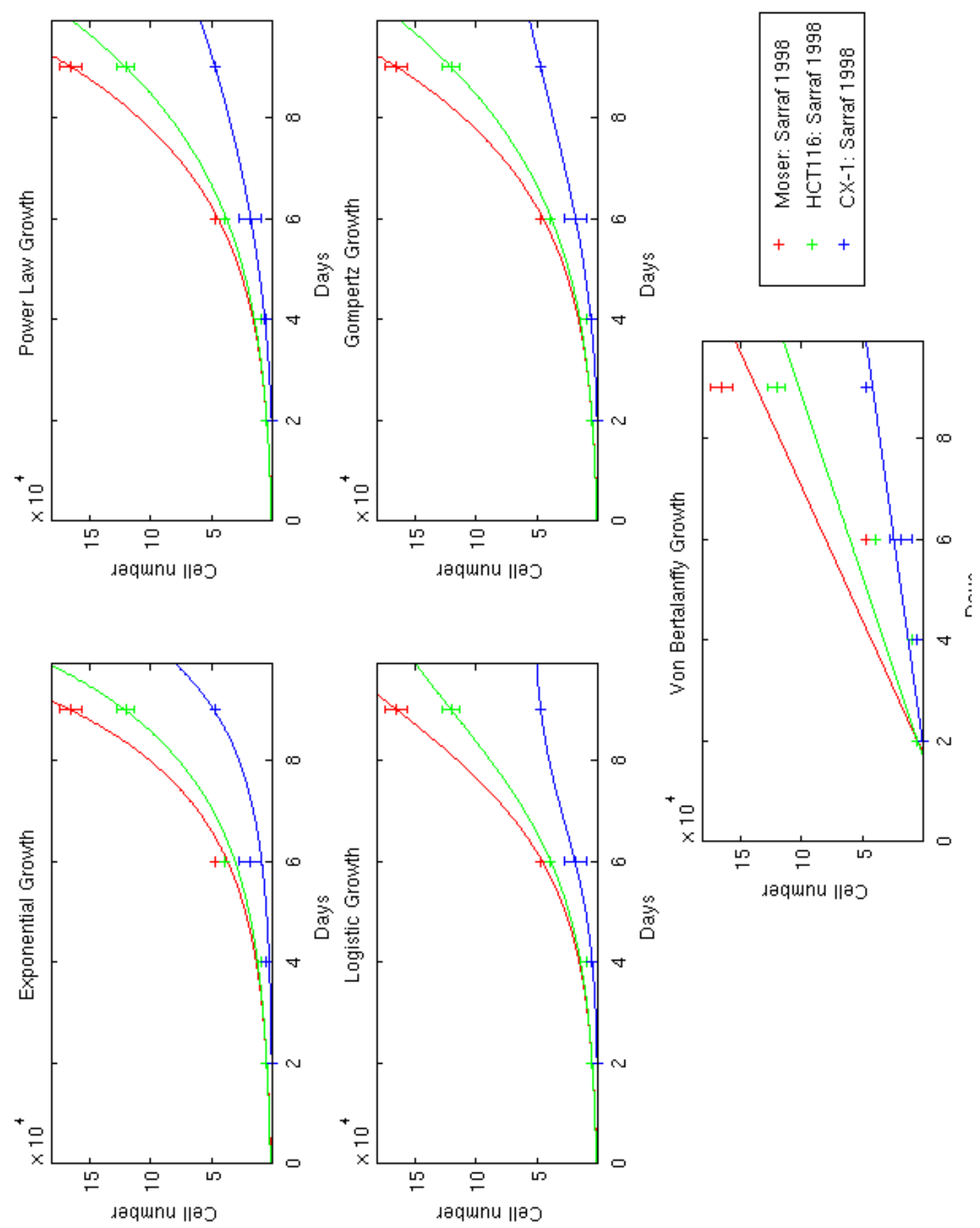

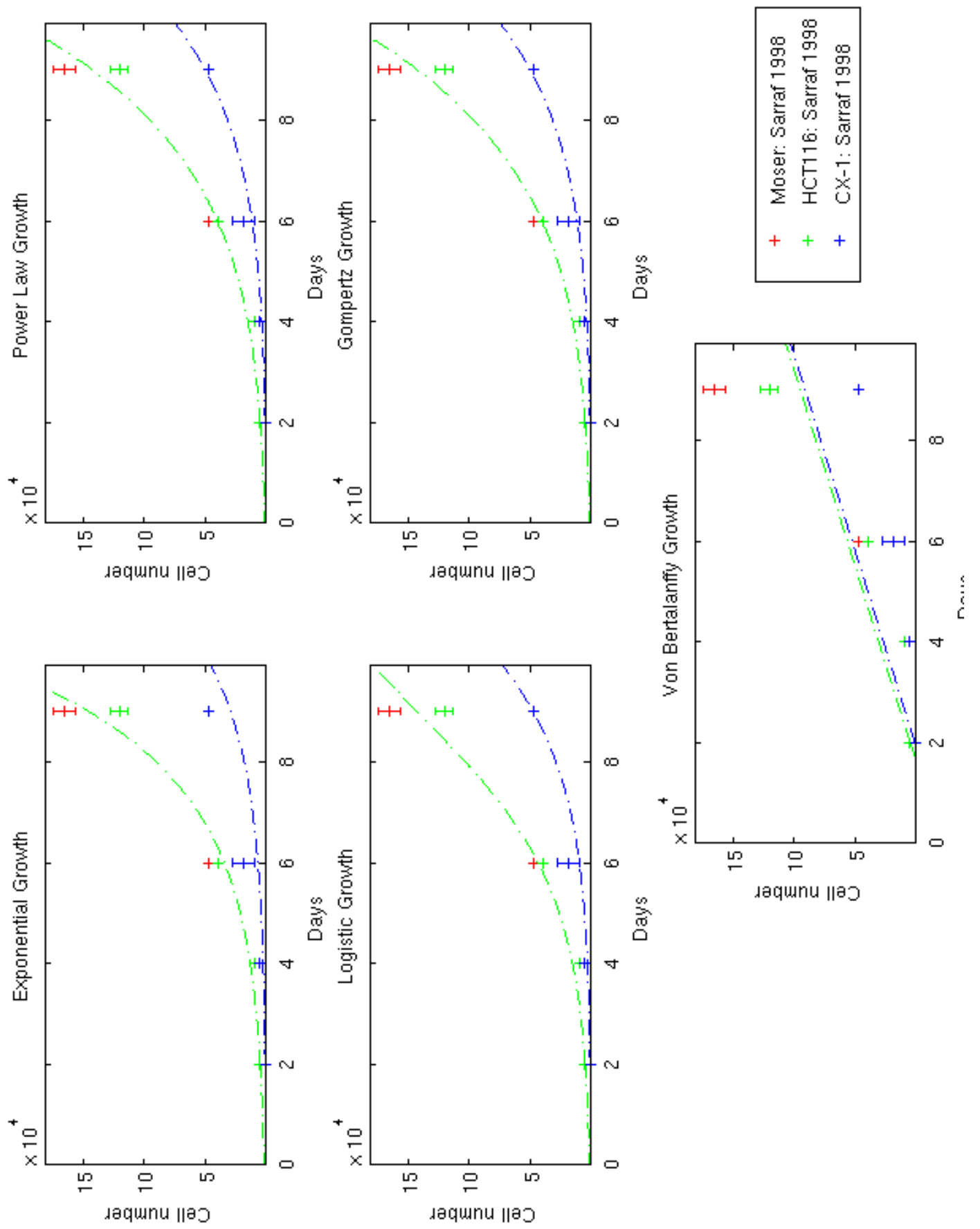


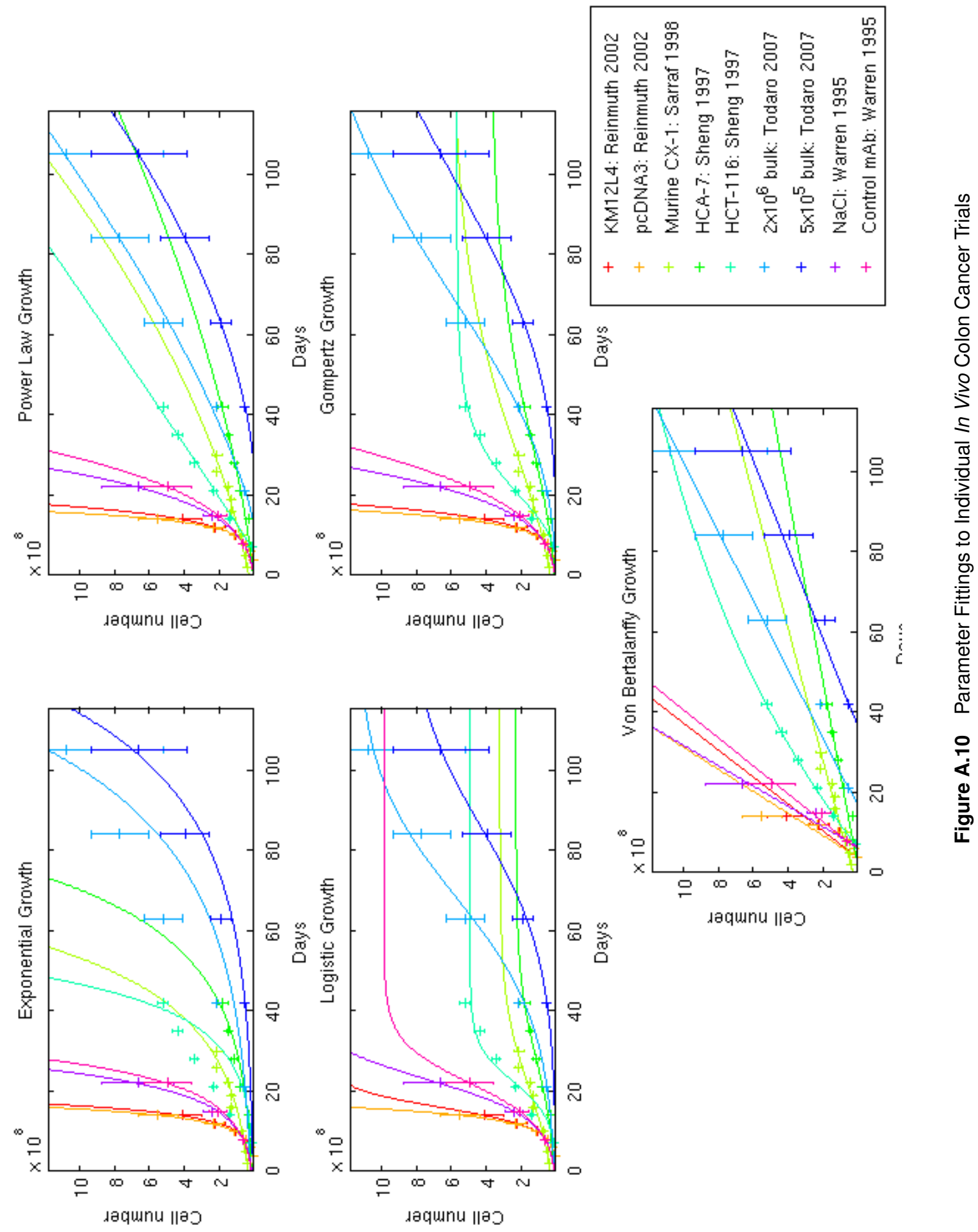




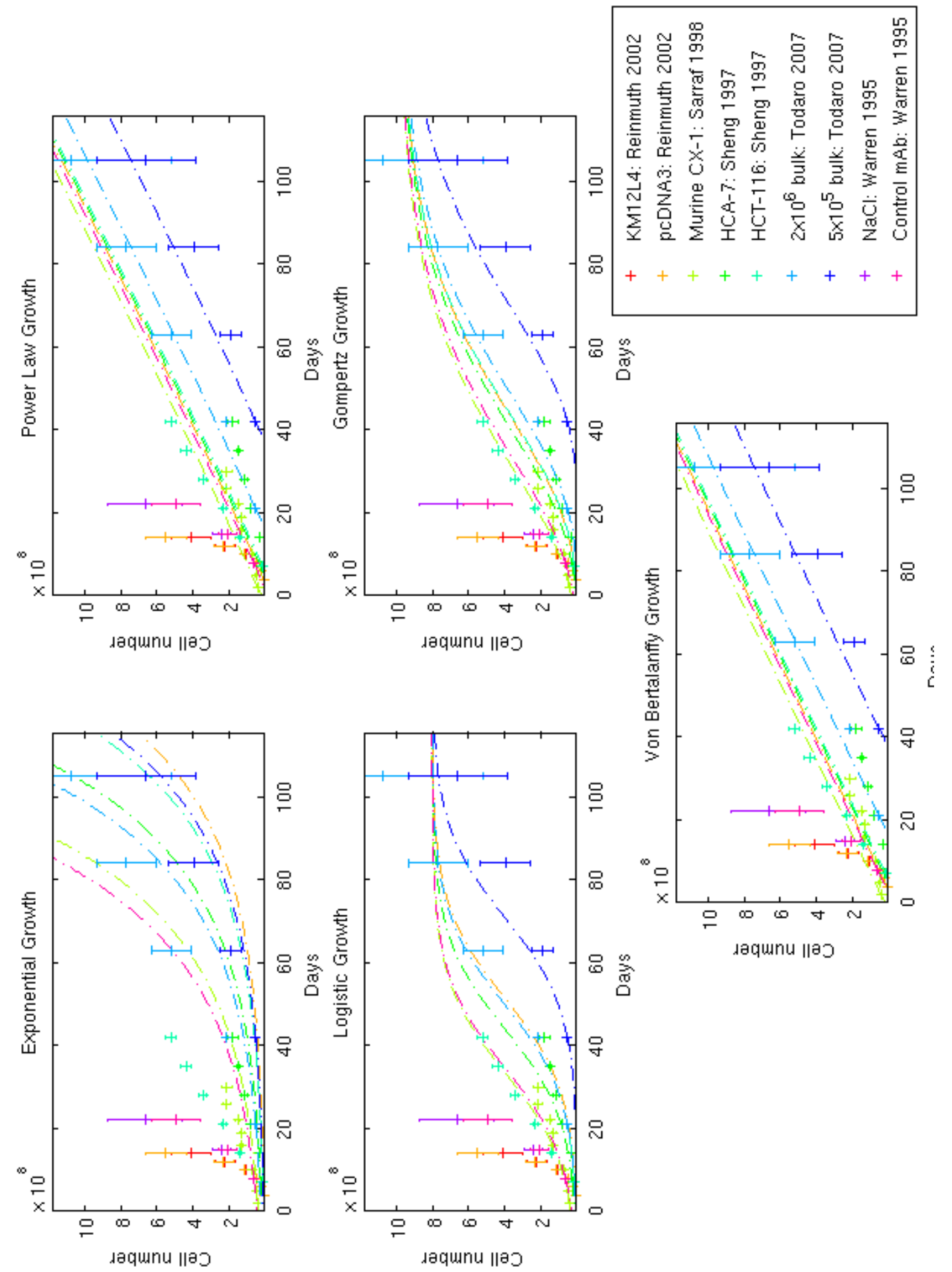

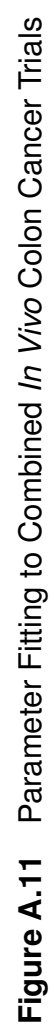



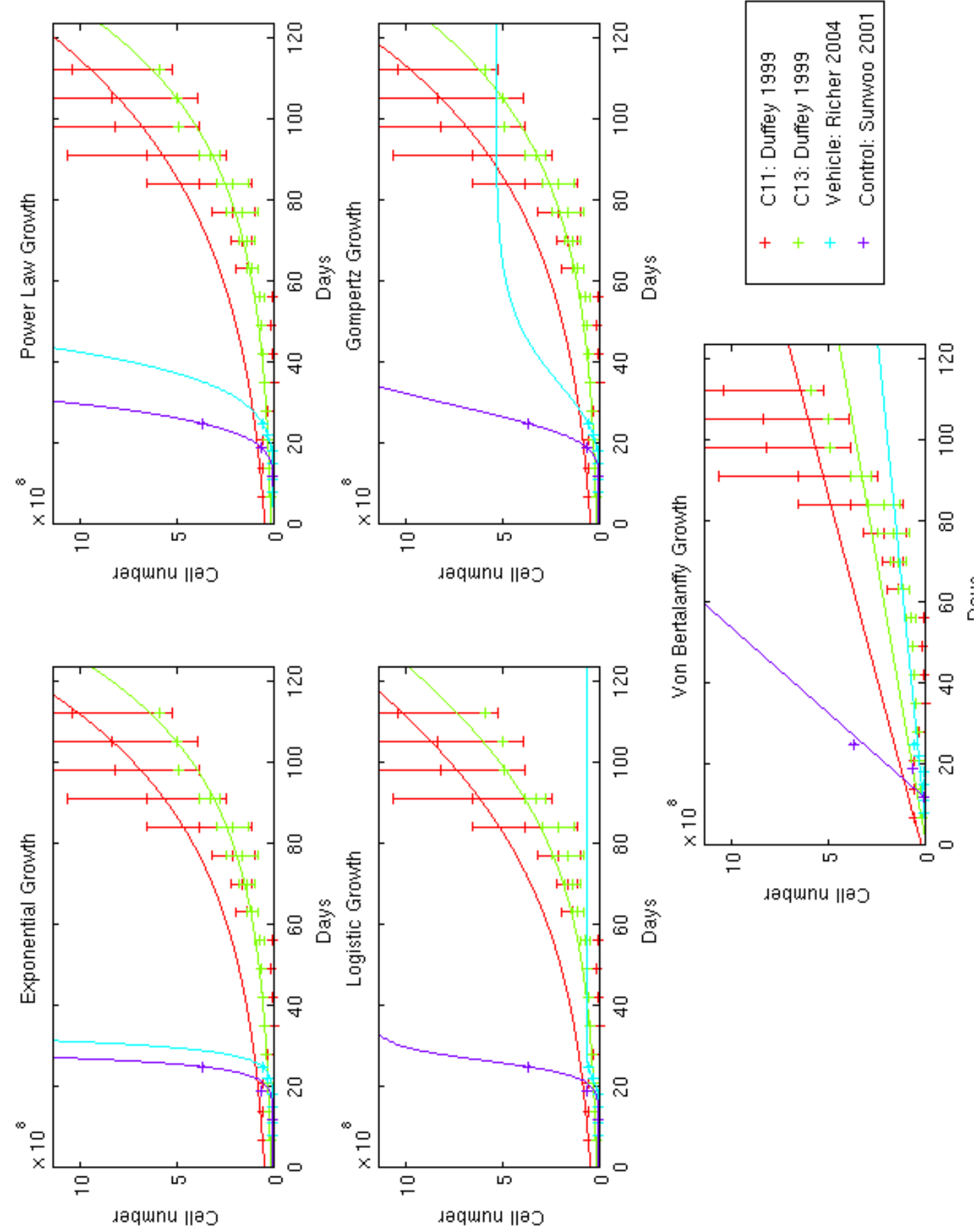

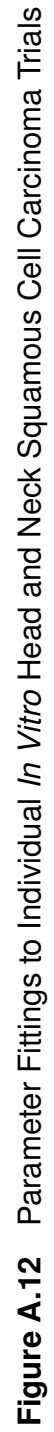



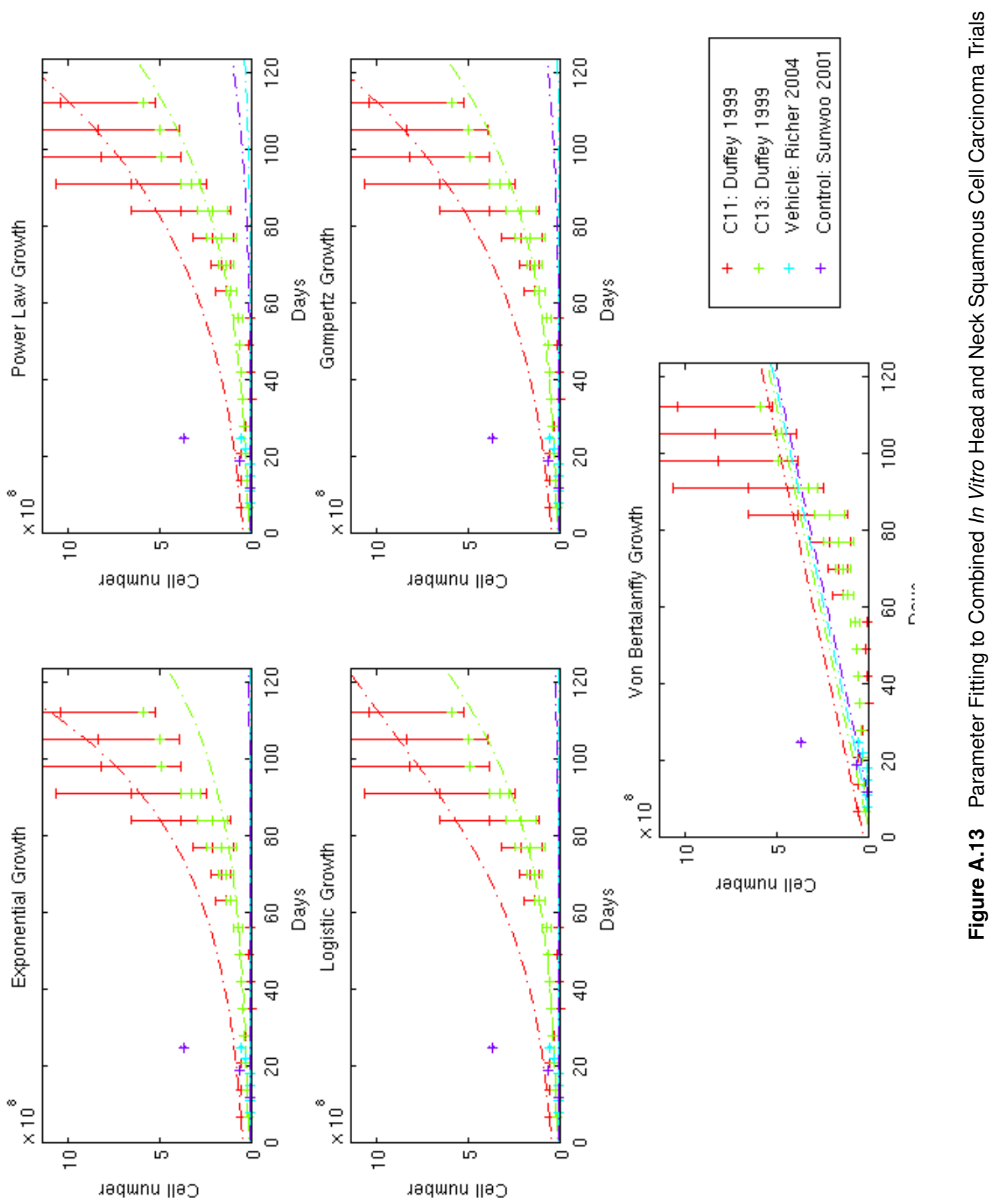

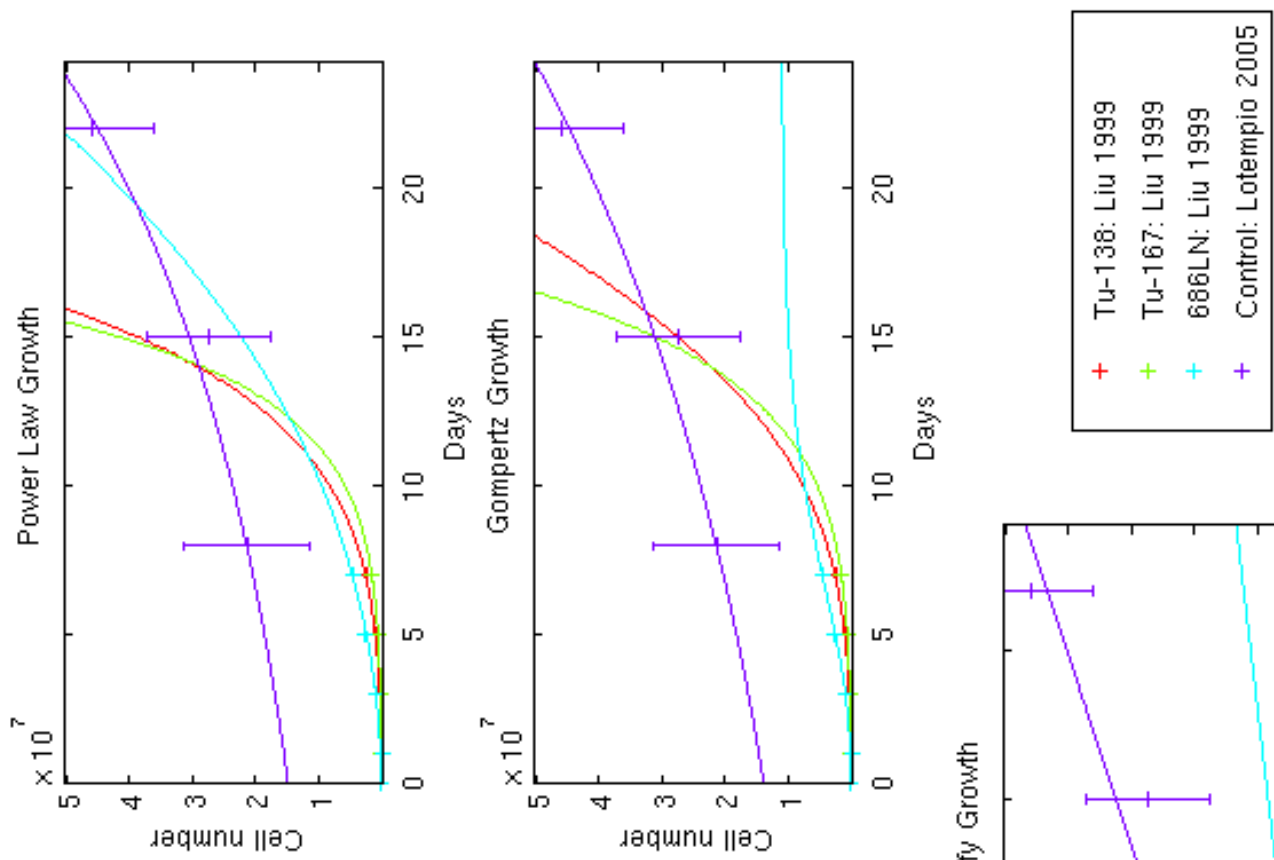

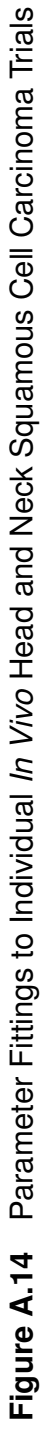
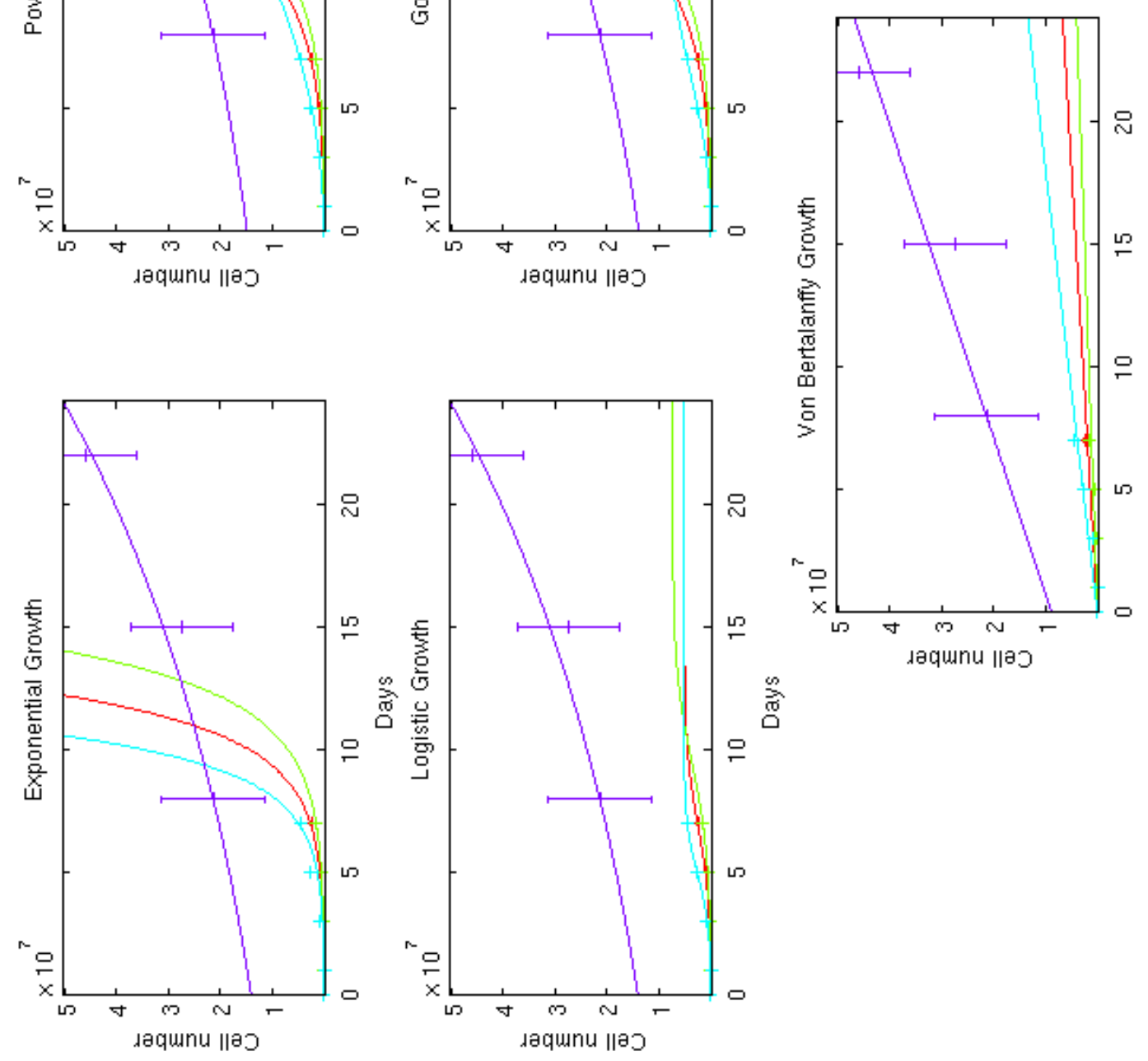

$\stackrel{\infty}{\circ}$ 

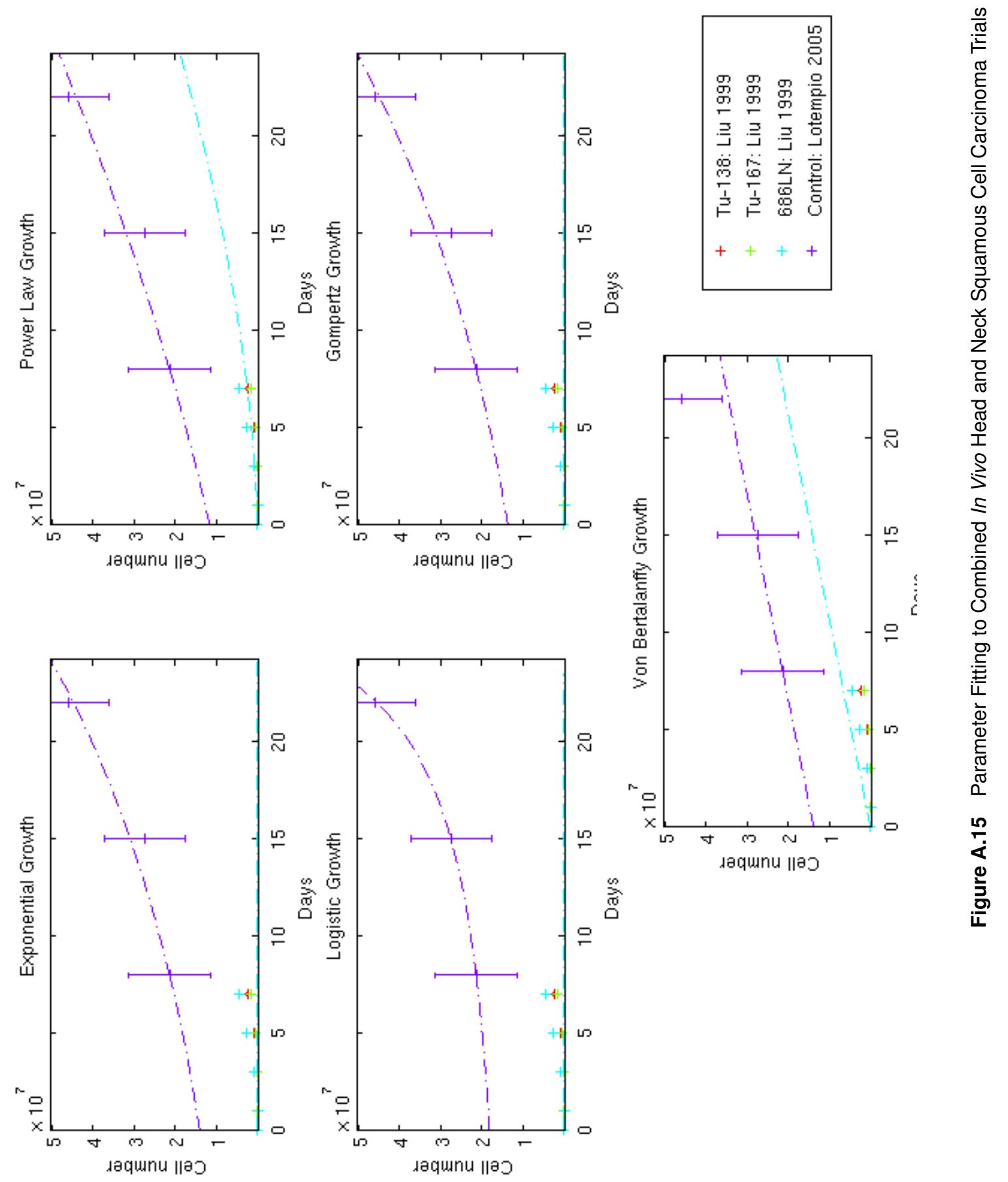


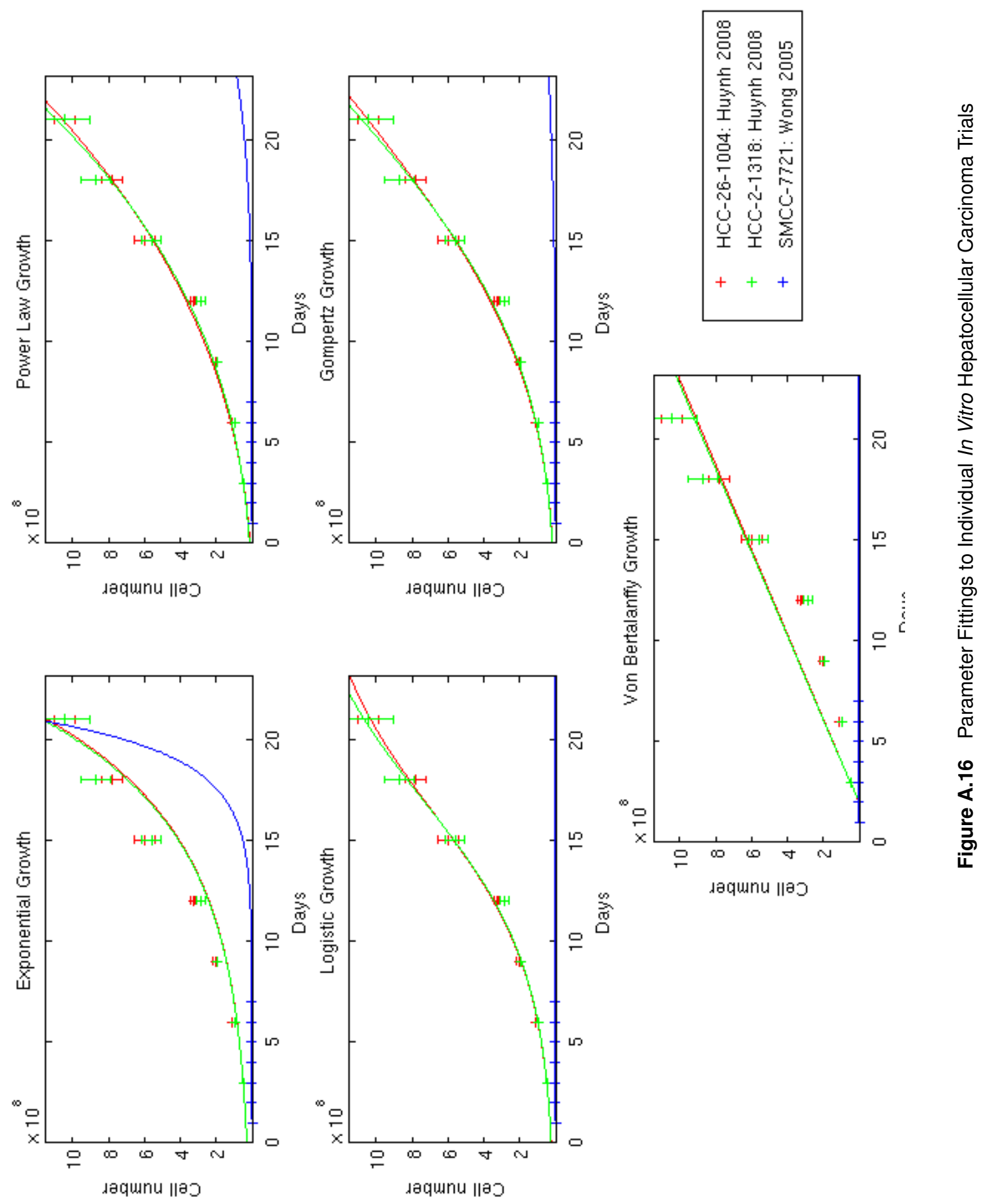




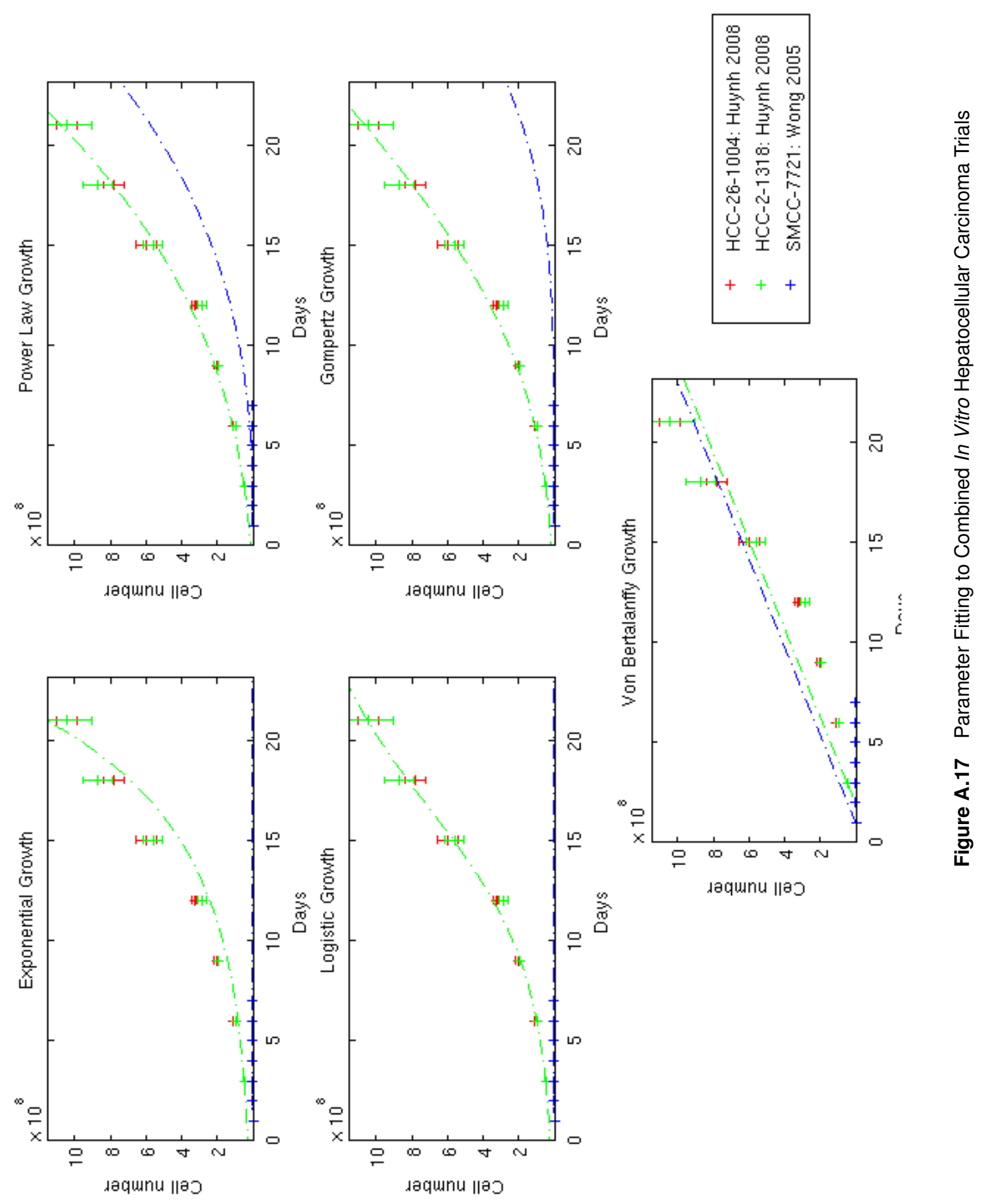



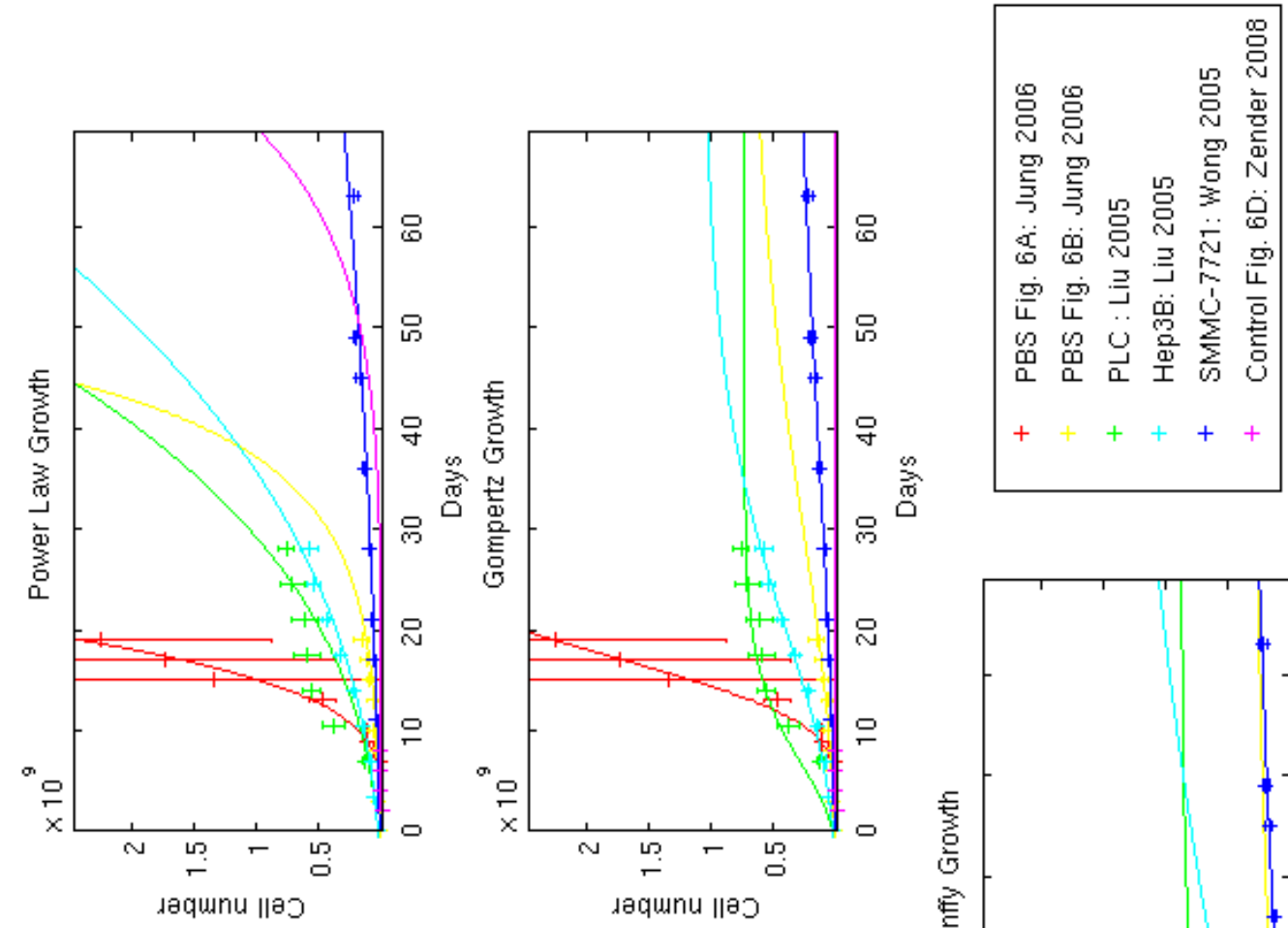

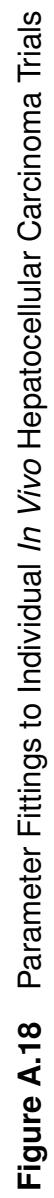
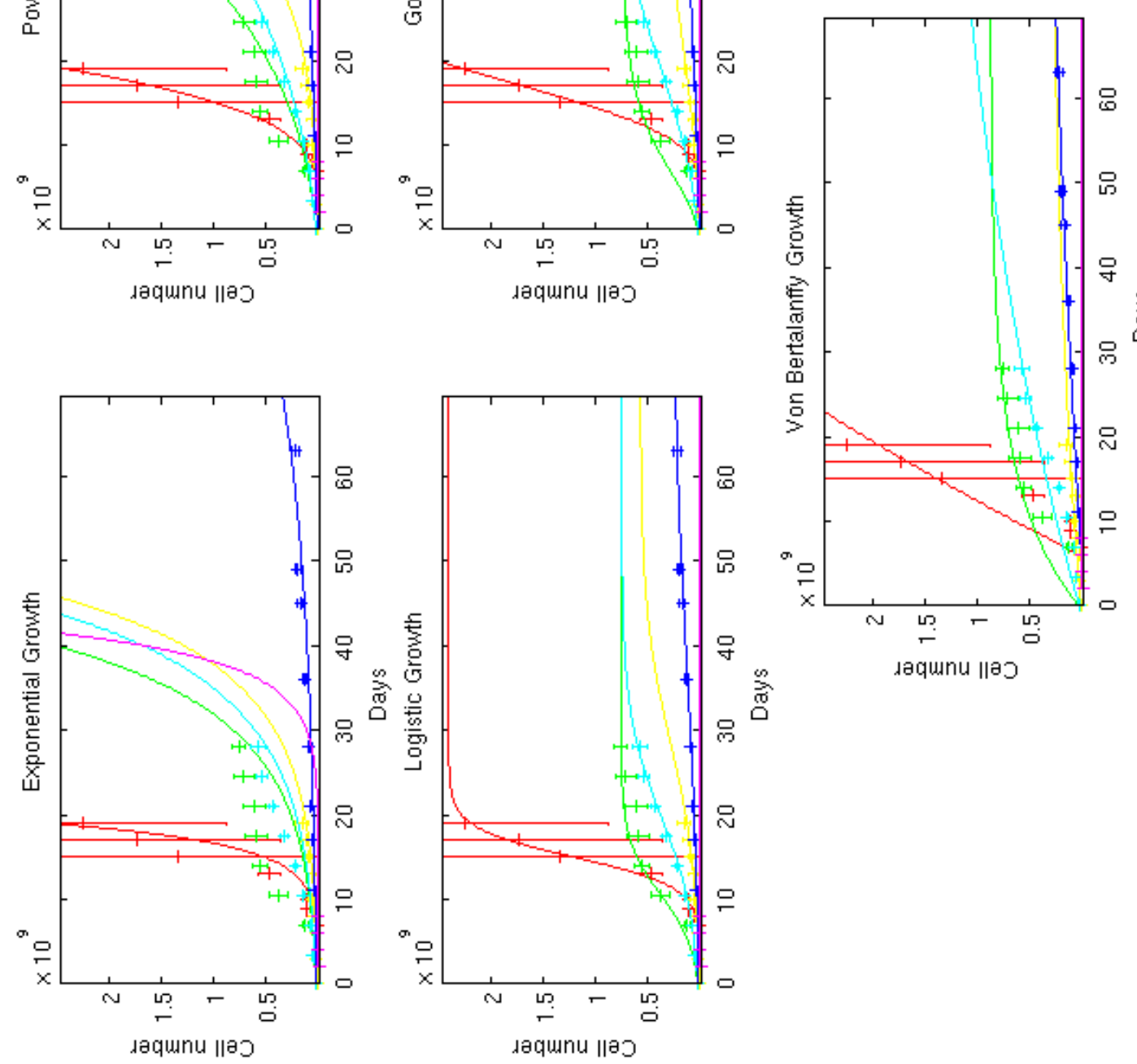


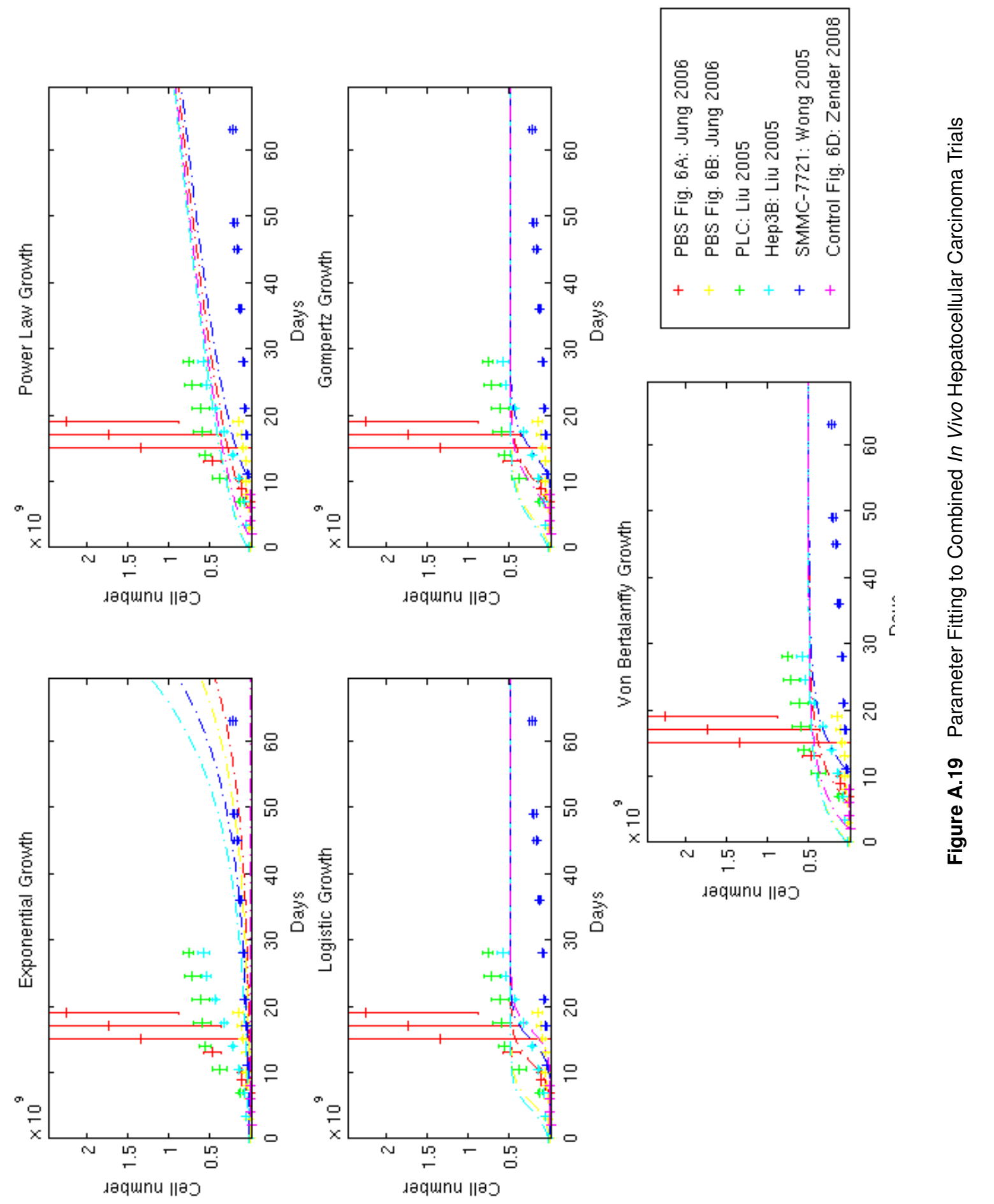



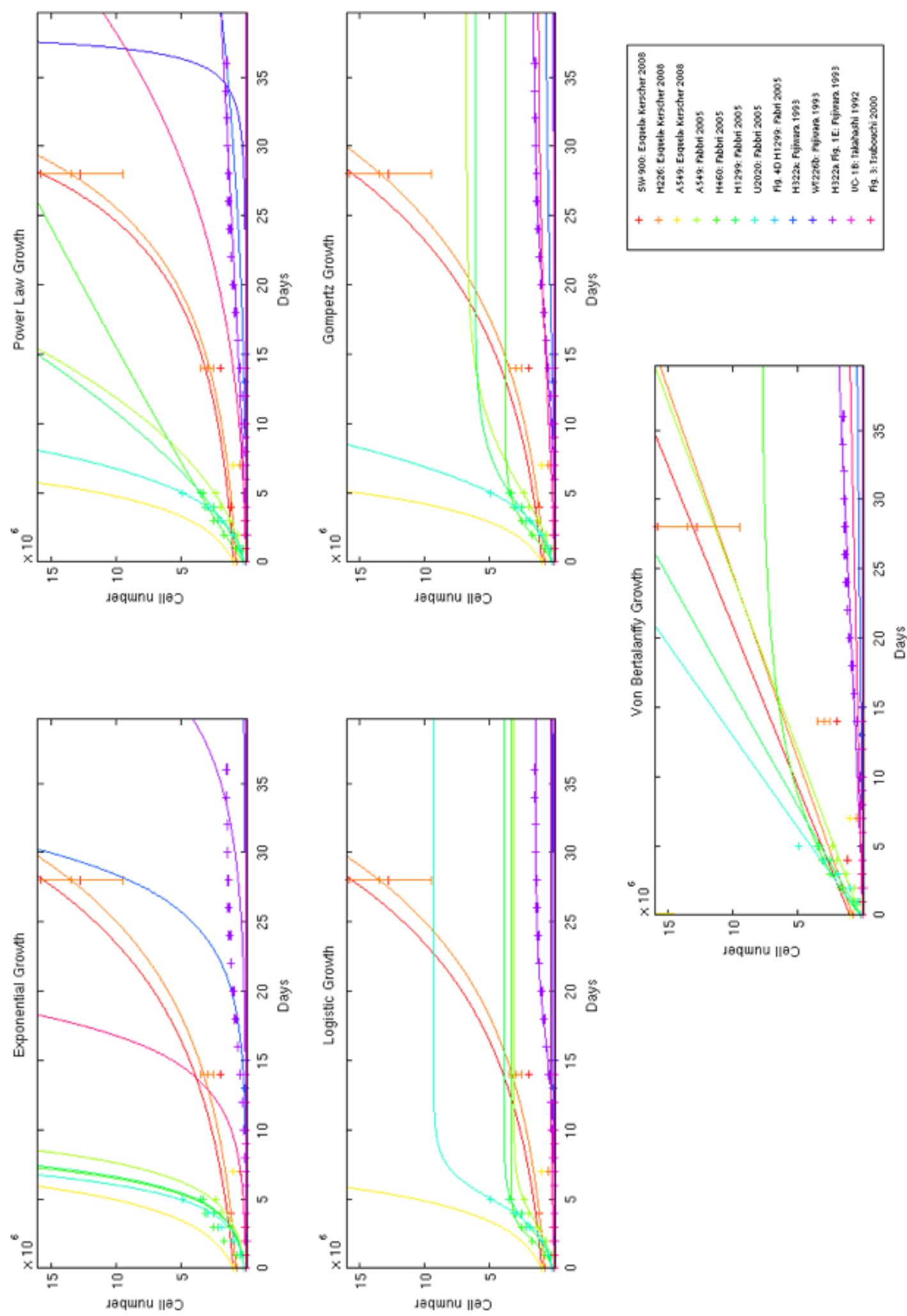

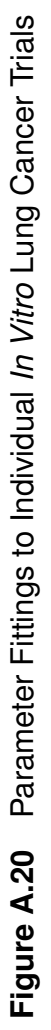



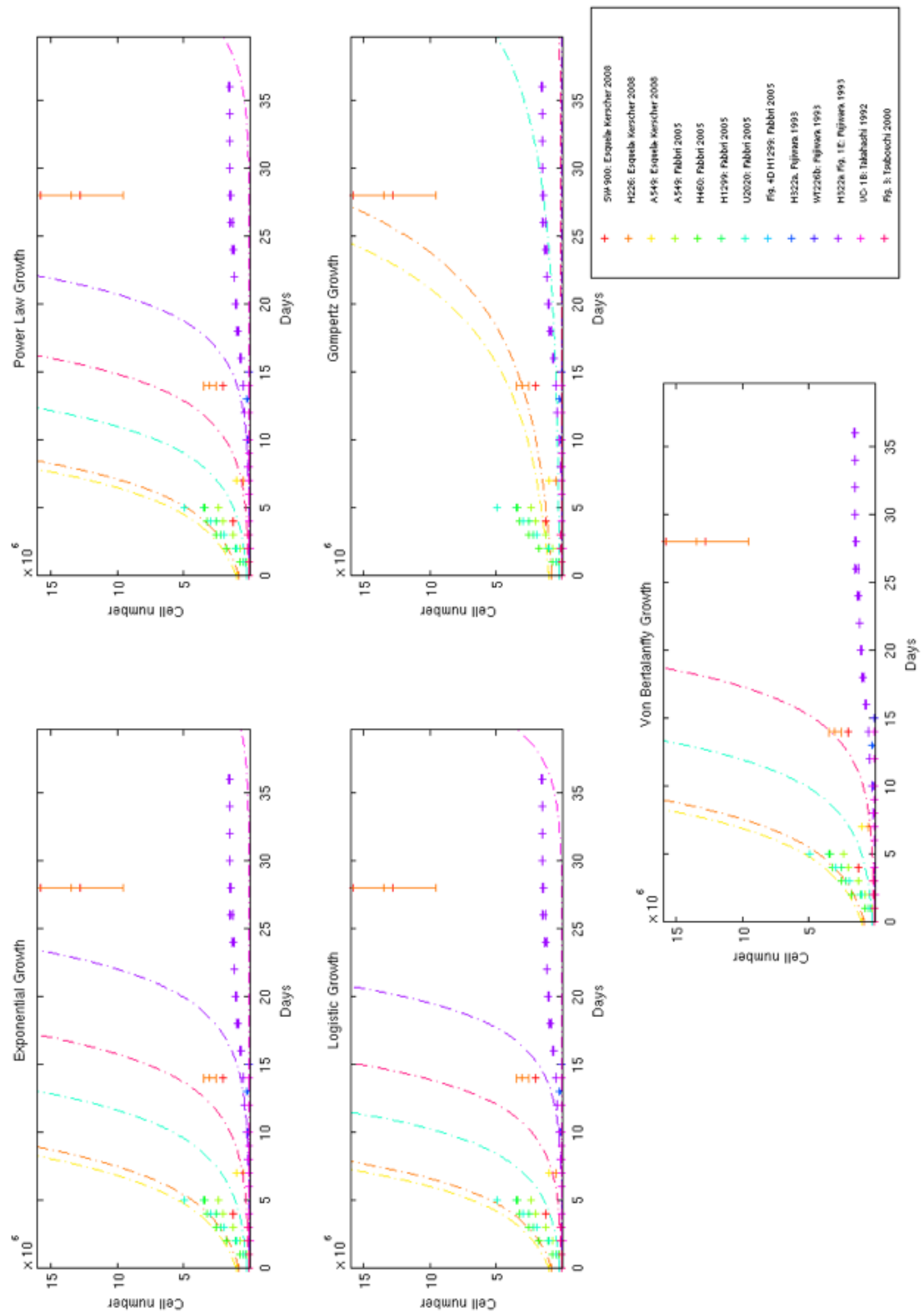


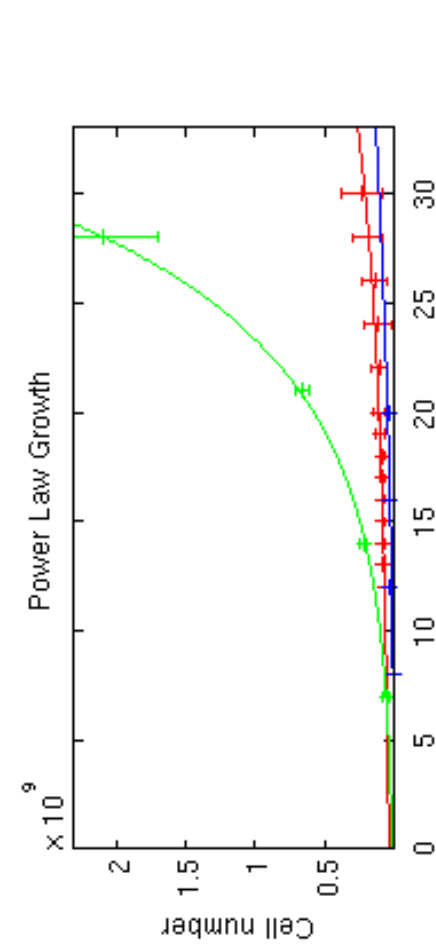

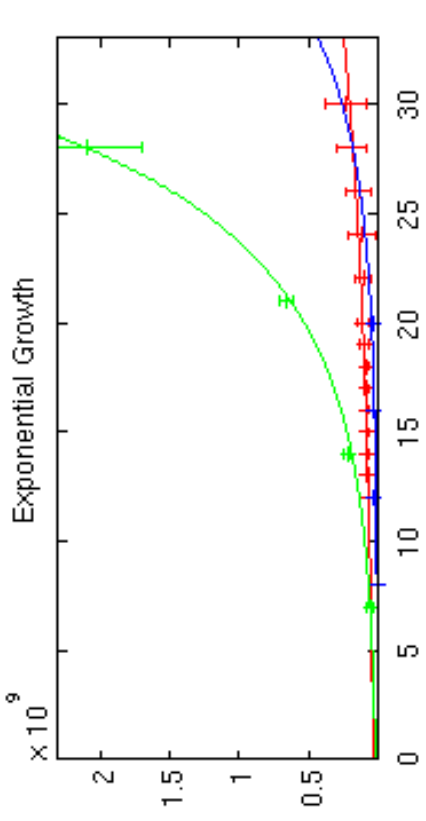

daqunu ॥aว
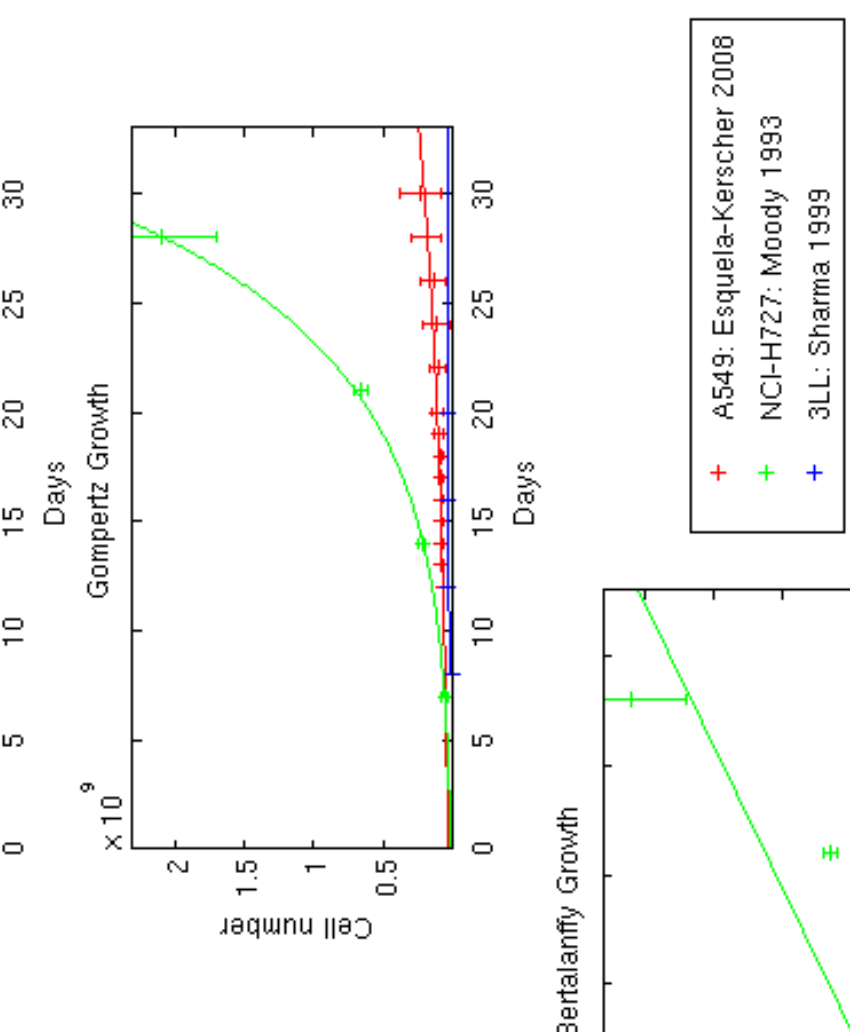

政
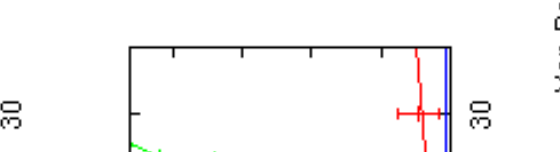

恕
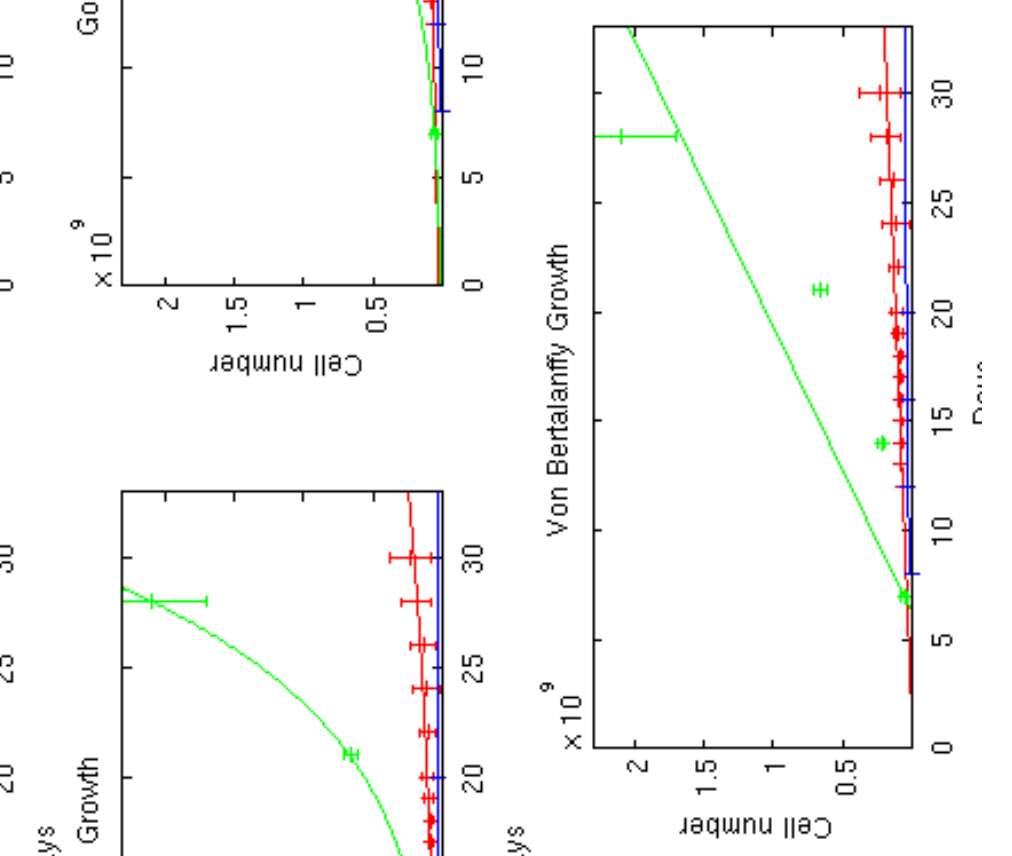

$\stackrel{\square}{N}$

เ

문 


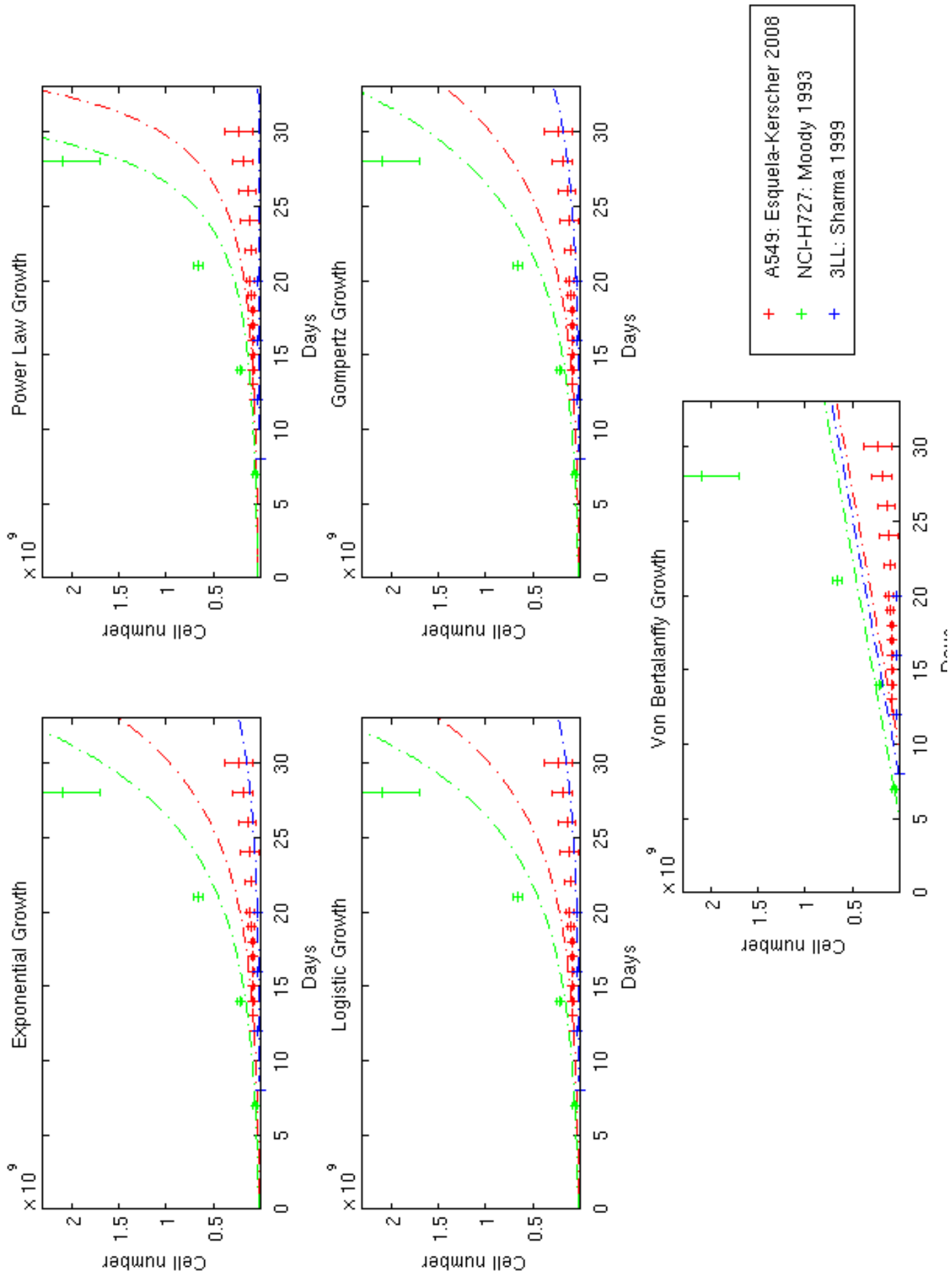



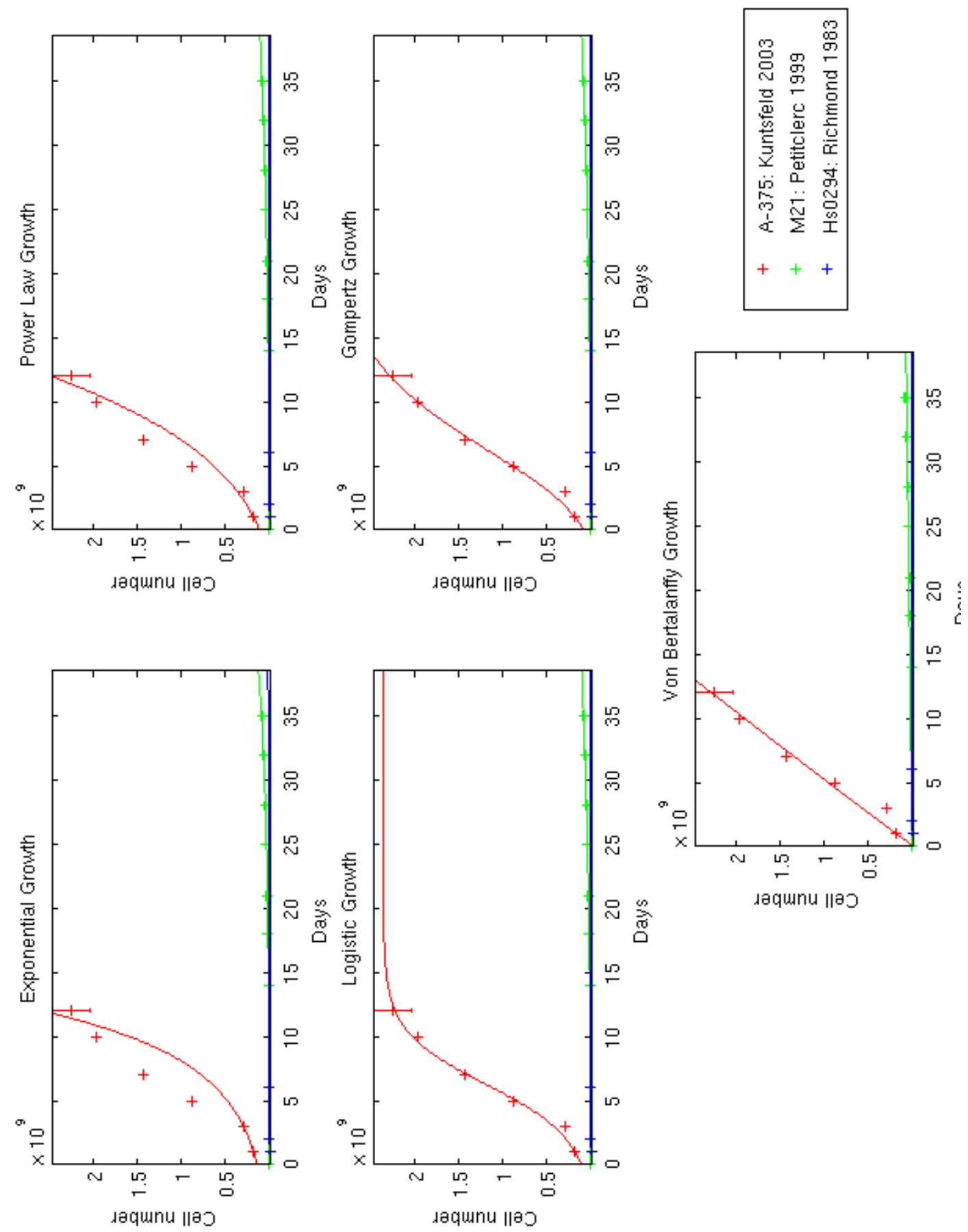

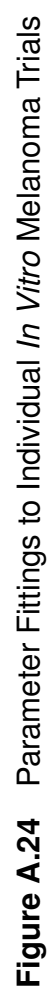



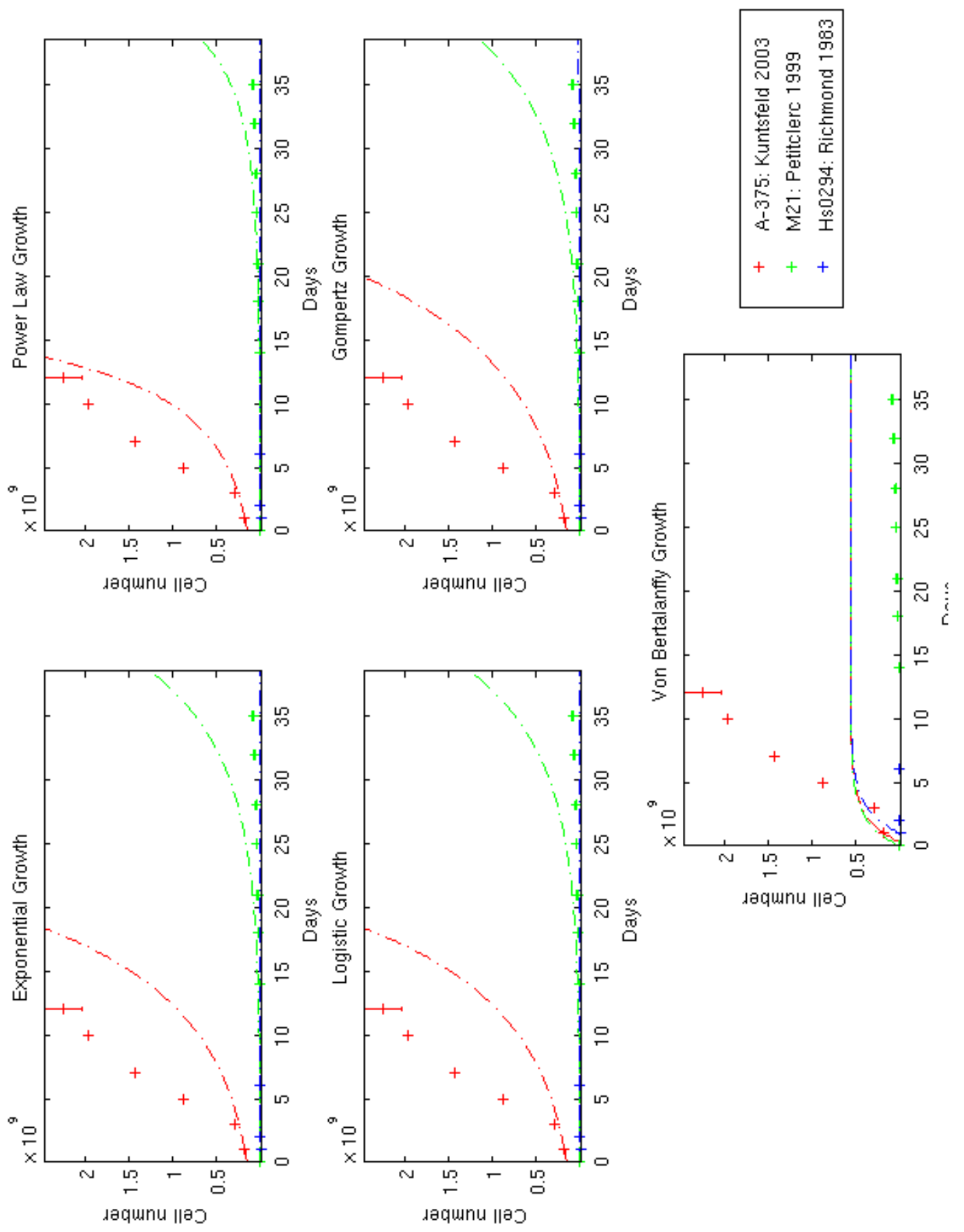

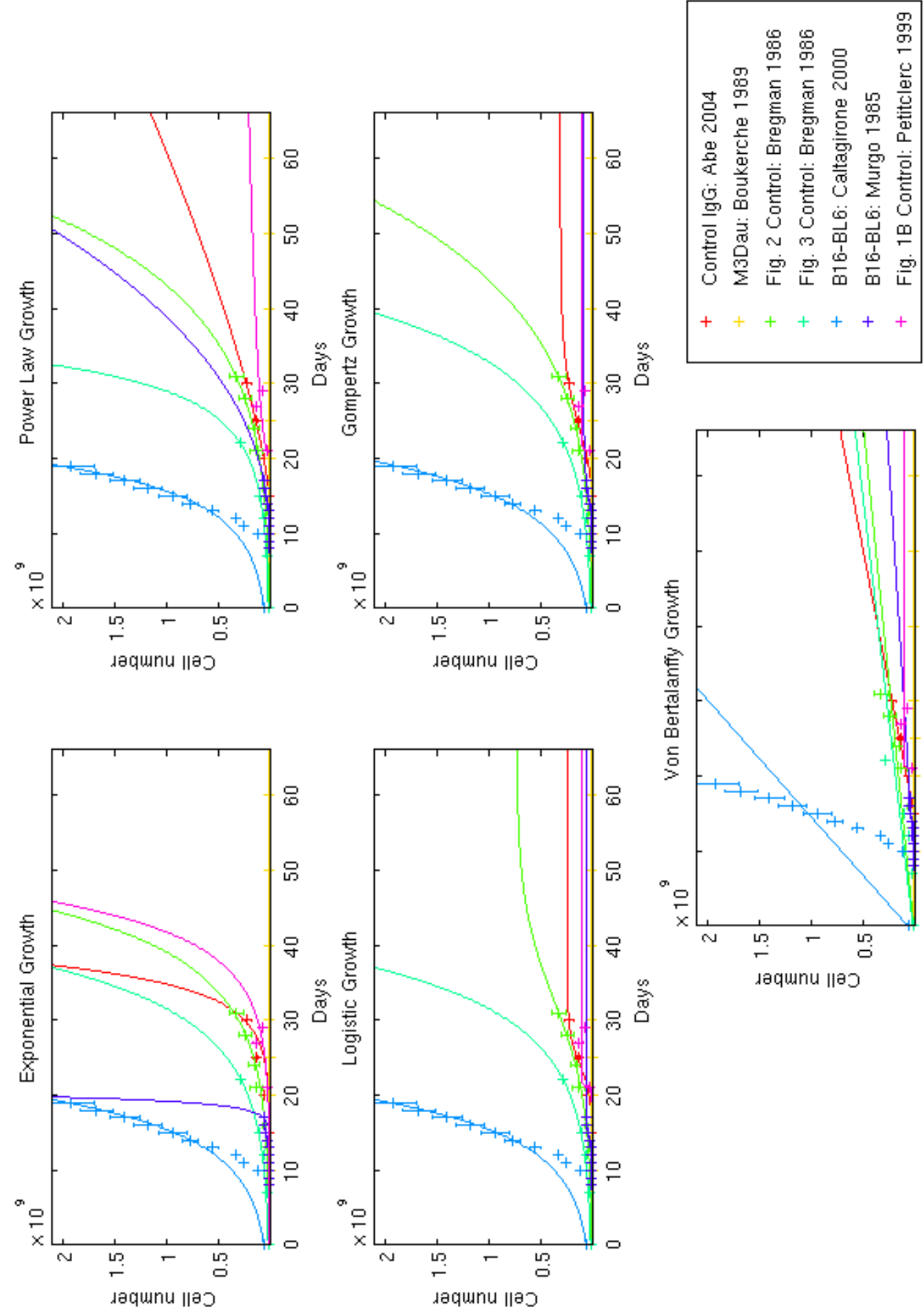

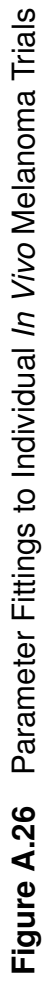




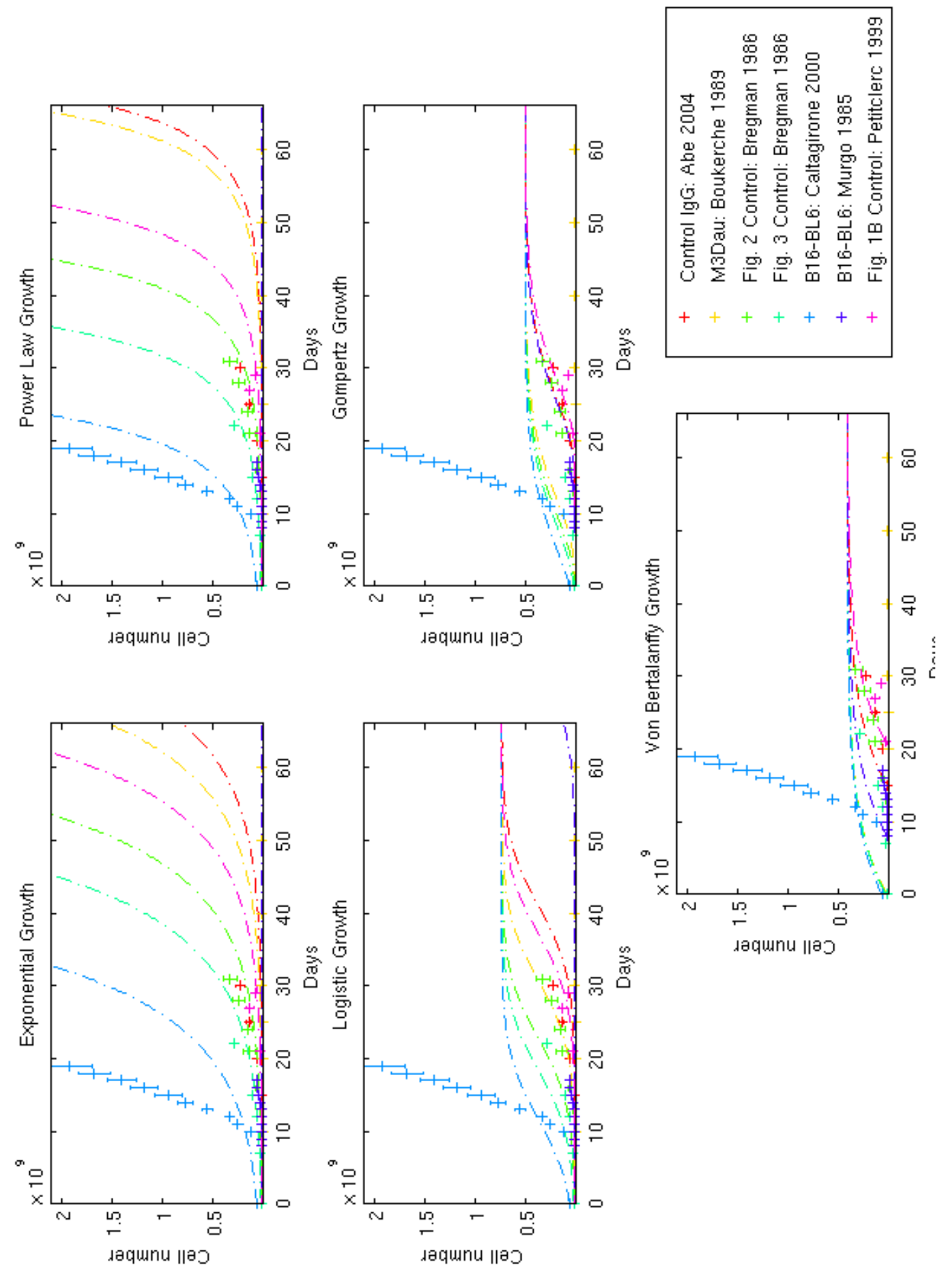



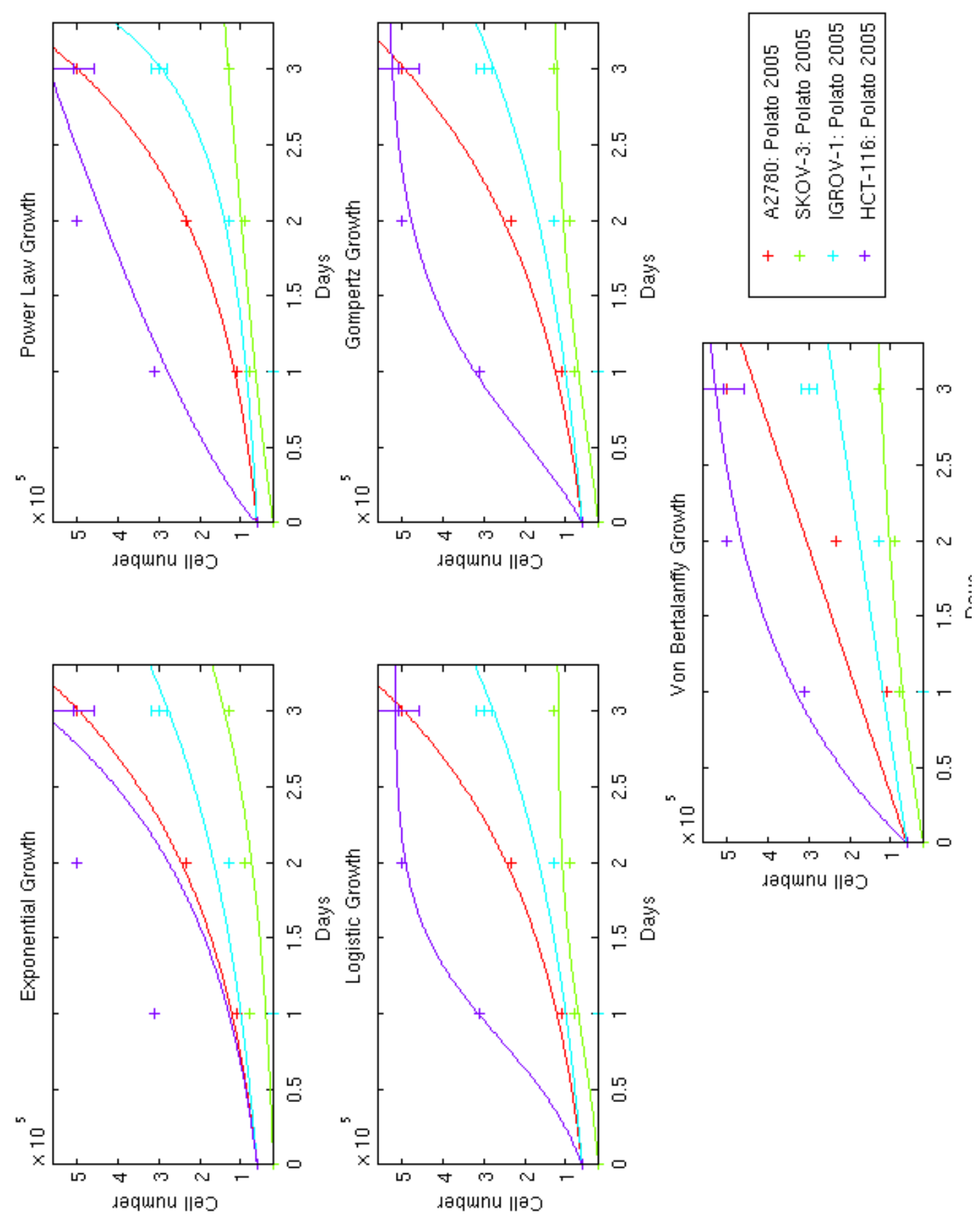


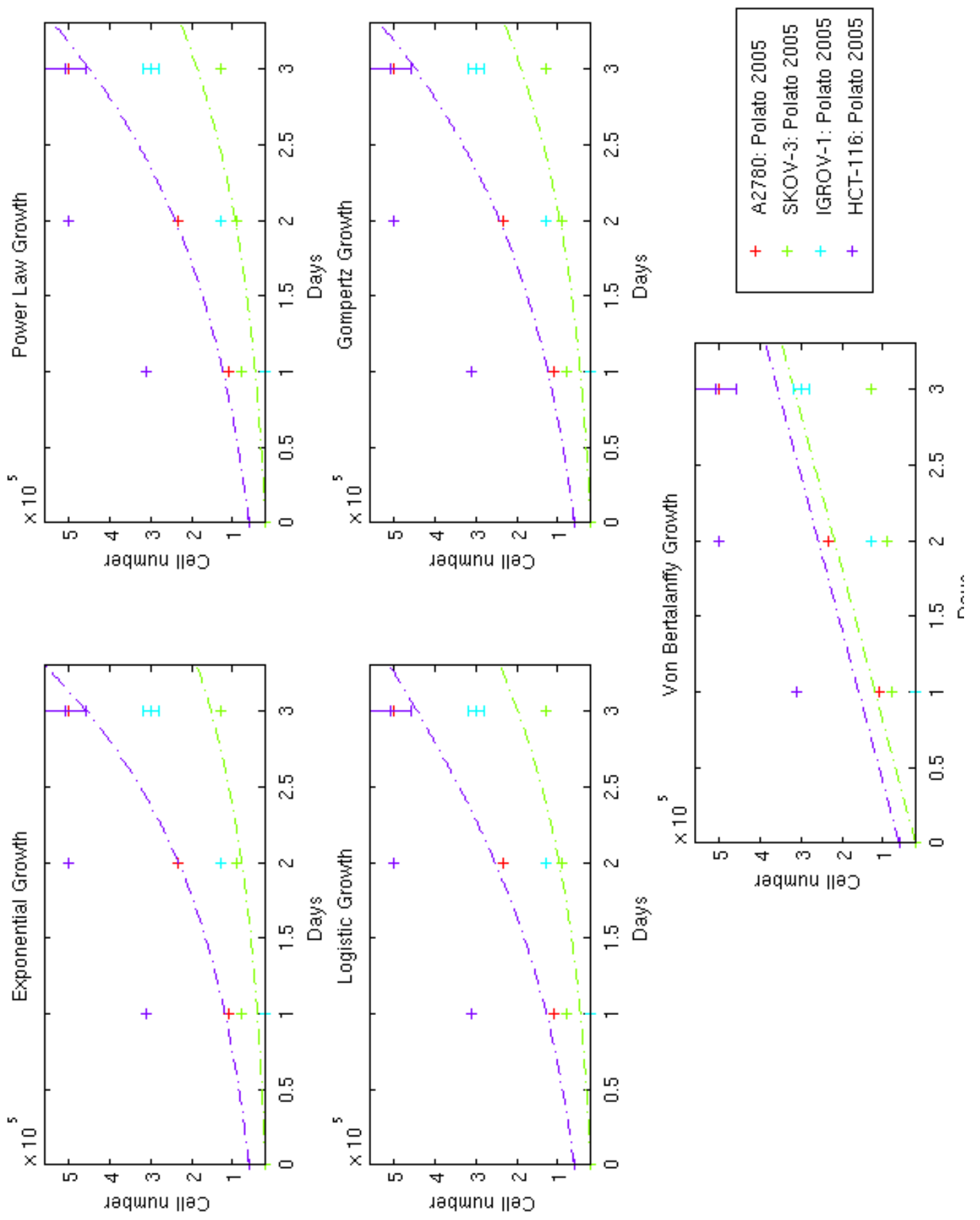



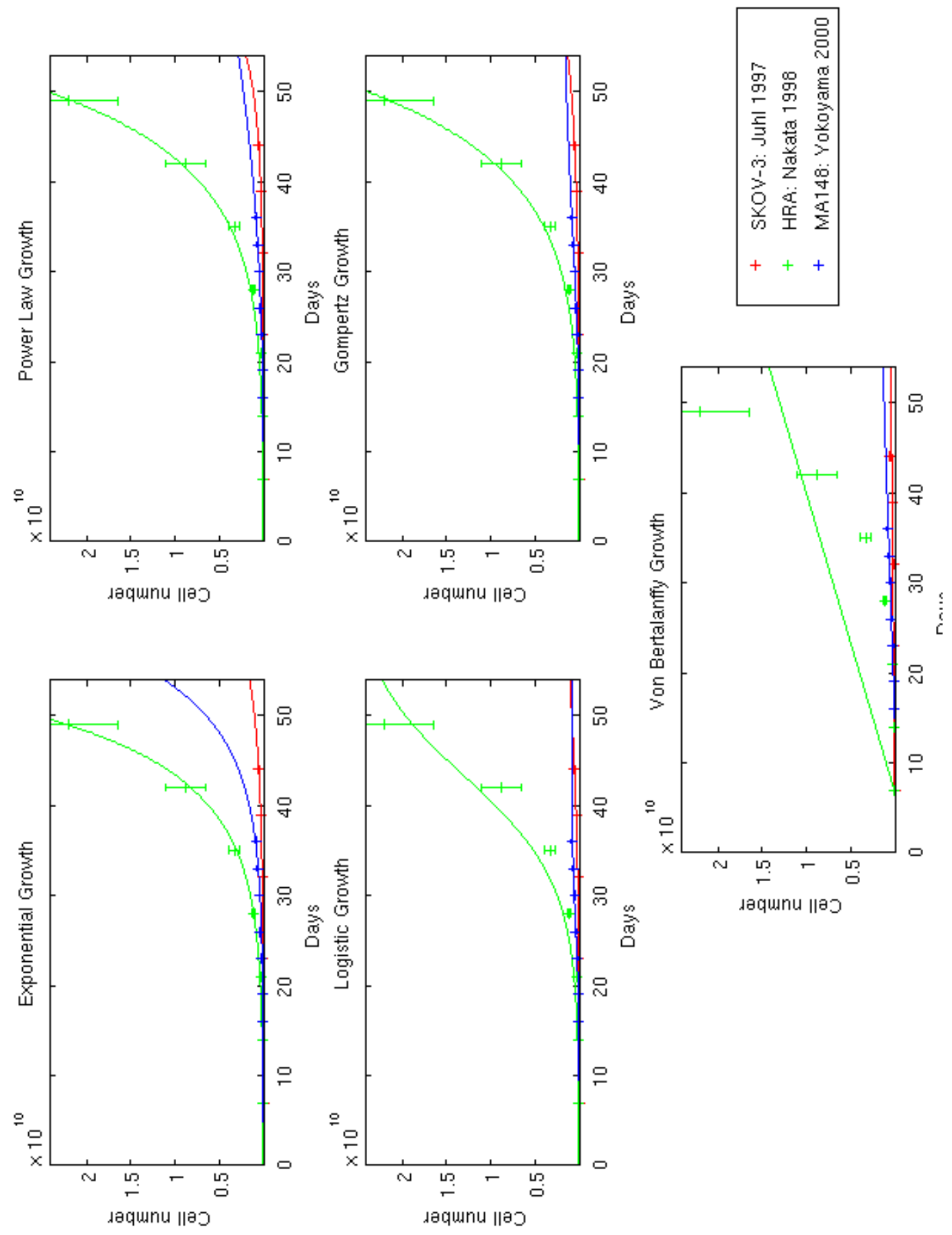


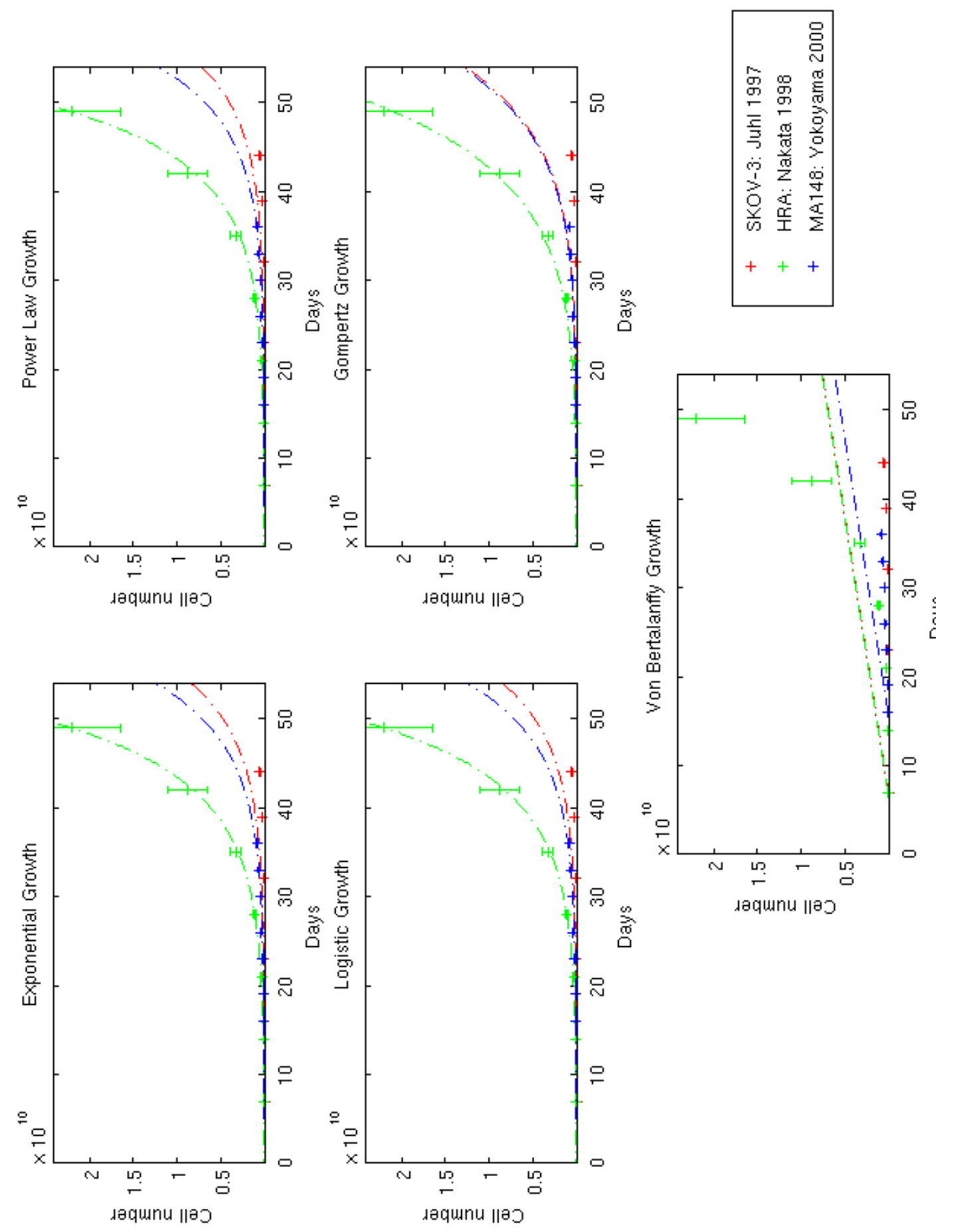



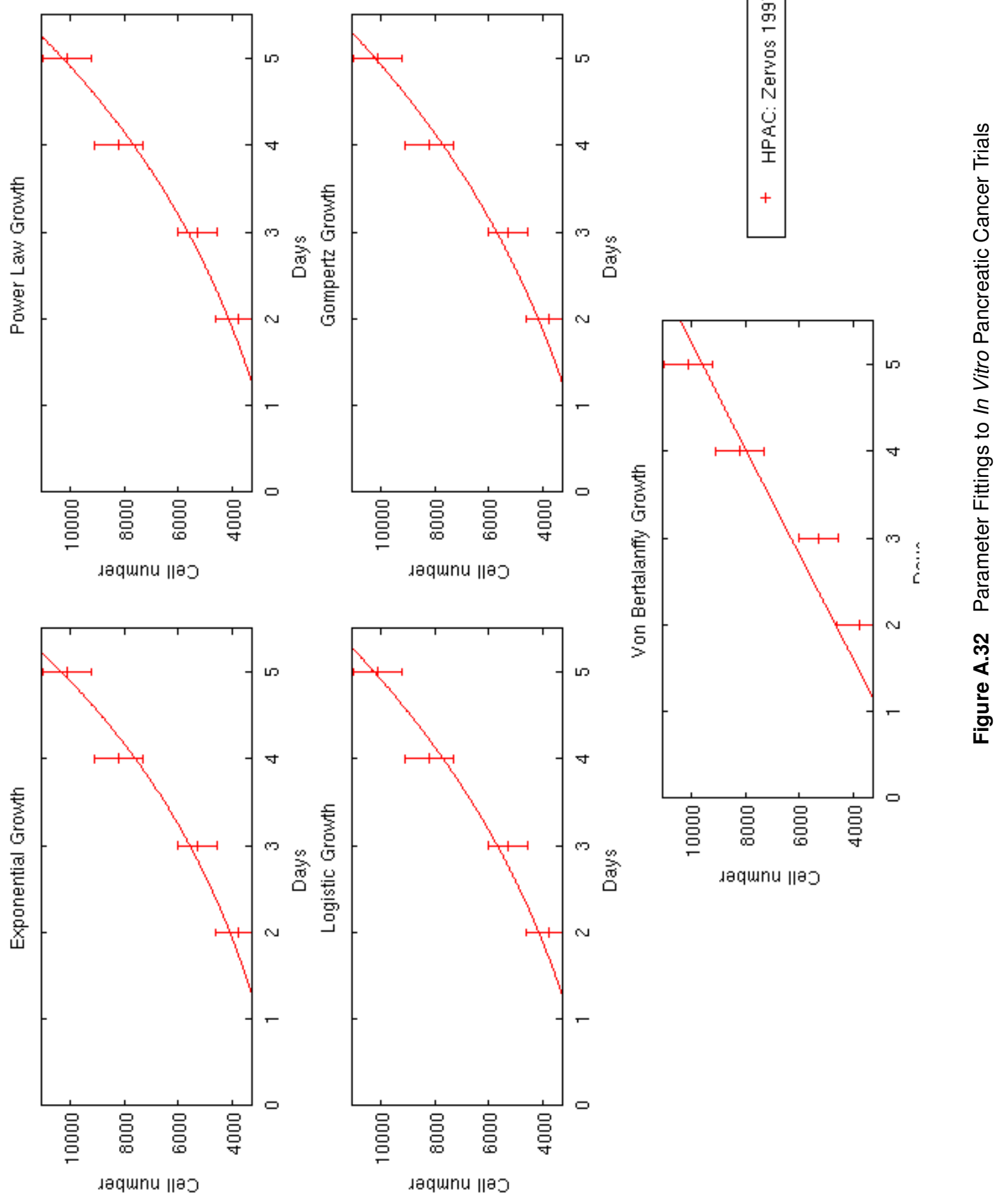

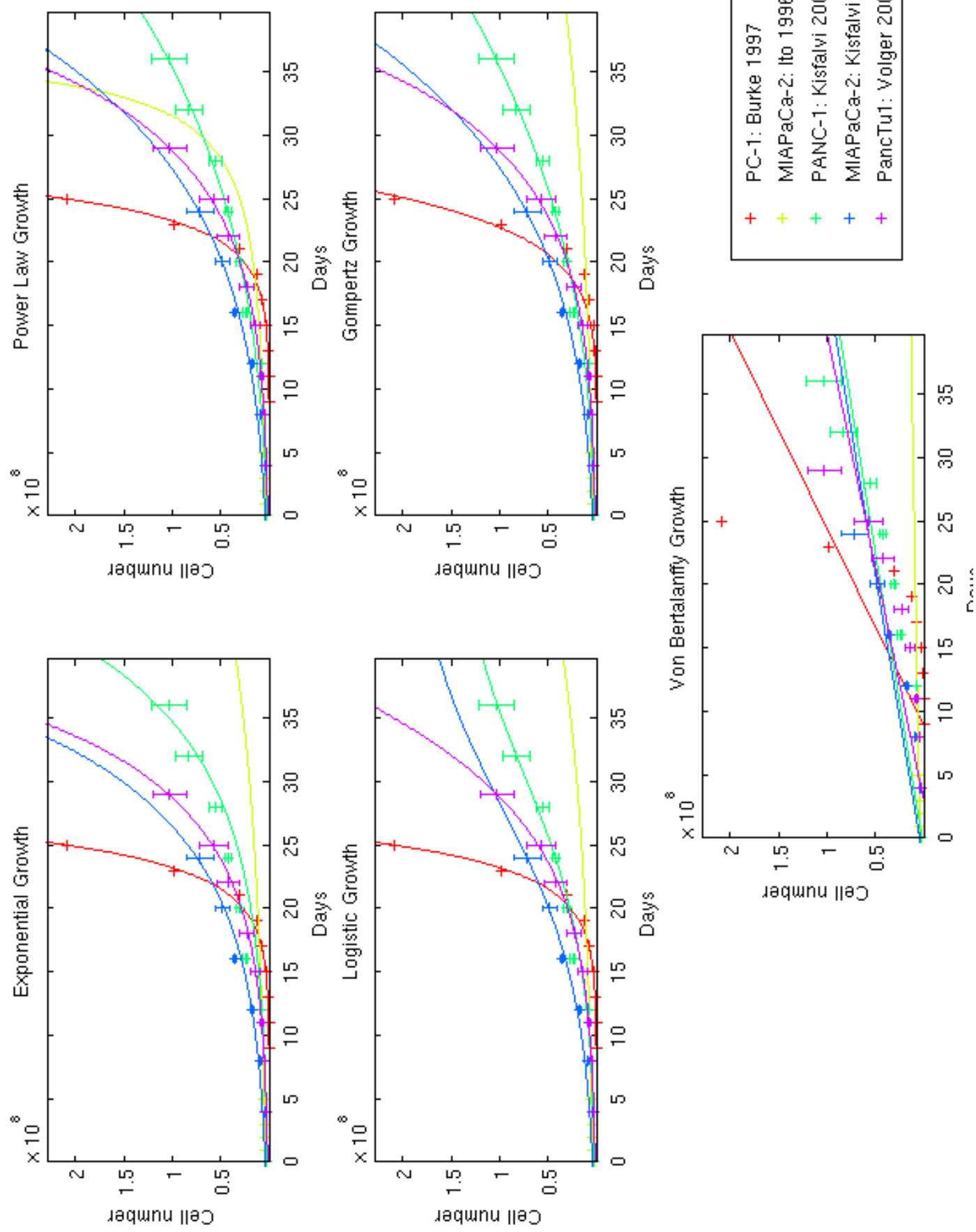

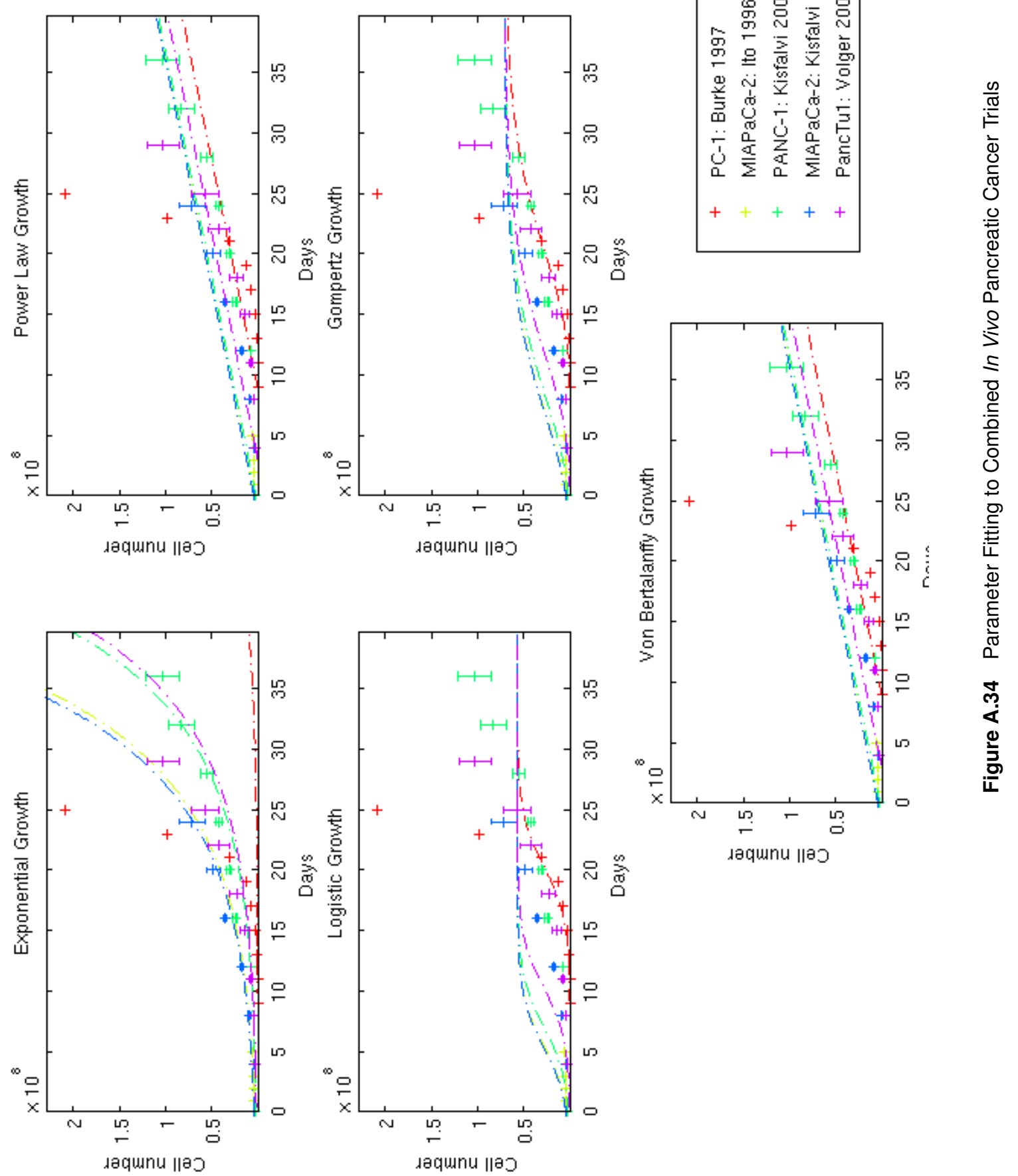

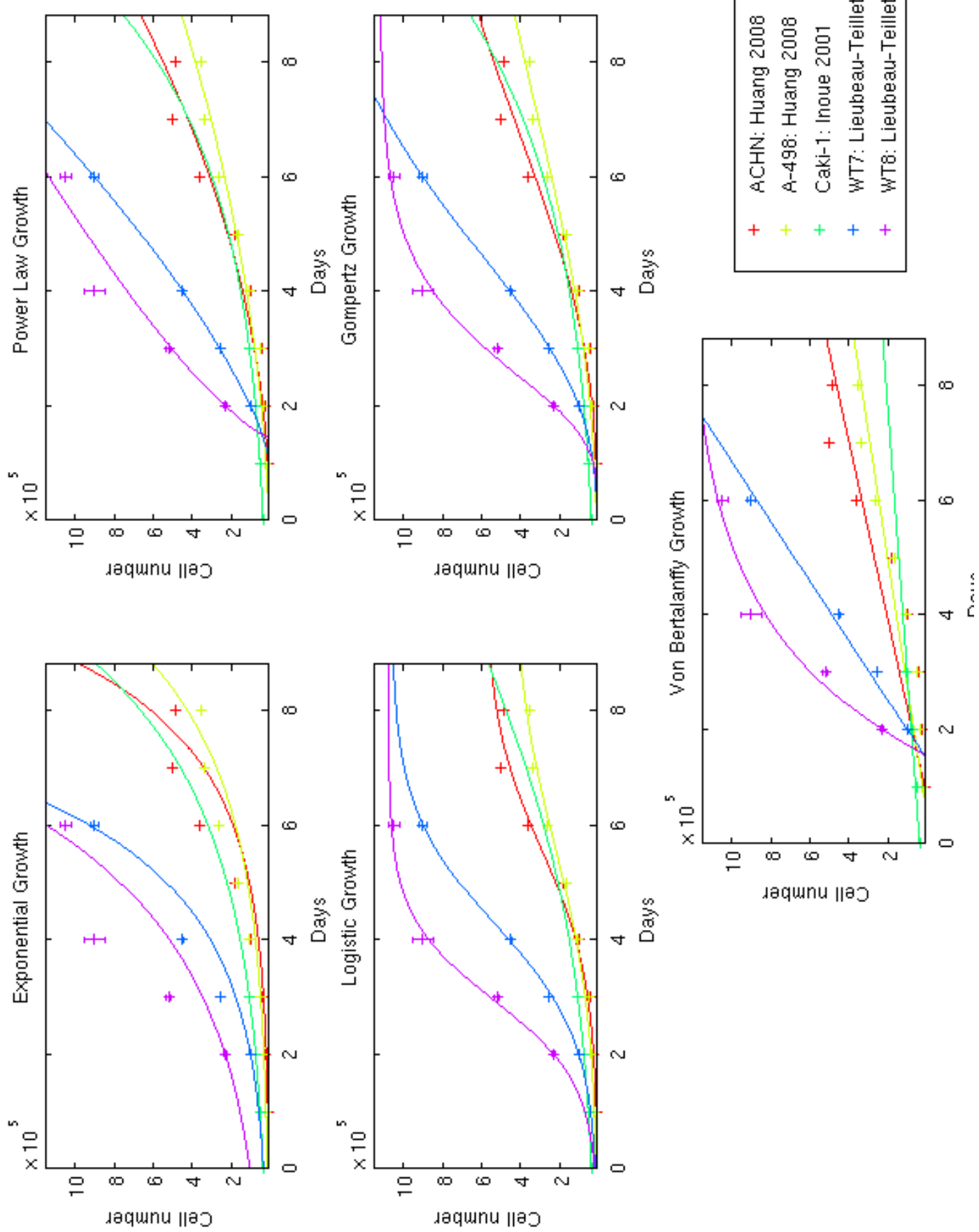

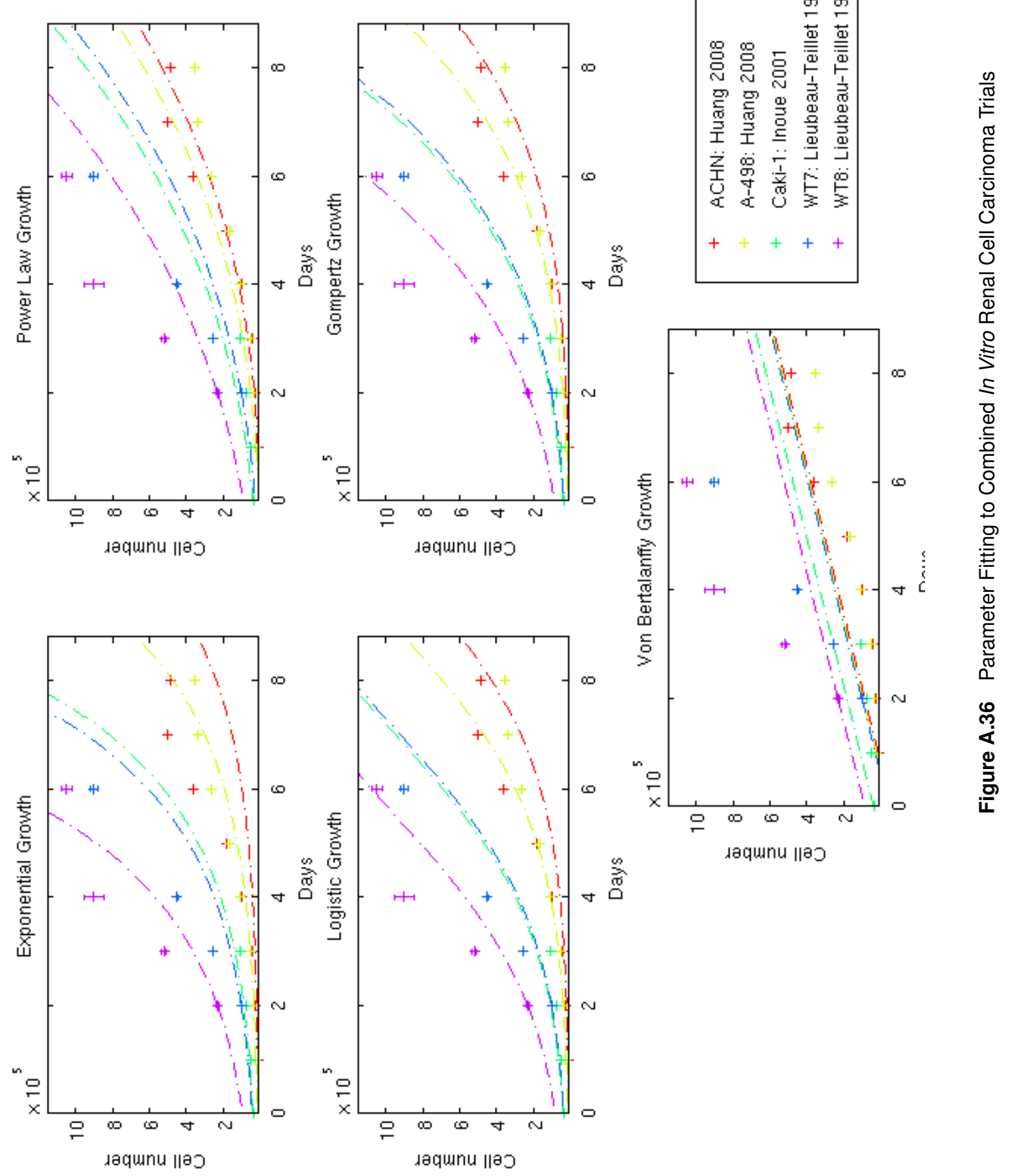


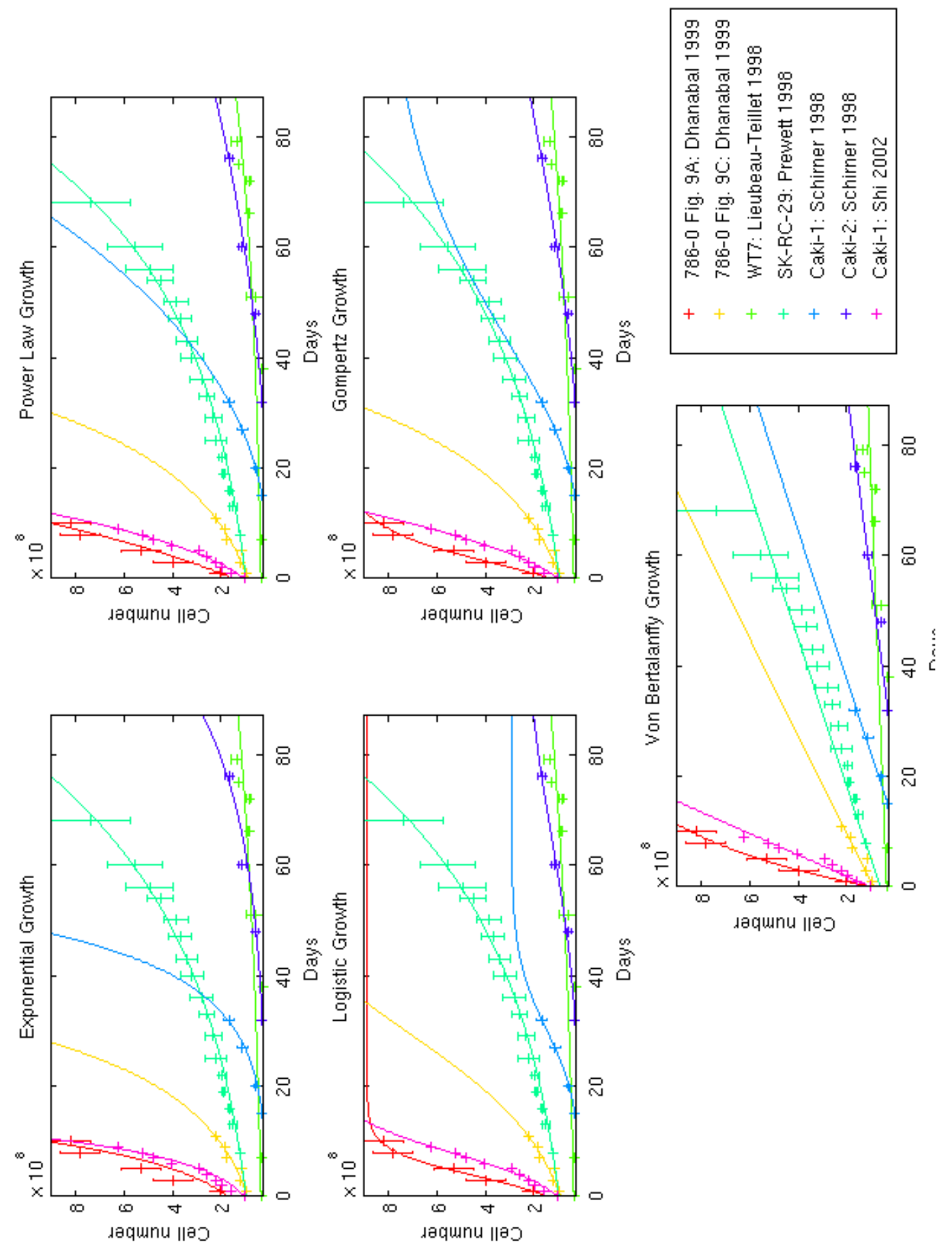

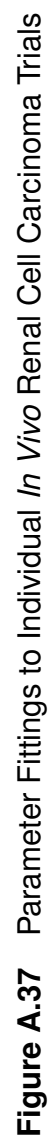




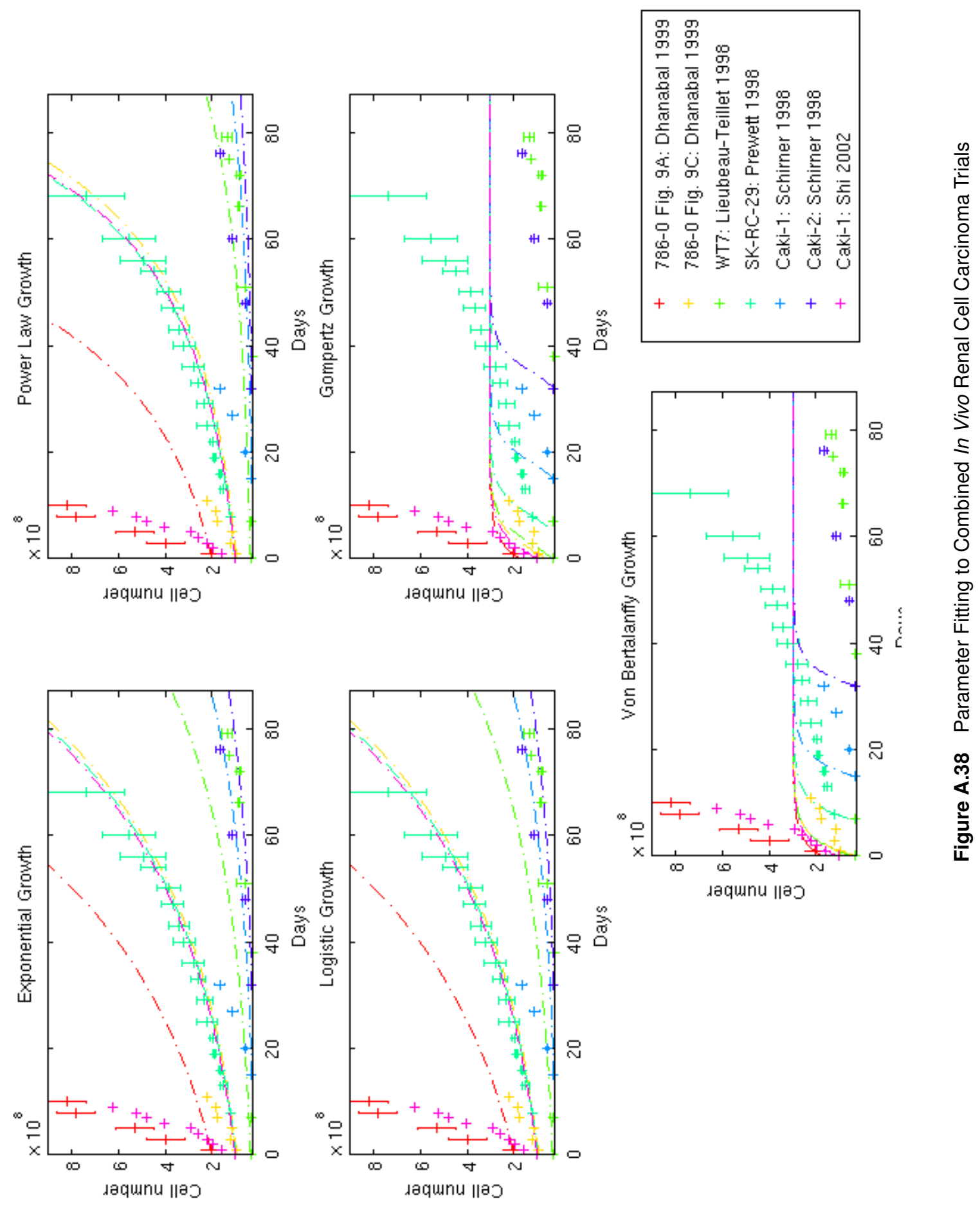





\section{Bibliography}

Abe, Riichiro, Tadamichi Shimizu, Hiroshi Sugawara, Hirokazu Watanabe, Hideki Nakamura, Hiroshi Choei, Nobuyuki Sasaki, Sho-ichi Yamagishi, Masayoshi Takeuchi, and Hiroshi Shimizu. 2004. Regulation of Human Melanoma Growth and Metastasis by AGE-AGE Receptor Interactions. Journal of Investigative Dermatology 122:461-467.

Ahonen, Merja H., Ya-Hua Zhuang, Risto Aine, Timo Ylikomi, and Pentti Tuohimaa. 2000. Androgen receptor and vitamin D receptor in human ovarian cancer: Growth stimulation and inhibition by ligands. International Journal of Cancer 86:40-46.

Aroesty, J., T. Lincoln, N. Shapiro, and G. Boccia. 1973. Tumor growth and chemotherapy: Mathematical methods, computer simulations, and experimental foundations. Mathematical Biosciences 17:243-300.

Ashyraliyev, Maksat, Yves Fomekong-Nanfack, Jaap A. Kaandorp, and Joke G. Joke G. Blom. 2009. Systems biology: parameter estimation for biochemical models. FEBS Journal 276:886-902.

B. Edris et al. 2012. Antibody therapy targeting the CD47 protein is effective in a model of aggressive metastatic leiomyosarcoma. PNAS 17:66566661.

Bastogne, T., A. Samson, P. Vallois, S. Wantz-MŐziŔres, S. Pinel, D. Bechet, and M. Barberi-Heyob. 2010. Phenomenological modeling of tumor diameter growth based on a mixed effects model. Journal of Theoretical Biology 262:544-552.

Belizério, José E. 2009. Immunodeficient Mouse Models: An Overview. The Open Immunology Journal 2.

Boukerche, H, O Berthier-Vergnes, M Bailly, JF Dore, LL Leung, and JL McGregor. 1989. A monoclonal antibody (LYP18) directed against the blood 
platelet glycoprotein IIb/IIIa complex inhibits human melanoma growth in vivo. Blood 74:909-912.

Bregman, Marvin D., Carole Funk, and Masanori Fukushima. 1986. Inhibition of Human Melanoma Growth by Prostaglandin A, D, and J Analogues. Cancer Research 46:2740-2744.

Brooks, Steve, Andrew Gelman, Galin L. Jones, and Xiao-Li Meng. 2011. Handbook of Markov Chain Monte Carlo. Chapman \& Hall/CRC, 1st ed.

Burke, Yvette D., M. Jennifer Stark, Steven L. Roach, Stephanie E. Sen, and Pamela L. Crowell. 1997. Inhibition of Pancreatic Cancer Growth by the Dietary Isoprenoids Farnesol and Geraniol. Lipids 32(2):151-156.

Byrne, H.M., T Alarcon, M.R. Owen, S.D. Webb, and P.K. Maini. 2006. Modelling aspects of cancer dynamics: a review. Phil Trans $R$ Soc 10.

Byrne, H.M. and Cox, S.M. and Kelly, C.E. 2004. Macrophage-tumor interactions: in vivo dynamics. Discrete and Continuous Dynamical Systems $4(1): 81-98$.

Caltagirone, Sara, Cosmo Rossi, Andreina Poggi, Franco O. Ranelletti, Pier Giorgio Natali, Mauro Brunetti, Francesca B. Aiello, and Mauro Piantelli. 2000. Flavonoids apigenin and quercetin inhibit melanoma growth and metastatic potential. International Journal of Cancer 87:595-600.

Chen, Z. Z., S. Y. Zhang, Q. S. Liu, P. F. Xiao, X. Y. Guo, and Z. H. Lu. 2005. The CD47-signal regulatory protein alpha $(\operatorname{SIRP} \alpha)$ interaction is a therapeutic target for human solid tumors. In Proceedings of the 2005 IEEE, 7497-7500. IEEE.

Coopman, Peter J. P., Michael T. H. Do, Mara Barth, Emma T. Bowden, Andrew J. Hayes, Eugenia Basyuk, Jan K. Blancatok, Phyllis R. Vezza, Sandra W. McLeskey, Paul H. Mangeat, and Susette C. Mueller. 2000. The Syk tyrosine kinase suppresses malignant growth of human breast cancer cells. Nature 406:742-747.

de Pillis, L.G., and A.E. Radunskaya. 2006. Some Promising Approaches to Tumor-Immune Modeling. Contemporary Mathematics 410:89-105.

de Pillis, Lisette, Trevor Caldwell, Elizabeth Sarapata, and Heather Williams. 2013a. Mathematical Modeling of Regulatory T Cell Effects on Renal Cell Carcinoma Treatment. AIMS 18(4):915-943. 
de Pillis, Lisette, Renee Fister, , Weiqing Gu, Craig Collins, Michael Daub, David Gross, James Moore, and Benjamin Preskill. 2009. Mathematical Model Creation for Cancer Chemo-Immunotherapy. Computational and Mathematical Methods in Medicine 10(3):165-184.

de Pillis, Lisette, Angela Gallegos, and Ami Radunskaya. 2013b. A Model of Dendritic Cell Therapy for Melanoma. Frontiers in Molecular and Cellular Oncology 3.

de Pillis, Lisette, Ami Radunskaya, and Charles Wiseman. 2005. A Validated Mathematical Model of Cell-Mediated Immune Response to Tumor Growth. Journal of Cancer Research 65:7950-7958.

Dennis, John E. Jr, and Daniel J. Woods. 1987. New Computing Environments: Microcomputers in Large-Scale Scientific Computing, chap. Optimization on Microcomputers: The Nelder-Mead Simplex Algorithm. SIAM.

Dhanabal, Mohanraj, Ramani Ramchandran, and Ruediger Volk. 1999. Endostatin : Yeast Production, Mutants, and Antitumor Effect in Renal Cell Carcinoma. Cancer Research 59:189-197.

Du, Zhijun, and Shukun Hou. 2003. The Anti-Angiogenic Activity of Human Endostatin Inhibits Bladder Cancer Growth and Its Mechanism. The Journal of Urology 170:2000-2003.

Duffey, Dianne C., Zhong Chen, and Gang Dong. 1999. Expression of a Dominant-Negative Mutant Inhibitor- $\kappa \mathrm{B} \alpha$ of Nuclear Factor- $\kappa \mathrm{B}$ in $\mathrm{Hu}-$ man Head and Neck Squamous Cell Carcinoma Inhibits Survival, Proinflammatory Cytokine Expression, and Tumor Growth in Vivo. Cancer Research 59:3468-3474.

Elishmereni, Moran, Yuri Kheifetz, Henrik Söndergaard, Rune Viig Overgaard, and Zvia Agur. 2011. An Integrated Disease/Pharmacokinetic/Pharmacodynamic Model Suggests Improved Interleukin-21 Regimens Validated Prospectively for Mouse Solid Cancers. PLoS Computational Biology 7.

Esquela-Kerscher, Aurora, Phong Trang, Jason F. Wiggins, Lubna Patrawala, Angie Cheng, Lance Ford, Joanne B. Weidhaas, David Brown, Andreas G. Bader, and Frank J. Slack. 2008. The let-7 microRNA reduces tumor growth in mouse models of lung cancer. Cell Cycle 7:759-764. 
Fabbri, Muller, Dimitrios Iliopoulos, Francesco Trapasso, Rami I. Aqeilan, Amelia Cimmino, Nicola Zanesi, Sai Yendamuri, Shuang-Yin Han, Dino Amadori, Kay Huebner, and Carlo M. Croce. 2005. WWOX gene restoration prevents lung cancer growth in vitro and in vivo. PNAS 102:15,61115,616 .

Fujimoto, Naohiro, Atsuo Sugita, Yoshio Terasawa, and Masakazu Kato. 1995. Observations on the Growth Rate of Renal Cell Carcinoma. International Journal of Urology 2:71-76.

Fujiwara, Toshiyoshi, Elizabeth A. Grimm, and Tapas Mukhopadhyay. 1993. A Retroviral Wild-type p53 Expression Vector Penetrates Human Lung Cancer Spheroids and Inhibits Growth by Inducing Apoptosis. Cancer Research 53:4129-4133.

Gilks, W. Walter R., Sylvia Richardson, and David J. Spiegelhalter. 1996. Markov Chain Monte Carlo in Practice: Interdisciplinary Statistics. Chapman \& Hall/CRC, 1st ed.

Golshani, Roozbeh, Luis Lopez, and Veronica Estrella. 2008. Hyaluronic Acid Synthase-1 Expression Regulates Bladder Cancer Growth, Invasion, and Angiogenesis through CD44. Cancer Research 68:483-491.

Gomero, Boloye. 2012. Latin Hypercube Sampling and Partial Rank Correlation Coefficient Analysis Applied to an Optimal Control Problem. Master's Thesis, University of Tennessee.

Guiot, Caterina, Piero Giorgio Degiorgis, Pier Paolo Delsanto, Pietro Gabriele, and Thomas S. Deisboeck. 2003. Does tumor growth follow a "universal law"? Journal of Theoretical Biology 225:147-151.

Hart, D, E Shochat, and Z Agur. 1998. The growth law of primary breast cancer as inferred from mammography screening trials data. British Journal of Cancer 78:382-387.

Heuser, Louis, John S. Spratt, and Hiram C Jr. Polk. 1979. Growth Rates of Primary Breast Cancers. Cancer 43:1888-1894.

Huang, Dan, Yan Ding, and Wang-Mei Luo. 2008. Inhibition of MAPK Kinase Signaling Pathways Suppressed Renal Cell Carcinoma Growth and Angiogenesis In vivo. Cancer Research 68:81-88. 
Huynh, Hung, Pierce K.H. Chow, Nallasivam Palanisamy, and Manuel Salto-Tellez. 2008. Bevacizumab and rapamycin induce growth suppression in mouse models of hepatocellular carcinoma. Journal of Hepatology 49:52-60.

Inoue, Ken-ichiro, Yutaka Kawahito, Yasunori Tsubouchi, Masataka Kohno, Rikio Yoshimura, Toshikazu Yoshikawa, and Hajime Sano. 2001. Expression of Peroxisome Proliferator-Activated Receptor $\gamma$ in Renal Cell Carcinoma and Growth Inhibition by Its Agonists. Biochemical and Biophysical Research Communications 287:727-732.

Ito, Toshio, Sumio Kawata, Shinji Tamura, Takumi Igura, Toshihiko Nagase, Jun-ichiro Miyagawa, Eiji Yamazaki, Hiroshi Ishiguro, and Yuji Matsuzawa. 1996. Suppression of Human Pancreatic Cancer Growth in BALB/c Nude Mice by Manumycin, a Farnesyl:protein Transferase Inhibitor. Cancer Science 87(2):113-116.

Jackson, Trachette L., and Helen M. Byrne. 2000. A mathematical model to study the effects of drug resistance and vasculature on the response of solid tumors to chemotherapy. Mathematical Biosciences 164:17-38.

Jane E. Visvader. 2011. Cells of Origin in Cancer. Nature 469:314-322.

Juhl, Hartmut, Sean G. Downing, Anton Wellstein, and Frank Czubayko. 1997. HER-2/neu Is Rate-limiting for Ovarian Cancer Growth. Journal of Biological Chemistry 272(47):29,482-29,486.

Jung, Cho-Rok, Jinsang Yoo, Ye Jin Jang, Sangsoo Kim, In-Sun Chu, Young Il Yeom, Jong Young Choi, and Dong-Soo Im. 2006. AdenovirusMediated Transfer of siRNA Against PTTG1 Inhibits Liver Cancer Cell Growth In Vitro and In Vivo. Journal of Hepatology 43:1042-1052.

Kamada, Masayuki, Alan So, and Mototsugu Muramaki. 2007. Hsp27 knockdown using nucleotide-based therapies inhibit tumor growth and enhance chemotherapy in human bladder cancer cells. Molecular Cancer Therapeutics 6:299-308.

Kisfalvi, Krisztina, Guido Eibl, and James Sinnett-Smith. 2009. Metformin Disrupts Crosstalk between G ProteinấĽCoupled Receptor and Insulin Receptor Signaling Systems and Inhibits Pancreatic Cancer Growth. Cancer Research 69:6539-6545. 
Koch, Ulrike, Mechthild Krause, and Michael Baumann. 2010. Cancer stem cells at the crossroads of current cancer therapy failuresï $£_{i}$ a Radiation oncology perspective. Seminars in Cancer Biology 20.

Kunstfeld, Rainer, Georg Wickenhauser, Uwe Michaelis, Michael Teifel, Wolfgang Umek, Kurt Naujoks, Klaus Wolsh, and Peter Petzelbauer. 2003. Paclitaxel Encapsulated in Cationic Liposomes Diminishes Tumor Angio-

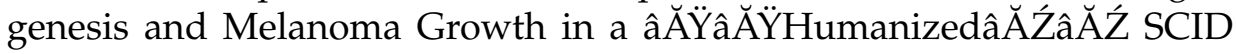
Mouse Model. Journal of Investigative Dermatology 120:476-482.

Lagarias, Jeffrey C., James A. Reed, Margaret H. Wright, and Paul E. Wright. 1998. Convergence Properties of the Nelder-Mead Simplex Method in Low Dimensions. SIAM Journal on Optimization 9:112-147.

Lieubeau-Teillet, Blandine, Janusz Rak, and Serge Jothy. 1998. von HippelLindau Gene-mediated Growth Suppression and Induction of Differentiation in Renal Cell Carcinoma Cells Grown as Multicellular Tumor Spheroids. Cancer Research 58:4957-4962.

Liu, Ta-Jen, Mary Wang, Randall L. Breau, Ying Henderson, Adel K. ElNaggar, Kimberly D. Steck, Michael W. Sicard, and Gary L. Clayman. 1999. Apoptosis induction by E2F-1 via adenoviral-mediated gene transfer results in growth suppression of head and neck squamous cell carcinoma cell lines. Cancer Gene Therapy 6:163-172.

Liu, Yuqing, Ronnie T. Poon, and Qinyu Li. 2005. Both Antiangiogenesisand Angiogenesis-Independent Effects Are Responsible for Hepatocellular Carcinoma Growth Arrest by Tyrosine Kinase Inhibitor PTK787 / ZK222584. Cancer Research 65:3691-3699.

López, S. et al. 2004. Statistical evaluation of mathematical models for microbial growth. International Journal of Food Microbiology 96:289-300.

LoTempio, Maria M., Mysore S. Veena, and Helen L. Steele. 2005. Curcumin Suppresses Growth of Head and Neck Squamous Cell Carcinoma. Clinical Cancer Research 11:6994-7002.

Lu, Runqing, and Ginette Serrero. 1999. Resveratrol, a Natural Product Derived From Grape, Exhibits Antiestrogenic Activity and Inhibits the Growth of Human Breast Cancer Cells. Journal of Cellular Physiology 179:297-304. 
Martin, Sarah A., Nuala McCabe, Michelle Mullarkey, Robert Cummins, Darren J. Burgess, Yusaku Nakabeppu, Sugako Oka, Elaine Kay, Christopher J. Lord, and Alan Ashworth. 2010. DNA Polymerases as Potential Therapeutic Targets for Cancers Defcient in the DNA Mismatch Repair Proteins MSH2 or MLH1. Cancer Cell 17:235-248.

Miyake, Hideaki, Isao Hara, and Sadao Kamidono. 2001. Synergistic Chemsensitization and Inhibition of Tumor Growth and Metastasis by the Antisense Oligodeoxynucleotide Targeting Clusterin Gene in a $\mathrm{Hu}-$ man Bladder Cancer Model. Clinical Cancer Research 7:4245-4252.

Moody, Terry W., Farah Zia, Muriel Draoui, Douglas E. Brenneman, Mati Fridkin, Ariane Davidson, and Illiana Gozes. 1993. A vasoactive intestinal peptide antagonist inhibits non-small cell lung cancer growth. Biochemistry 90:4345-4349.

Mummert, Anna. 2010. Parameter sensitivity analysis for mathematical modeling. Presented at the Math department research seminar at Marshall University.

Murgo, Anthony J. 1985. Inhibition of B16-BL6 Melanoma Growth in Mice by Methionine-Enkephalin. Journal of the National Cancer Institute 75:476482 .

Nagl, Sylvia, and Manish Patel. 2006. Cancer Bioinformatics: From Therapy Design to Treatment, chap. Mathematical Models of Cancer. John Wiley \& Sons, Ltd, Chichester, UK.

Nakagawa, Hiroyuki, Koji Tsuta, Katsuji Kiuchi, Hideto Senzaki, Kanji Tanaka, Koshiro Hioki, and Airo Tsubura. 2001. Growth inhibitory effects of diallyl disuliňAde on human breast cancer cell lines. Carcinogenesis 22:891-897.

Nakata, Hideyuki, Yoshihiro Kikuchi, Takehiko Tode, Junko Hirata, Tsunekazu Kita, Kenji Ishii, Kazuya Kudoh, Ichiro Nagata, and Nariyoshi Shinomiya. 1998. Inhibitory Effects of Ginsenoside $\mathrm{Rh}_{2}$ on Tumor Growth in Nude Mice Bearing Human Ovarian Cancer Cells. Japanese Journal of Cancer Research 89:733-740.

Norton, Larry. 1988. A Gompertzian Model of Human Breast Cancer Growth. Cancer Research 48:7067-7071. 
Ohnishi, Shunsuke, Sumiko Ohnami, Friedrich Laub, Kazunori Aoki, Koichi Suzuki, Yae Kanai, Kazunori Haga, Masahiro Asaka, Francesco Ramirez, and Teruhiko Yoshida. 2003. Downregulation and growth inhibitory eïn̆ Ăect of epithelial-type Krüppel-like transcription factor KLF4, but not KLF5, in bladder cancer. Biochemical and Biophysical Research Communications 308:251-256.

Okegawa, Takatsugu, Rey-Chen Pong, and Yingming Li. 2001. The Mechanism of the Growth-inhibitory Effect of Coxsackie and Adenovirus Receptor (CAR) on Human Bladder Cancer: A Functional Analysis of CAR Protein Structure. Cancer Research 61:6592-6600.

Paquin, Dana, Peter S. Kim, Peter P. Lee, and Doron Levy. 2010. Strategic Treatment Interruptions During Imatinib Treatment of Chronic Myelogenous Leukemia. Bull Math Biol 73:1082-1100.

Petitclerc, Eric, Staffan Strömblad, and Tami L. von Schalscha. 1999. Integrin $\alpha_{V} \beta_{3}$ Promotes M21 Melanoma Growth in Human Skin by Regulating Tumor Cell Survival. Cancer Research 59:2724-2730.

Polato, Federica, Annamaria Codegoni, and Robert Fruscio. 2005. PRL3 Phosphatase Is Implicated in Ovarian Cancer Growth. Clinical Cancer Research 11:6835-6839.

Prewett, M, M Rothman, and H Waksal. 1998. Mouse-human chimeric anti-epidermal growth factor receptor antibody C225 inhibits the growth of human renal cell carcinoma xenografts in nude mice. Clinical Cancer Research 4:2957-2966.

Priestley, M.B. 1981. Spectral Analysis and Time Series. Academic Press.

Reinmuth, Niels, Fan Fan, Wenbiao Liu, Alexander A. Parikh, Oliver Stoeltzing, Young D. Jung, Corazon D. Bucana, Robert Radinsky, Gary E. Gallick, and Lee M. Ellis. 2002. Impact of Insulin-Like Growth Factor Receptor-I Function on Angiogenesis, Growth, and Metastasis of Colon Cancer. Laboratory Investigation 82:1377-1389.

Richmond, Ann, David H. Lawson, and Daniel W. Nixon. 1983. Extraction of a Melanoma Growth-stimulatory Activity from Culture Medium Conditioned by the Hs0294 Human Melanoma Cell Line. Cancer Research 43:2106-2112. 
Ricker, Justin L., Zhong Chen, and Xin Ping Yang. 2004. 2Methoxyestradiol Inhibits Hypoxia-Inducible Factor $1 \alpha$, Tumor Growth, and Angiogenesis and Augments Paclitaxel Efficacy in Head and Neck Squamous Cell Carcinoma. Clinical Cancer Research 10:8665-8673.

Robertson-Tessi, Mark, Ardith El-Kareh, and Alain Goriely. 2012. A mathematical model of tumor-autoimmune interactions. Journal of Theoretical Biology 294:56-73.

Roose, Tiina, S. Jonathan Chapman, and Philip K. Maini. 2007. Mathematical Models of Avascular Tumor Growth. Journal of Theoretical Biology 49:179-208.

Sanga, Sandeep, John P Sinek, Hermann B Frieboes, Mauro Ferrari, John P Fruehauf, and Vittorio Cristini. 2006. Mathematical modeling of cancer progression and response to chemotherapy. Expert Rev Anticancer Ther 6:1361-1376.

Sarraf, Pasha, Elisabetta Mueller, Daniel Jones, Frederick J. King, Daniel J. DeAngelo, Jeffrey B. Partridge, Sylvia A. Holden, Lan Bo Chen, Samuel Singer, Christopher Fletcher, and Bruce M. Spiegelman. 1998. Differentiation and reversal of malignant changes in colon cancer through PPAR $\gamma$. Nature Medicine 4:1046-1052.

Schirner, M, J Hoffmann, and A Menrad. 1998. Antiangiogenic chemotherapeutic agents: characterization in comparison to their tumor growth inhibition in human renal cell carcinoma models. Clinical Cancer Research 4:1331-1336.

Sharma, Sherven, Marina Stolina, Ying Lin, Brian Gardner, Patrice W. Miller, Mitchell Kronenberg, and Steven M. Dubinett. 1999. T CellDerived IL-10 Promotes Lung Cancer Growth by Suppressing Both T Cell and APC Function. Journal of Immunology 163:5020-5028.

Sheng, Hongmiao, Jinyi Shao, Susan C. Kirkland, Peter Isakson, Robert J. Coffey, Jason Morrow, R. Daniel Beauchamp, and Raymond N. DuBois. 1997. Inhibition of Human Colon Cancer Cell Growth by Selective Inhibition of Cyclooxygenase-2. The Journal of Clinical Investigation 99:2254-2259.

Shi, W, and DW Siemann. 2002. Inhibition of renal cell carcinoma angiogenesis and growth by antisense oligonucleotides targeting vascular endothelial growth factor. British Journal of Cancer 87:119-126. 
Slezak, Diego FernĞndez, Cecilia SuĞrez, Guillermo A. Cecchi, Guillermo Marshall, and Gustavo Stolovitzky. 2010. When the Optimal Is Not the Best: Parameter Estimation in Complex Biological Models. PLoS 5.

Smith, Matthew C. P., Kathryn E. Luker, and Joel R. Garbow. 2004. CXCR4 Regulates Growth of Both Primary and Metastatic Breast Cancer. Cancer Research 64:8604-8612.

Sunwoo, John B., Zhong Chen, and Gang Dong. 2001. Novel Proteasome Inhibitor PS-341 Inhibits Activation of Nuclear Factor- $\kappa$ B, Cell Survival, Tumor Growth, and Angiogenesis in Squamous Cell Carcinoma. Clinical Cancer Research 7:1419-1428.

Takahashi, Takashi, David Carbone, and Toshitada Takahashi. 1992. Wildtype but not Mutant p53 Suppresses the Growth of Human Lung Cancer Cells Bearing Multiple Genetic Lesions. Cancer Research 52:2340-2343.

Tan, Daniel S.-W., Marco Gerlinger, Bin-Tean Teh, and Charles Swanton. 2010. Anti-cancer drug resistance: Understanding the mechanisms through the use of integrative genomics and functional RNA interference. European Journal of Cancer 46:2166-2177.

Todaro, Matilde, Mileidys Perez Alea, Anna B. Di Stefano, Patrizia Cammareri, Louis Vermeulen, Flora Iovino, Claudio Tripodo, Antonio Russo, Gaspare Gulotta, Jan Paul Medema, and Giorgio Stassi. 2007. Colon Cancer Stem Cells Dictate Tumor Growth and Resist Cell Death by Production of Interleukin-4. Cell Stem Cell 1:389-402.

Tsubouchi, Yasunori, Hajime Sano, Yutaka Kawahito, Shigehiko Mukai, Ryoji Yamada, Masataka Kohno, Ken-ichiro Inoue, Timothy Hla, and Motoharu Kondo. 2000. Inhibition of Human Lung Cancer Cell Growth by the Peroxisome Proliferator-Activated Receptor- $\gamma$ Agonists through Induction of Apoptosis. Biochemical and Biophysical Research Communications 270:400-405.

Vogler, Meike, Henning Walczak, and Dominic Stadel. 2009. Targeting XIAP Bypasses Bcl-2 Mediated Resistance to TRAIL and Cooperates with TRAIL to Suppress Pancreatic Cancer Growth In vitro and In vivo. Cancer Research 68(19):7956-7965.

Warren, Robert S., Hui Yuan, Mary R. Matli, Nancy A. Gillett, and Napoleone Ferrara. 1995. Regulation by Vascular Endothelial Growth Fac- 
tor of Human Colon Cancer Tumorigenesis in a Mouse Model of Experimental Liver Metastasis. Journal of Clinical Investigation 95:1789-1797.

Willingham, Stephen B. et al. 2012. The CD47-signal regulatory protein alpha $(\operatorname{SIRP} \alpha)$ interaction is a therapeutic target for human solid tumors. PNAS 10.

Winkler, Gerhard. 2003. Image Analysis, Random Fields and Markov Chain Monte Carlo Methods. Springer-Verlag Berlin Heidelberg New York, 2nd ed.

Wong, Chun-Ming, Judy Wai-Ping Yam, and Yick-Pang Ching. 2005. Rho GTPase-Activating Protein Deleted in Liver Cancer Suppresses Cell Proliferation and Invasion in Hepatocellular Carcinoma. Cancer Research 65:8861-8868.

Yokoyama, Yumi, Mohanraj Dhanabal, and Arjan W. Griffioen. 2000. Synergy between Angiostatin and Endostatin: Inhibition of Ovarian Cancer Growth. Cancer Research 60:2190-2196.

Zender, Lars, Mona S. Spector, Wen Xue, Peer Flemming, Carlos CordonCardo, John Silke, Sheung-Tat Fan, John M. Luk, Michael Wigler, Gregory J. Hannon, David Mu, Robert Lucito, Scott Powers, and Scott W. Lowe. 2006. Identiin Acation and Validation of Oncogenes in Liver Cancer Using an Integrative Oncogenomic Approach. Cell 125:1253-1267.

Zender, Lars, Wen Xue, Johannes Zuber, Camile P. Semighini, Alexander Krasnitz, Beicong Ma, Peggy Zender, Stefan Kubicka, John M. Luk, Peter Schirmacher, W. Richard McCombie, Michael Wigler, James Hicks, Gregory J. Hannon, Scott Powers, and Scott W. Lowe. 2008. An Oncogenomics-Based In Vivo RNAi Screen Identifes Tumor Suppressors in Liver Cancer. Cell 135:852-864.

Zervos, Emmanuel E., James G. Norman, William R. Gower, Michael G. Franz, and Alexander S. Rosemurgy. 1997. Matrix Metalloproteinase Inhibition Attenuates Human Pancreatic Cancer Growth in Vitro and Decreases Mortality and Tumorigenesis in Vivo. Journal of Surgical Research 69(2):367-371.

Zhang, Ling, Xiaoyang Ren, Eckhard Alt, Xiaowen Bai, Shaoyi Huang, Zhengming Xu, Patrick M. Lynch, Mary P. Moyer, Xian-Feng Wen, and Xiangwei $\mathrm{Wu}$. 2010. Chemoprevention of colorectal cancer by targeting APC-deficient cells for apoptosis. Nature 464:1058-1063. 